\title{
Probing Molecular Stoichiometry by Photon \\ Antibunching and Nanofluidics Assisted Imaging in Solution
}

\author{
Dissertation \\ for the award of the degree \\ "Doctor rerum naturalium", \\ of the Georg-August-Universität Göttingen \\ within the doctoral program GGNB \\ of the Georg-August University School of Science (GAUSS) \\ submitted by \\ Hao Cheng \\ from Wuhan, China
}

Göttingen 2017 



\section{Thesis Committee:}

\section{Prof. Dr. Jörg Enderlein (Referee)}

Third Institute of Physics - Biophysics

Georg-August-University Göttingen

\section{Prof. Dr. Helmut Grubmüller}

Department of Theoretical and Computational Biophysics

Max Planck Institute for Biophysical Chemistry

Prof. Dr. Fred S. Wouters

Institute for Neuropathology

University Medical Center Göttingen

Other Members of the Examination Board:

\section{Dr. Andreas Neef (Co-Referee)}

Center for Biostructural Imaging of Neurodegeneration

University Medical Center Göttingen

Prof. Dr. Andreas Janshoff

Institute for Physical Chemistry

Georg-August-University Göttingen

Prof. Dr. Stefan Jakobs

Department of NanoBiophotonics

Max Planck Institute for Biophysical Chemistry

Date of oral examination: 18.05.2017 


\section{Affidavit}

Hereby, I declare that the presented thesis has been written independently and with no other sources and aids than quoted.

Göttingen, 22.03.2017 


\section{Contents}

\begin{tabular}{ll}
\hline Abstract & 1
\end{tabular}

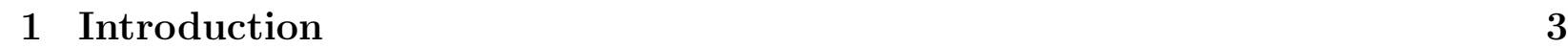

$1.1 \quad$ Probing molecular stoichiometry on the single-molecule level . . . . . . . . . 5

1.2 Photon antibunching . . . . . . . . . . . . . . . . . . . . 8

1.3 Brightness analysis $\ldots \ldots \ldots \ldots \ldots$

1.4 Nanofluidics assisted single-molecule detection . . . . . . . . . . . . . 13

2 Exploring photon antibunching to determine the stoichiometry of molec-

$\begin{array}{ll}\text { ular complexes } & 17\end{array}$

2.1 Theoretical background $\ldots \ldots \ldots \ldots \ldots \ldots$. . . . . . . . . . . . . . 17

2.2 Experimental: probing the stoichiometry of single-molecule and determining

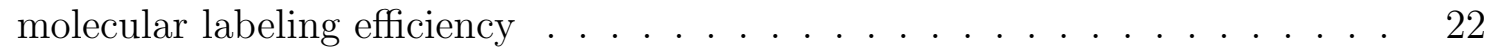

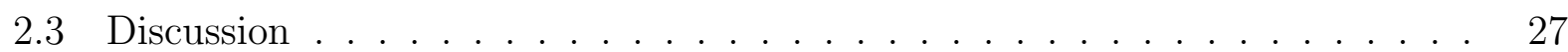

3 Single-molecule detection and manipulation in nanofluidic channels 29

3.1 Nanoconfinements in single-molecule detection . . . . . . . . . . . . . . . . . 29

$3.1 .1 \quad$ Nanocontainers . . . . . . . . . . . . . . . . . . . . . . . 30

3.1 .2 Micro/nanofabricated platforms . . . . . . . . . . . . . . . . 31

$3.2 \quad$ Single-molecule detection in 1D/2D Nanochannels $\ldots \ldots \ldots$. . . . . . . . 35

3.2 .1 Confocal-based detection $\ldots \ldots \ldots \ldots$

3.2 .2 Imaging-based detection $\ldots \ldots \ldots \ldots$. . . . . . . . . . 37

3.3 Single-molecule manipulation in 1D Nanochannels . . . . . . . . . . . . . . . 42

$3.3 .1 \quad$ Electrokinetic flow $\ldots \ldots \ldots$. . . . . . . . . . . . . . . . . . . 42

3.3 .2 Single-molecule recycling and trapping . . . . . . . . . . . . . . . 44

4 Imaging-based photon counting histogram (iPCH) for measuring the distribution of molecular mixtures $\quad 49$

4.1 Stroboscopic imaging . . . . . . . . . . . . . . . . . . . . . 50

4.2 Precise brightness characterization $\ldots \ldots \ldots \ldots$

4.2 .1 Shot noise $\ldots \ldots \ldots \ldots \ldots \ldots$ 
4.2 .2 Background . . . . . . . . . . . . . . . . . . . . . . 57

$4.2 .3 \quad$ EMCCD effect $\ldots \ldots \ldots \ldots \ldots \ldots$

4.2 .4 Excitation profile $\ldots \ldots \ldots \ldots \ldots$

4.2 .5 Photophysics . . . . . . . . . . . . . . . . . 60

4.2 .6 Single fluorophore brightness distribution . . . . . . . . . . . . . 63

4.3 Applying iPCH to reveal the distribution of molecular mixtures . . . . . . . 64

4.3 .1 Disentangling the mixtures of two dyes . . . . . . . . . . . . . 64

4.3 .2 DNA sizing . . . . . . . . . . . . . . . . . . . . . 66

$4.3 .3 \quad$ Determining distributions of stoichiometries for molecular mixtures . 68

$4.4 \quad$ Estimating diffusion coefficient by spot size analysis . . . . . . . . . . . . . . 70

$\begin{array}{lll}5 & \text { Conclusions and outlook } & 75\end{array}$

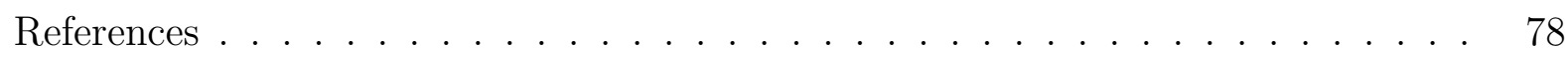

Acronyms . . . . . . . . . . . . . . . . . . . . . . . . 92

Acknowledgments . . . . . . . . . . . . . . . . . . . . 93 


\section{Abstract}

A mechanistic understanding of biological function requires a quantitative determination of macromolecular subunit architecture and interaction. Optical microscopy and spectroscopy provide a noninvasive method to characterize the stoichiometric ratios of molecular complexes. Though target-bound fluorescence labeling techniques can help to detect single molecules, counting molecules in a molecular complex remains challenging. In solution, diffusion limits the observation times of single molecules and, thus reduces the number of detectable photons. Current methods have limited resolving power or are constrained by a complex experimental configuration. Therefore, they are not able to precisely quantify the number of labeled fluorophores. In this dissertation, I first explore the ability of photon antibunching to probe molecular stoichiometry in solution. The underlying theoretical model is elucidated and subsequently applied to samples of different labeling stoichiometry. It enables determining the average number of emitters per molecular complex. In the second part of my thesis, to obtain the full distribution of species with a particular number of fluorescent labels, another method is developed. It is based on molecular brightness analysis using imaging-based photon counting histograms. This is assisted by a nanofluidic device that enables direct imaging of diffusing molecules with extended observation time. I performed a systematic study of the experimental conditions which guarantee an optimal performance of this method. The capability of correctly determining distributions of stoichiometries of molecular mixtures is verified by both simulation and measurements of small molecules. The nanofluidics system allows both single-molecule detection and manipulation under microscopic imaging, which is simple and implementation-friendly. 


\section{Introduction}

In the early 1990s, adventurous biophysicists and microscopists invented an optical system to eventually reach the detection of single molecules 84 . 88 . Aided by fluorescent tagging, e.g. chemical labeling, immunofluorescent staining, and genetic labeling 101 that can specifically target the molecule/structure of interest, this groundbreaking advance allows for seeing single biomolecules as brightly shining stars dancing in their biological space. Since then, singlemolecule detection, with its potential to gain invaluable insights into nature, has inspired

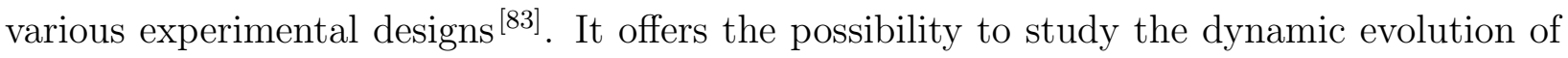
biological systems at unprecedented molecular scale. Especially, single-molecule fluorescence localization-based microscopy has not only allowed for sensing of minuscule molecule mobility, e.g. motor protein walking along actin filaments ${ }^{124}$ but also contributed essentially to break the longstanding diffraction barrier of optical microscopy 99 . 99 . It has turned the optical microscope into the nanoscope and created a vibrant cross-disciplinary research environment for super-resolution imaging ${ }^{89}$. For wide-field epifluorescence or total internal reflection fluorescence (TIRF) microscopes, the resolution of imaging can be increased by at least one order of magnitude. This family of methods takes advantage of the feature that fluorescence molecules can be switched 'on' and 'off'. Therefore, each 'on' molecules can be recorded and separately localized in a time-sequential manner ${ }^{100}$.

Super-resolution microscopy tells the position of biomolecules with improved precision, which is a very useful tool such as to directly observe if binding and dissociation take place for an enzyme to its substrate $\underset{52}{ }$. To report on the intramolecular conformational changes or on intermolecular interactions between molecules, another powerful technique, named singlemolecule Foerster Resonance Energy Transfer (smFRET), is used. It normally measures the fluorescence time trajectories from a FRET pairs, typically two adjacent fluorophores of different colors called donor and acceptor. Dipole-dipole interaction between the two fluorophores results in energy transfer from donor to acceptor that is a sensitive function of distance. Using SmFRET, one can measure molecular distances and their temporal variation in a distance range of 2 to $10 \mathrm{~nm}[51,[9][106]$.

Although single-molecule methods were instrumental in providing previously unavailable data on elementary biological processes ${ }^{[50}$, many important technical challenges remain to 
be solved. Among them, two issues have frequently been raised in the context of biological applications and call for improvement: immobilization-free single-molecule detection method to avoid the perturbation of biological functions of studied molecules ${ }^{[5}$ and obtaining quantitative information, in particular, counting molecule numbers in biomolecular complexes 100$]$. Nanometer-sized objects are undergoing fast Brownian motion in aqueous solution, resulting in the difficulty of studying non-immobilized biomolecules. Optical detection of molecules in solution is often made difficult by the out-of-focus fluorescence background from diffusing molecules. The most natural solution is to introduce some forms of optical-sectioning ${ }^{5}$, which can highlight only a fraction of molecules in the sample volume by confined excitation/detection. For example, confocal spectroscopy is commonly applied to detect single molecules in solution. The light beam is tightly focused by using a high numerical aperture (NA) objective, producing a diffraction-limited excitation volume. A spatial pinhole placed at the confocal plane of the objective allows for eliminating out-of-focus light (optical sectioning). Only molecules diffusing through the confocal detection volume can emit fluorescence signal that is recorded onto point detectors of single photon sensitivity ${ }^{5}$. Every molecular detection event appears in the fluorescence intensity time trace as a transient signal burst 106. Burst-by-burst analysis can be performed to extract information of each diffusing molecules. One of the drawbacks is that the dwell time of a single molecule in the confocal detection volume ( $\sim 1$ femtoliter) is limited by diffusion so that each molecule can only be observed for a short moment (typically, a few milliseconds). Concerning biological applications, many processes happen on the timescale of seconds. Thus, the milliseconds observation time is too short to follow the dynamics of slow process 117 . Likewise, for imaging method such as TIRF microscopy, laser illumination is performed with an incidence angle above the critical angle of total internal reflection. It generates an evanescent excitation field that forms a thin optical excitation layer of a few hundred nanometers in thickness 4 . Diffusing molecules quickly escape from this detection plane, which also limits the number of photons that can be collected (i.e. photon budget) during the short observation time. The photon budget determines the accuracy with which one can determine single molecule properties, for example how well one can localize a single molecule from its image. As is well known, this localization accuracy scales with the square root of the number of detected photons. A similar relation will apply when one wants to determine the number of fluores- 
cently emitting molecules on a molecular complex by measuring the brightness (number of photons per time) from such a complex.

This dissertation focuses on determining the stoichiometry of biomolecular complexes in solution on the single-molecule level. For this purpose, I have developed a modified fluorescence photon antibunching method, and I have built a new nanofluidics-based rapid single-molecule imaging microscope. Using this microscope, I was able to determine singlemolecule brightness histograms, which can be used to obtain distributions of stoichiometries across an ensemble of molecules.

\subsection{Probing molecular stoichiometry on the single-molecule level}

Single-molecule spectroscopy and imaging are able to measure the full distribution of molecular parameters (e.g. emission spectra, brightness or fluorescence lifetime) instead of only a population average. It helps to explore the hidden heterogeneities of molecular properties, such as the presence of multiple subpopulations, or the presence of observe intermediate states in molecular dynamics 75 .

Single-molecule experiments have been performed both in vivo and in vitro. The two approaches are complementary and used in combination to study molecular functions in cells. In vivo measurements can reveal how biomolecules (such as proteins or DNA) function in a cellular context, and the actions of individual molecules are connected to the phenotype of a cell ${ }^{95}$. For in vitro measurements, molecules are usually separated spatially at low density, and their activity can be determined with high precision. Additionally, in vitro experimental results are often used as prior expectations to the related in vivo experiments.

Determining molecular stoichiometry is of great significance. Knowledge of subunit stoichiometry is essential to infer the biological functions of molecular complexes in cells $[57$. For instance, many membrane proteins form multimers before they achieve a functional states and the stoichiometry of molecular assemblies determines the fundamental properties of signaling complexes 116]. Moreover, altered stoichiometries may lead to disease states of the cell [96. Thus, quantitative information is helpful for better understanding the underlying molecular mechanisms of many biological problems. 
For example, neurodegenerative diseases such as Alzheimer's disease (AD), Parkinson's disease $(\overline{P D})$, Huntington's disease (HD) or amyotrophic lateral sclerosis (ALS) have a common molecular mechanism, specifically, the aggregation of misfolded proteins in the central nervous system [97 [8]. More than half of the 25 recognized neurodegenerative diseases are associated with protein aggregates $[90$. However, a quantitative characterization of protein aggregation is challenging ${ }^{[58}$, and the fundamental reactions taking place during aggregation are difficult to determine 104 . Also, the exact pathways and kinetics of protein aggregation are not well known, although the peculiarities of aggregation are probably important for disease development. $\frac{98}{}$.

Recently, single-molecule spectroscopy has been applied to study PD-associated protein alpha-synuclein $(\alpha S)$ oligomerization ${ }^{26}$. More specifically, smFRET was employed to detect and characterize oligomer structures. This measurement technique is capable of detecting the crucial early stages of aggregation and has eventually revealed the presence of two forms of oligomeric species ${ }^{[26}$. A recent combination of fast-flow microfluidics and kinetic analysis has been used to drastically increase the temporal resolution of oligomerization measruements $[56]$ [58].

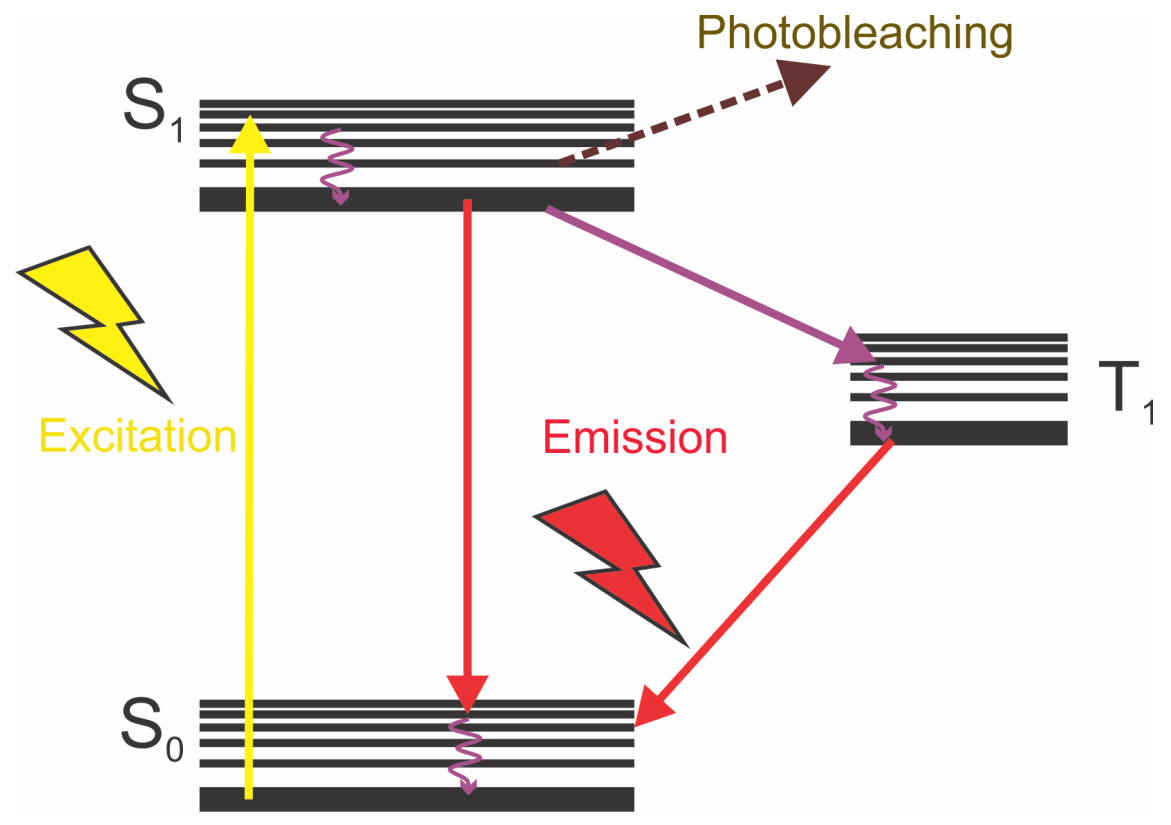

Figure 1: Jablonski diagram of the energy levels

Single molecule spectroscopy and imaging can be particularly useful for this kind of studies. 
Super-resolution microscopy offers the opportunity to directly image individual protein aggregates. For instance, direct stochastic optical reconstruction microscopy (dSTORM) has been used to image the arrangement and stoichiometry of proteins of the nuclear pore complex, with $\sim 15 \mathrm{~nm}$ lateral resolution. It was shown that the integral membrane protein gp210 is distributed in an eightfold radial symmetry ${ }^{76}$. Nonetheless, even super-resolution imaging is not yet capable of spatially resolving densely packed molecular aggregates. Fortunately, single-molecule fluorescence spectroscopy can provide information about the stoichiometry of such clusters or complexes.

As shown in Figure 1, a single fluorescent molecule absorbs a photon from the excitation source and is excited from its electronic singlet ground state $S_{0}$ into its first singlet excited state $S_{1}$. After fast internal relaxation that brings the molecule to the lowest vibrational level of $S_{1}$, it emits a photon that is red-shifted with respect to the excitation wavelength and returns to its ground state. This transition from $S_{1}$ to $S_{0}$ is named spontaneous emission, and the average time a molecule resides in its excited state is called fluorescence lifetime. The excited-state lifetime (typically a few nanoseconds) determines the temporal separation between subsequent photons from a single emitter. Thus, a single emitter can only emit one photon at a time, which leads to the phenomenon of antibunching 65 64 [6]. However, when measuring two or more independent emitters, it is possible to detect simultaneously emitted photons. Therefore, anitbunching can be used to determine the stoichiometry of multimolecular complexes, both on surfaces ${ }^{[2]}$ and in solution ${ }^{109}$. The first part of this dissertation focuses on photon antibunching in solution and its application to probe molecular stoichiometry.

There exists a second approach to use single-molecule fluorescence for determining the number of emitting fluorophores on a single complex. After a certain number of excitation cycles, a fluorophore molecule will eventually photobleach. Consequently, the fluorescence intensity of a molecular complex with more than one label should drop in a stepwise fashion. The number of these discrete steps ideally corresponds to the number of fluorescent tags on one single complex. Hence, the method of counting bleaching steps has been combined with single-molecule localization-based microscopy for determining stoichiometry of immobilized molecular complexes [116 [128] 33. Besides photobleaching, many fluorophores can reversibly switch between a fluorescence 'on' and a dark 'off' state, due to triplet or 
redox state dynamics, photochromic switching ${ }^{[52]}$ or dynamic binding and dissociation $[60]$. Recent studies have explored fluorophore blinking statistics to count molecules in molecular complexes 68 [96 59 57. Using photoleaching and photoblinking to determine molecular stoichiometry usually requires long time observations of single molecules, either to ensure the capture of intensity drops or to record many on/off cycles for statistical estimation. Therefore, they are mostly working on immobilized molecules.

One goal of the dissertation is to study molecules in aqueous solution. In this case, the biggest challenge is the short dwell time for observing a single diffusing molecule, i.e. the short time a molecule resides within the detection volume before diffusing out of it. For these measurements, fluorescence blinking and bleaching are undesired. Molecular diffusion, both rotational and translational, is connected to the hydrodynamic size of a molecule. Fluorescence Correlation Spectroscopy $(\underline{\mathrm{FCS}})$ is typically used for measuring the hydrodynamic radius of molecules at nanomolar concentrations, which could potentially be used for determining the stoichiometry of a complex [1] 28. However, this technique is less suitable for the measurements of a mixture of multiple species, especially when the size difference is not so significant. Alternatively, molecular brightness, which is defined as the average number of photons per time per molecule, provides a sensitive parameter for measuring the distribution of molecular species with different stoichiometric numbers. Provided that there are no electronic interactions between the fluorophores on one complex, brightness of a molecular complex scales linearly with the number of fluorescent emitters. Thus, the second part of this dissertation is devoted to the precise measurement of molecular brightness and its application to the determination of stoichiometries in mixtures.

\subsection{Photon antibunching}

Photon antibunching was first theoretically predicted [13] [65] and then experimentally demonstrated in the late $1970 \mathrm{~s}^{[64}$, when resonance fluorescence of sodium atoms was studied under continuous excitation in atomic beams. Shortly after, Moerner and Orrit accomplished the detection of single molecules at low temperature ${ }^{84}$, 88 , and photon antibunching was successfully measured for a single dye molecule trapped in a solid ${ }^{6}$. It was later observed for 
organic dyes on a surface ${ }^{3]}$, for semiconductor nanocrystals ${ }^{81}$ and for nitrogen-vacancies in diamond $[\sqrt[66]{6}$ at room temperature. Since then, photon antibunching has been routinely applied as the gold standard of proving the presence of only a single emitter within the detection volume $\underline{47}$.

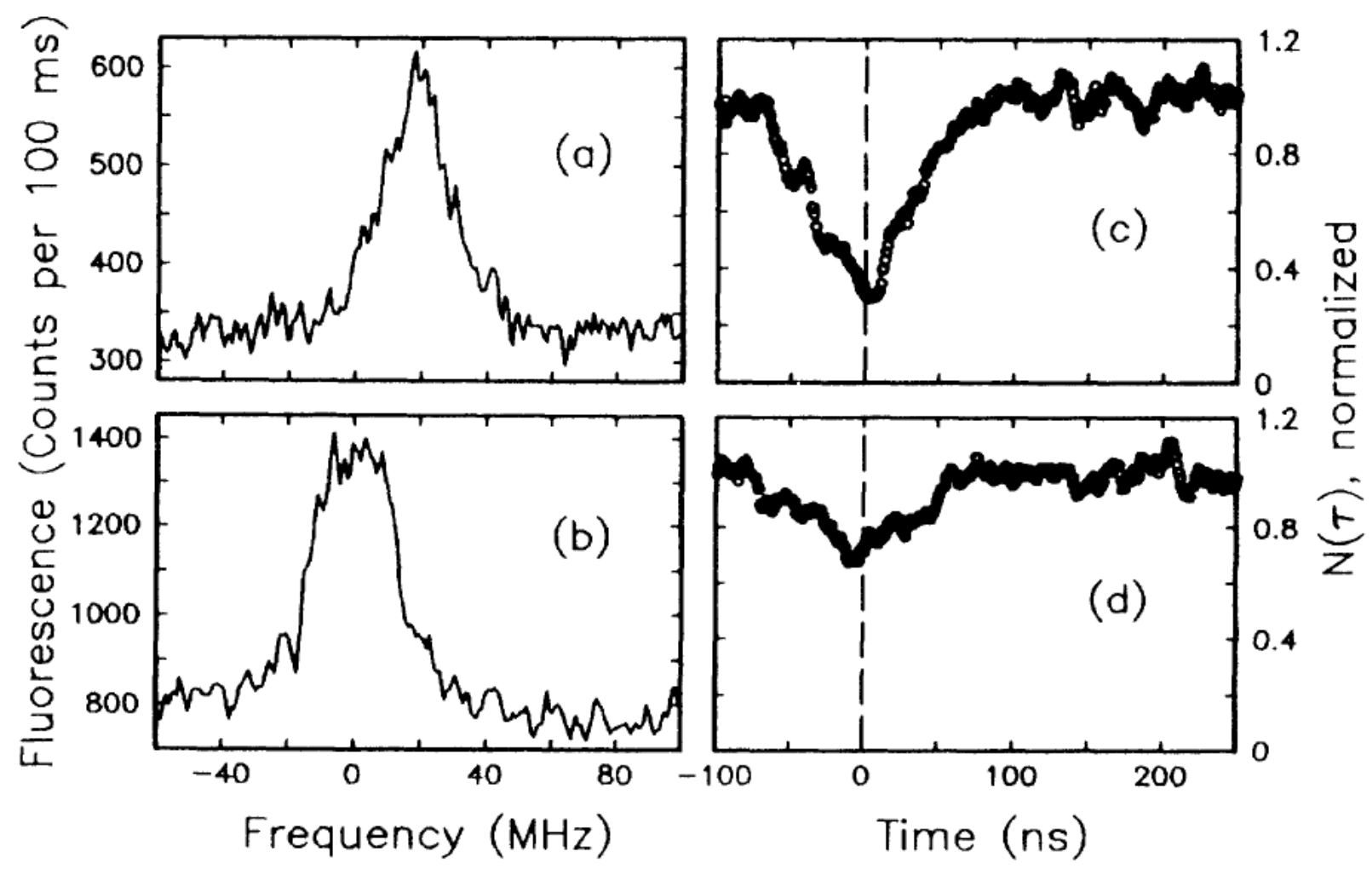

Figure 2: Fluorescence excitation spectra of (a) one and (b) two single pentacene molecules. (c) and (d) show correlation function for cases (a) and (b), from ref. ${ }^{6}$. Note that the contrast in the correlation function in $(d)$ is much more reduced than $(c)$.

Modern photon counting devices achieve a temporal resolution of as small as 1 ps, which is enough for observing fluorescence photon antibunching that typically takes place on a time scale of nanoseconds. The measurement is done by counting single photons with high temporal resolution and then calculating a two-photon correlation function. Antibunching will be visible as a sharp drop of the two-photon correlation amplitude at short lag times on the order of the fluorescence lifetime. This is due to the fact that a single molecule cannot emit, on average, more than one photon during the lifetime of its excited state. More specifically, no photon pairs can be detected simultaneously, yielding a zero value at zero lag 
time of the correlation function. It is obvious that this value increases when the lag time becomes larger than the fluorescence lifetime. If more than one emitter is present within the detection volume, those emitters can emit photons independently. This results in a non-zero probability of detecting two photons at zero lag time, and the amplitude of the antibunching dip becomes smaller with an increasing number of emitters. In the earliest single-molecule experiments of photon antibunching, this unique feature has already been observed for the case of two molecules trapped in a solid and pumped at the same time ${ }^{6}$, as shown in Figure 2 .

Thus, photon antibunching can be used as a tool to determine the number of emitters within the detection volume. Given that molecules in solution are indistinguishable, photon antibunching measurements can be performed in a cumulative way by integrating the fluorescence signal over many single-molecule transits through the detection volume.

I will discuss the method of using photon antibunching for the determination of molecular stoichiometry in Chapter 2. I will first present the theoretical background of the method, and then present the results of my experimental application of antibunching to determining molecular stoichiometries.

\subsection{Brightness analysis}

Fluorescence properties such as intensity or lifetime can be used to sense local changes in a molecule's environment. In order to guarantee a reliable statistical analysis, a sufficiently large number of detected photons is required. For example, fluorescence lifetime is a sensitive indicator that can be applied to investigate the dynamics of single molecules, e.g. the protein conformational changes ${ }^{123}$. For reliably determining the fluorescence lifetime of a mono-exponential fluorescence decay, one needs at least 100 detected photons ${ }^{80}$. Many more photons are required for multi-exponential decays. To allow for long observation times, measurements are typically done by performing point-like excitation/detection of individual tethered molecules. In solution, the observation time of each molecule is limited due to its Brownian diffusion through the detection volume, usually in the range of less than $1 \mathrm{~ms}$ for a GFP-like protein. Under a typical molecular count rate in the order of $10^{4}$ to $10^{5}$ counts $/ \mathrm{s}$, as a consequence, it is difficult to record enough photons to give a reliable estimation of 
single molecule lifetime.

However, freely diffusing molecules in solution experience a more homogeneous local environment than immobilized molecules. Given that the independently fluorescing molecules within a molecular complex share the same emission properties, it is possible to increase the statistics by accumulating the signal over many molecular detection events.

A confocal microscope provides an exceptional signal to noise ratio (SNR), in comparison to a wide-field fluorescence microscope. There, each molecule passing through the detection volume is excited by light of a tightly focused laser beam. If the concentration of fluorescing molecules in solution is low enough, the recorded fluorescence intensity will show large fluctuations over time, due to the constantly changing number of fluorescing molecules within the detection volume. Early work concerned with statistical analysis of this signal fluctuation has resulted in techniques such as photon-counting histogram ( $(\underline{\mathrm{PCH}})$ spectroscopy 15 16 , or Fluorescence Intensity Distribution Analysis (FIDA ${ }^{62}$. The methods employ different mathematical approaches to determine the molecular brightness and number of molecules in the confocal detection volume. Both techniques focus on investigating the fluctuations of the fluorescence signal amplitude, which is mainly caused by the random number of molecules in the detection volume, and the non-uniform spatial profile of the excitation intensity. Experimental conditions should have to be carefully characterized to fit the data with a sophisticated theoretical model. In particular, precise determination of the so-called molecular detection function (MDF) is required. The MDF depends on excitation and detection light geometry and emission properties of the molecule, and it tends to change by multiple experimental parameters such as cover-slide thickness, refractive index of sample solution, astigmatism and optical saturation ${ }^{36}$. Thus, those methods of brightness analysis have not found broad biological applications.

A fundamental challenge of brightness quantification in solution is that each diffusing molecule transits the $\sim 1$ fL detection volume along an irregular trajectory, which generates heterogeneous fluorescent fluctuation time traces. Pioneered by Keller and his group, the idea of flow cytometry has been introduced in combination with single-molecule burst size distribution (BSD) analysis 79 [37 [38. In their study, a sheath flow directed and transported single molecules through a laser focus, ensuring nearly identical molecular transit trajectories. This generated relatively uniform fluorescence bursts, as demonstrated in Figure 3. 
Even though excellent signal quality and unsurpassed resolving power for molecular mixtures were achieved at that time, this method has not been applied widely. This is due to the complexity of the set-up that requires an orthogonal excitation/detection scheme which has to be well-aligned to the sheath flow. Moreover, since it allows the presence of only one molecule at one time in the detection volume, the experimental throughput is very low. A follow-up method of Cylindrical Illumination Confocal Spectroscopy (CICS) ${ }^{74}$ utilizes one-dimensional beam shaping to create a uniform sheet-like observation volume that could be incorporated into a versatile microfluidics system. It facilitates the application of Keller's BSD analysis, especially on many single nucleic acids related studies 72$]$ [73.

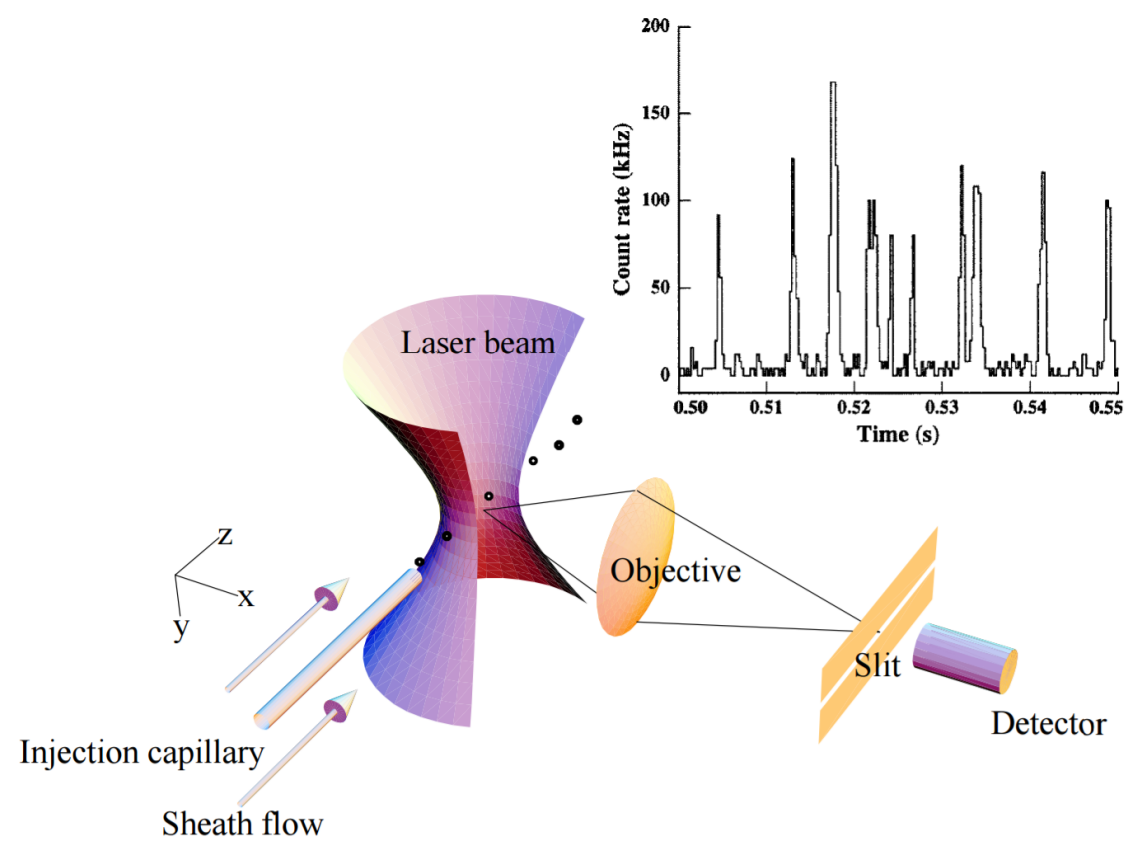

Figure 3: Single-molecule burst size analysis by flow cytometry. Modified from ref. [37] [38].

For all flow cytometric approaches to single-molecule detection, a relatively high flow rate (a few millimeters per second) is required to compete against molecular diffusion and, thus to ensure identical molecular transit paths. For a typical confocal-based detection volume of less than $1 \mu \mathrm{m}$ in diameter, the observation time (a few hundreds of $\mu \mathrm{s}$ ) becomes too short for detecting a sufficient number of fluorescent photons per molecule.

Alternatively, single-molecule studies can also be performed with an imaging widefield microscope. Generally, epifluorescence microscopy is applied to excite a large area of a sample, and 
multiple molecules can be recorded and studied in parallel. It can be integrated into a detection system for high-throughput applications. Additionally, since the individual molecules are visualized directly by imaging, locations of molecular detection can be used to infer the excitation condition that each molecule has been experiencing. This approach can significantly improve the brightness characterization of single moleucles, as discussed in Chapter 4. To extend the observation time and increase the photon budget, possible solutions include either devising a dynamic detection system that is able to track fast moving molecules or trapping them locally in the detection volume. Early work along these ideas has lead to elegant engineering solutions, e.g. single-molecule tracking [53] or Anti-Brownian Electrokinetic (ABEL) trapping ${ }^{22]}$. In a imaging method, long time observation may benefit from expanding illumination but would have to suffer from low SNR. Concerning the brightness analysis, it is of great interests that a simple integration of nanofluidic system offers to solve this problem in a straightforward manner. The characterization of molecular brightness is inherited from Keller's early work. In contrast, rather than illustrating the single-molecule detection event as fluorescence intensity burst, imaging system allows for visualizing single molecule as characteristic image pattern, i.e. Point Spread Function ( $\mathrm{PSF}$ ). Starting with Chapter 3, I will demonstrate how a nanofludic system allows for observing single molecules over long time spans, orders of magnitudes longer than what is usually possible with a confocal microscope, and how to measure their brightness. The imaging system substantially improves the throughput, which is important for application in e.g. advanced molecular diagnostics.

\subsection{Nanofluidics assisted single-molecule detection}

The combination of micro/nanofabrication techniques with wide-field optical microscopy is perfectly suited to achieve extended observation times for immobilization-free singlemolecule studies. With much less sample consumption and higher speed of analysis, micro/nanofluidics facilitates analytical biochemistry with chip-like devices 127 . These systems are especially efficient when utilizing optical signal transduction. Nanofluidic devices help in confining molecular motion to a few dozen or hundred nanometers. Therefore, fluorescence 
molecules can be kept in the detection volume. This offers an extended observation times which are not obtainable in free solution. Furthermore, this local confinement enables the separation of individual molecules and realizes some kind of physical sectioning similar to optical sectioning in confocal spectroscopy or TIRF microscopy, which provides a high SNR by largely reducing the out-of-focus background.

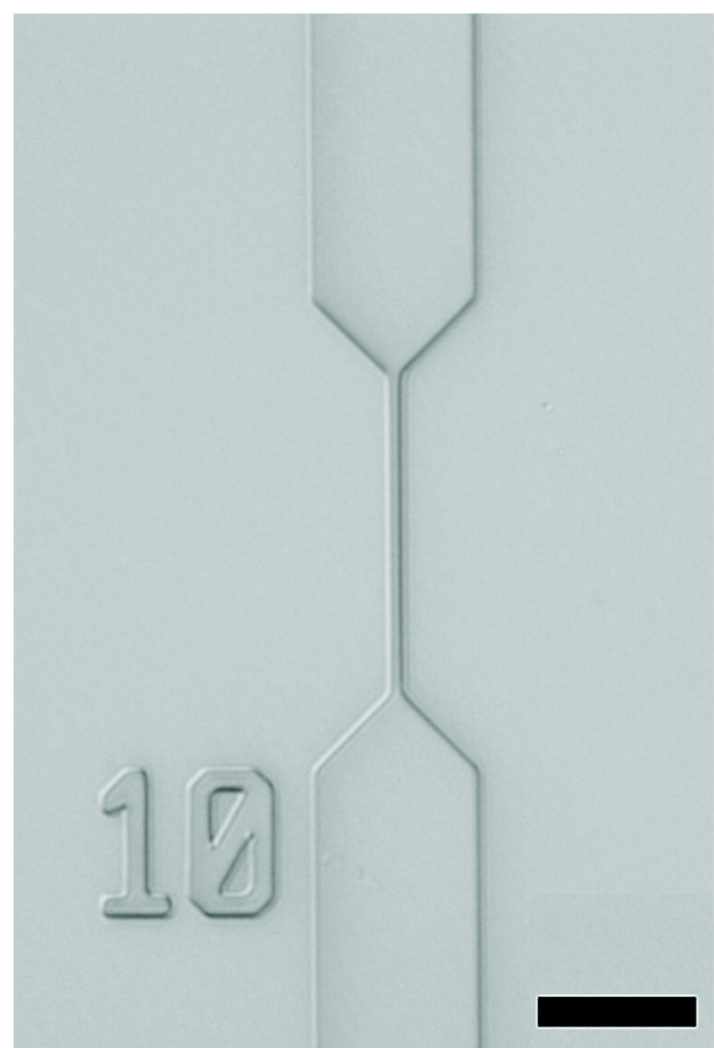

A

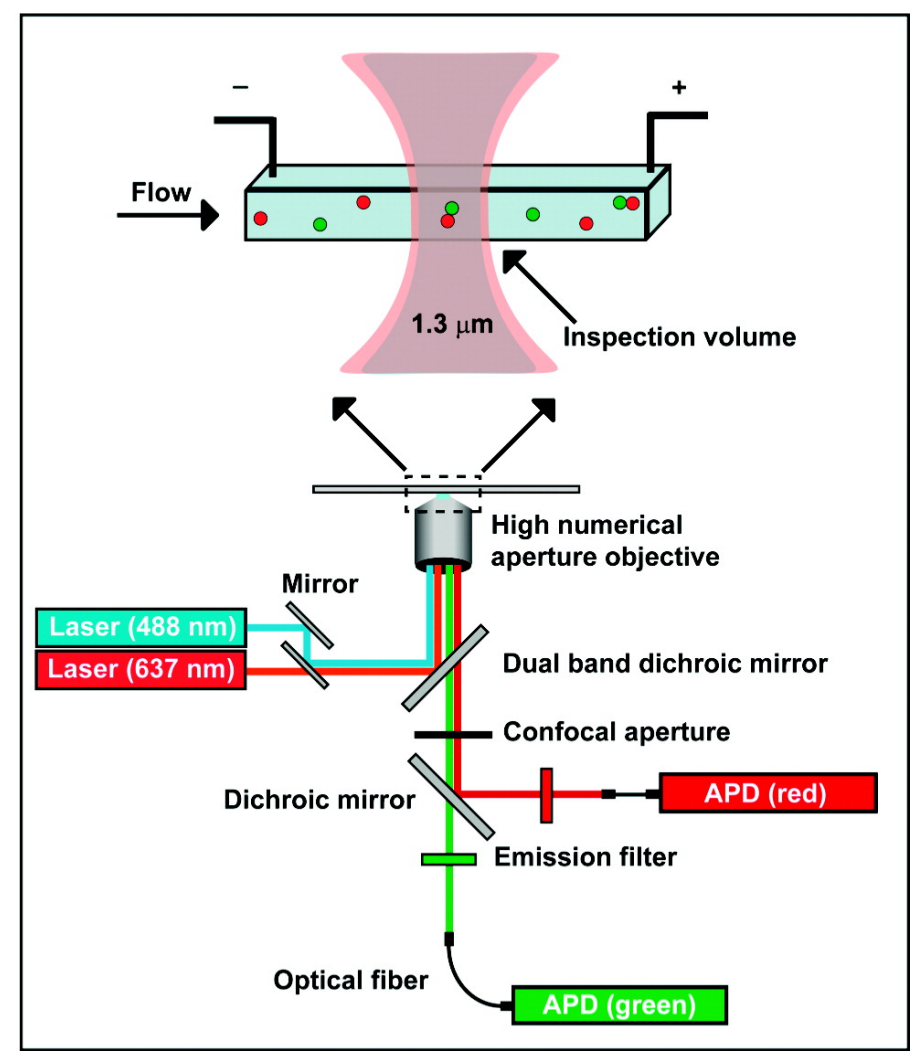

B

Figure 4: Nanofluidic channel system for single-molecule optical measurements. (A) A typical nanofluidic channel (500 $\mathrm{nm} \times 250 \mathrm{~nm}$ cross section) by differential interference contrast optical microscopy. The scale bar is $10 \mu \mathrm{m}$. (B) Schematic diagram of single-molecule detection in the nanofluidic channel under a confocal fluorescence microscope. Adapted from ref. [19].

One of the most prominent applications of this kind of technique is single molecule realtime sequencing (SMRT). It utilizes zero-mode waveguides ( (ZMWS $)$ to form thousands of zeptoliter small reaction vessels on a planar surface. Single nucleotide incorporations by DNA polymerase are observed through a massively parallel fluorescence detection 71 [35. Besides ZMWs, nano-sized fluidic channels are another way of confining molecules into well- 
determined excitation and detection geometries.

As shown in Figure 4, a nanofluidic system provides a flow cytometry-like device that is able to guide molecules through the optical detection volume. With the height of the channel smaller than the depth of focus, molecules which are confined within the channels are consistently kept in the focal plane. Therefore, Keller's early idea of single-molecule BSD analysis can be revived in a new fashion. The nanofluidic device is compatible to inverted fluorescence microscope, making the alignment of sample flow much easier.

Nanofluidic channels also enable long-term single-molecule investigation in solution by manipulating molecular motion ${ }^{23}$ [0]. ${ }^{20 m F R E T}{ }^{[15]}$ or lifetime spectroscopy ${ }^{118}$ in solution have already been successfully performed using nanoscale devices. It also offers the possibility to measure the distribution molecular brightness with high precision. Moreover, employing a single photon sensitive camera, brightness information can be obtained by direct imagingbased photon counting. This method can analyze multiple single molecules at a time and is amenable to massively parallel integration. Since the diffusion and transport of individual molecules are directly observed in nanofluidic channels, single-molecule brightness estimations can be done in a way that is no longer sensitive to the irregularity of molecular motion. For this purpose, full-glass-chips with channel heights of less than $200 \mathrm{~nm}$ are utilized, which confine molecular motion to the focal plane of the observing microscope. In combination with high-speed stroboscopic imaging and active flow control, individual molecules can be precisely monitored and well-manipulated in solution. I have obtained high-quality images of freely diffusing molecules and measured their brightness with high accuracy. The direct imaging approach is high-throughput and implementation-friendly, which offers great potential for many lab-on-a-chip based 'point-of-care' applications with single-molecule sensitivity 103$]$. 


\section{Exploring photon antibunching to determine the sto- ichiometry of molecular complexes}

Several studies have been published that have applied fluorescence antibunching for the determination of the number of independent emitters in immobilized molecular complexes [6] [119] [42], or to study energy transfer in fluorescently tagged biomolecules [27 113. In solution, the photophysical properties of fluorescent molecules are not influenced by any interaction with surfaces or polymers, as is typically the case for immobilized molecules. Thus, provided perfect control of excitation and detection conditions, each molecule in solution will show identical emission properties. Moreover, in solution one can achieve extremely high data quality by accumulating the fluorescence signal over many (thousands to millions) transits of single molecules through the detection volume. This is particularly important for fluorescence antibunching measurements, which require extremely high photon statistics, and thus for using antibunching for determining molecular stoichiometries. In this Chapter, I will start with introducing correlation functions and photon antibunching under continuous wave (CW) excitation and then move to the more efficient pulsed excitation mode. Furthermore, the influence of dye photophysics on antibunching measurements will be discussed. Finally, I will present experiments which validate the applicability of antibunching for determining the average stoichiometry of freely diffusing multi-emitter complexes.

\subsection{Theoretical background}

Typically, photon antibunching is measured by a confocal microscope with the single photon sensitive detector, which records the fluorescence signal as intensity time trace. The signal $I\left(t_{0}\right)$ contains photons from the fluorescence of molecules within the sample and the background $I_{b g}$, and reads:

$$
I\left(t_{0}\right)=\sum_{j} I_{j}\left(t_{0}\right)+I_{b g}\left(t_{0}\right)
$$

Here the summation effects to all $j$ molecules. The latter term is the background signal resulting from light scattering, electronic and detector noise. 
In FCS, fluorescence intensity time trace is correlated with a time-shifted replica of itself for different values of the time shift (i.e. lag time). The result is the Autocorrelation Function (ACF) and is written as:

$$
\begin{aligned}
g(t) & =\left\langle I\left(t_{0}\right) \cdot I\left(t_{0}+t\right)\right\rangle \\
& =\left\langle\left[\sum_{j} I_{j}\left(t_{0}\right)+I_{b g}\left(t_{0}\right)\right] \cdot\left[\sum_{k} I_{k}\left(t_{0}+t\right)+I_{b g}\left(t_{0}+t\right)\right]\right\rangle \\
& =\sum_{j}\left\langle I_{j}\left(t_{0}\right) \cdot I\left(t_{0}+t\right)\right\rangle+{\overline{I_{b g}}}^{2}+\sum_{j} \bar{I}_{j} \overline{I_{b g}}+\sum_{k} \overline{I_{k}} \overline{I_{b g}}+\sum_{j \neq k} \bar{I}_{j} \bar{I}_{k}
\end{aligned}
$$

Here the bars and triangular brackets denote the average over all time values $t_{0}$. Provided that no intermolecular interaction is present, photon emissions from different molecules are uncorrelated. Meanwhile, as all molecules in solution are indistinguishable and are treated equally, Eq. 2 is then reduced to calculate the ACF of a single molecule, which is given by:

$$
g_{1}(t)=\left\langle i\left(t_{0}\right) \cdot i\left(t_{0}+t\right)\right\rangle
$$

Here $i\left(t_{0}\right)$ denotes the recorded fluorescence signal from any individual molecule. Eq. 2 can be further written as:

$$
g(t)=N\left\langle i\left(t_{0}\right) \cdot i\left(t_{0}+t\right)\right\rangle+{\overline{I_{b g}}}^{2}+2 \cdot N\left\langle i\left(t_{0}\right)\right\rangle \overline{I_{b g}}+N(N-1)\left\langle i\left(t_{0}\right)\right\rangle^{2}
$$

where $N$ is the total number of molecules recorded. Using the sample volume $V$, one can write this equation in the form of molecular concentration $c=N / V$ and $N(N-1) / V^{2} \approx c^{2}$. There is

$$
g(t)=c g_{1}(t)+(c \bar{f}+b)^{2}
$$

Here, $\bar{f}$ is the average fluorescence of individual molecules and $b$ is the average detection background.

The fluorescence ACF describes the probability to detect a photon after lag time $t$ given that there was a photon detection event at time zero. The average photon counting rate from a single emitter (note the difference from single-molecule) at position $\mathbf{r}$ is described as $f_{s}(\mathbf{r})$. To the perspective of a single-molecule that contains the same independent fluorescent emitters of number $n$, the probability of detecting a photon at any time is then given as $n f_{s}(\mathbf{r})$.

Considering that a photon pair can either come from the same of the $n$ emitters or from two 
different emitters, on the timescale fast enough that it can regard the diffusing molecules as stationary, the single-molecule ACF will have the form:

$$
g_{1}=\left\langle\left\langle n f\left(\mathbf{r}, t_{0}+t\right) f_{s}\left(\mathbf{r}, t_{0}\right)+n(n-1) f_{s}\left(\mathbf{r}, t_{0}+t\right) f_{s}\left(\mathbf{r}, t_{0}\right)\right\rangle_{t_{0}}\right\rangle_{r}
$$

where the angular brackets denote the averaging over all the molecular position in space. From Eq. 5 and Eq. 6, the short time evolution of the ACF will take the form:

$$
g_{0}=\lim _{t \rightarrow 0} g(t)=c n(n-1)\left\langle f_{s}^{2}(\mathbf{r})\right\rangle_{r}+\left(b+c n\left\langle f_{s}(\mathbf{r})\right\rangle_{r}\right)^{2}
$$

when the fluorescent molecule initially is in its ground state $(f(\mathbf{r}, 0)=0)$. At infinite lag times, one finds:

$$
g_{\infty}=\lim _{t \rightarrow \infty} g(t)=\left(b+c n\left\langle f_{s}(\mathbf{r})\right\rangle_{r}\right)^{2}
$$

Similarly, at an intermediate time $t_{i}$, when the molecule is in its photophysical equilibrium, while before the molecular diffusion starts to influence the ACF, there is

$$
g_{i}=c n^{2}\left\langle f_{s}^{2}(\mathbf{r})\right\rangle_{r}+\left(b+c n\left\langle f_{s}(\mathbf{r})\right\rangle_{r}\right)^{2}
$$

Therefore, measuring those three values from a full timescale ACF, the number of fluorescent emitters per molecule can be calculated by using the formula:

$$
\frac{g_{0}-g_{\infty}}{g_{i}-g_{\infty}}=1-\frac{1}{n}
$$

Antibunching measurements is then performed in a way to characterize the dip amplitude (shown in Figure 5) that is antiproportional to the number of independent emitters. Upon the traditional CW excitation, photon emissions from excited molecules occur randomly. It leads to the loss of photons that fall within the detector dead time window and provides insufficient statistics. A more effective measurement of photon pairs can be realized by using pulsed excitation. With a typical $\sim 100$ ps pulse width, a single emitter is excited only once within one pulse. It allows to excite molecules at defined time points and the arrival time of photon detection events can be expected with respect to the pulse 'start' time. In principle, no photon pairs are missing during the dead time of the detector when a sufficiently low laser repetition rate is applied ${ }^{47}$.

When applying pulsed excitation, the photon emission is temporally modulated and the relaxation of $f\left(\mathbf{r}, t_{0}\right)$ should be taken into account. Given the laser pulse width is negligible, 


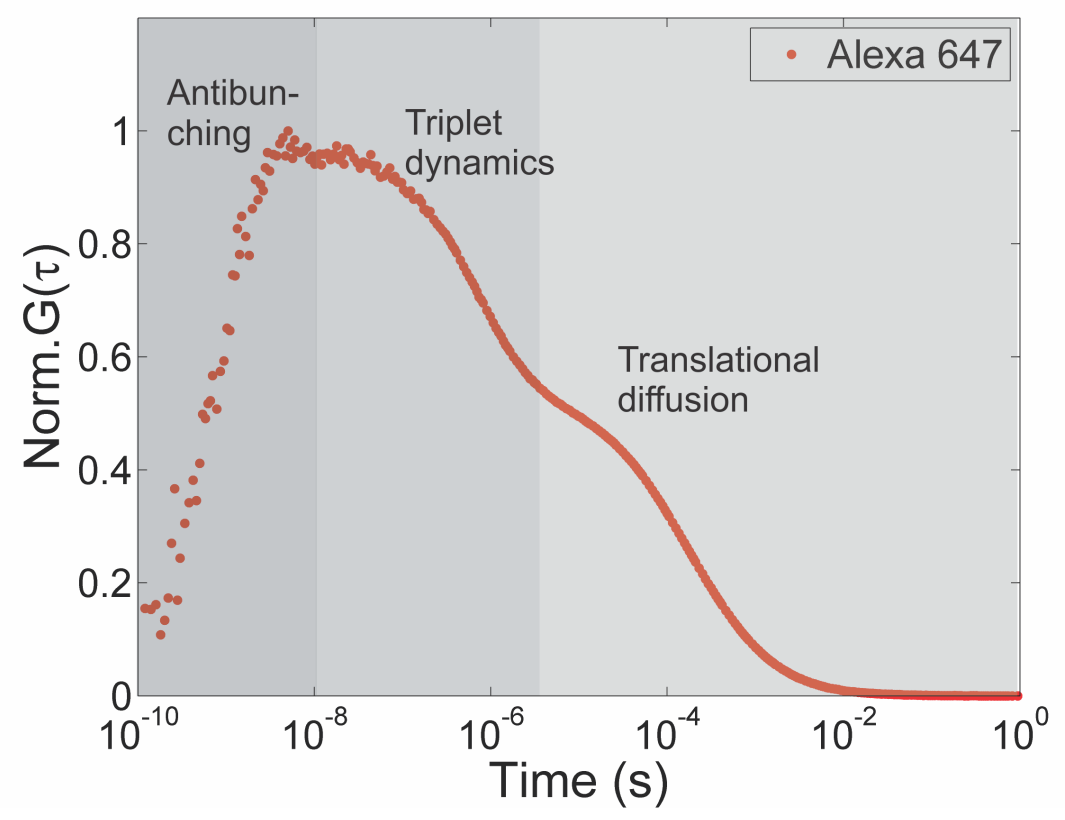

Figure 5: Correlation curve for Alexa 647 dissolved in water, with lag times ranging from nanoseconds to seconds. Different processes contribute to the distinguishable shapes of curve at separable time.

it can then be written as:

$$
f\left(\mathbf{r}, t_{0}\right)=\frac{\kappa_{0}(\mathbf{r})}{\tau}\left(\mathbf{e}^{-\frac{\bmod \left(t_{0}, T_{r e p}\right)}{\tau}}-\mathbf{e}^{-\frac{t_{0}}{\tau}}\right)
$$

where $\tau$ is the fluorescence lifetime and $T_{\text {rep }}$ is the repetition period of laser pulses. Generally, $\tau$ is much smaller than $T_{\text {rep }} . \kappa_{0}(\mathbf{r})$ is a complex position-dependent function that describes the photon detection probability. It takes into account of the excitation intensity distribution, molecular photophysics and system detection efficiency etc. On the long timescale, $f\left(\mathbf{r}, t_{0}\right)$ relaxes toward its stationary function and reads:

$$
f_{s}\left(\mathbf{r}, t_{0}\right)=\frac{\kappa(\mathbf{r})}{\tau} \cdot \mathbf{e}^{-\frac{\bmod \left(t_{0}, T_{r e p}\right)}{\tau}}
$$

Notably, here $\kappa(\mathbf{r})$ is a similar factor as $\kappa_{0}(\mathbf{r})$ while has to take into account the occupation of non-fluorescing states after photophysical relaxation on this timescale.

The ACF is then modulated by the laser pulses and shown as equally separated photon peaks according to the corresponding laser repetitions. From Eq. 11, Eq. 12 and Eq. 6, one can obtain the forms of $g_{0}$ and $g_{\infty}$ through carrying out the averaging and gives:

$$
g_{\infty}=b^{2}+2 b c n\langle\kappa(\mathbf{r})\rangle_{r}+\frac{c^{2} n^{2}\left\langle\kappa^{2}(\mathbf{r})\right\rangle_{r}}{2 \tau} \sum_{j=0}^{\infty} \mathbf{e}^{-\frac{\left|t_{0}-j T_{r e p}\right|}{\tau}}
$$


and

$$
g_{0}=\frac{c}{2 \tau}\left[\left\langle n(n-1) \kappa^{2}(\mathbf{r})+n \kappa_{0}(\mathbf{r}) \kappa(\mathbf{r})\right\rangle_{r} \sum_{j=0}^{\infty} \mathbf{e}^{-\frac{\left|t_{0}-j T_{r e p}\right|}{\tau}}-n\left\langle\kappa_{0}(\mathbf{r}) \kappa(\mathbf{r})\right\rangle_{r} \mathbf{e}^{-\frac{t_{0}}{\tau}}\right]+g_{\infty}
$$

To simplify the expression, antibunching data analysis is performed by fitting the intensity correlation functions simultaneously with models:

$$
A_{\infty}+B_{\infty} \sum_{j=0}^{\infty} \mathbf{e}^{-\frac{\left|t_{0}-j T_{r e p}\right|}{\tau}}
$$

and

$$
A_{0}+B_{0} \sum_{j=0}^{\infty} \mathbf{e}^{-\frac{\left|t_{0}-j T_{r e p}\right|}{\tau}}-C_{0} \mathbf{e}^{-\frac{t_{0}}{\tau}}
$$

Here, those equations include fit parameters $A_{\infty}, B_{\infty}, A_{0}, B_{0}, C_{0}$ and fluorescence lifetime $\tau$. Based on Eq. 13 and Eq. 14, there is the relation:

$$
\frac{B_{0}-B_{\infty}}{C_{0}}=\frac{\left\langle n(n-1) \kappa^{2}(\mathbf{r})+n \kappa_{0}(\mathbf{r}) \kappa(\mathbf{r})\right\rangle_{r}}{n\left\langle\kappa_{0}(\mathbf{r}) \kappa(\mathbf{r})\right\rangle_{r}}
$$

In the case that no significant photophysical process is present, $\kappa_{0}(\mathbf{r})$ and $\kappa(\mathbf{r})$ share the identical physical meaning and have equal values. Thus, it is easy to calculate the number of fluorescent emitters per molecular complex and gives:

$$
n=\left(B_{0}-B_{\infty}\right) / C_{0}
$$

In a more complicated situation, where the fluorescent molecules exhibit additional nonfluorescing states due to triplet state or light-induced cis-trans isomerization, $\kappa_{0}(\mathbf{r})$ and $\kappa(\mathbf{r})$ are not identical. To calculate the number of emitters from Eq. 17 , the ratio between $\left\langle\kappa_{0}(\mathbf{r}) \kappa(\mathbf{r})\right\rangle$ and $\left\langle\kappa^{2}(\mathbf{r})\right\rangle$ need to be determined. As they are describing photon detection probabilities, this ratio can be estimated from the relative correlation amplitudes. The estimation is performed on the timescale where these photophysical processes are involved. Typically, the photophysical kinetics is much faster than the molecular diffusion, and those dynamics are separable in time, allowing to write the ACF for $t \geq 100 \mathrm{~ns}$ as:

$$
g(t)=\alpha \cdot g_{d}(t) \cdot g_{p}(t)+\beta
$$

where $\alpha$ and $\beta$ are fit parameters containing information of sample concentration and background signal. $g_{d}(t)$ is the model function describing the molecular diffusion. $g_{p}(t)$ is the 
photophysical kinetics related part and can be described by exponential decays:

$$
g_{p}(t)=\gamma_{0}+\sum_{i=1}^{N} \gamma_{i} e^{-\frac{t}{t_{i}}}
$$

Here $N$ is the number of components that required to fit the correlation curve, which is essentially associated to the underlying photophysics. Typically, $N=1$ or 2 for organic dyes or fluorescent proteins $\left[\frac{109}{} . \gamma_{i}\right.$ is the amplitude of decay component $i$. One can estimate the ratio between $\left\langle\kappa_{0}(\mathbf{r}) \kappa(\mathbf{r})\right\rangle$ and $\left\langle\kappa^{2}(\mathbf{r})\right\rangle$ as

$$
\frac{\left\langle\kappa_{0}(\mathbf{r}) \kappa(\mathbf{r})\right\rangle}{\left\langle\kappa^{2}(\mathbf{r})\right\rangle}=\frac{\sum_{i=0}^{N} \gamma_{i}}{\gamma_{0}}
$$

Therefore, from Eq. 17 and Eq. 21, the number of emitters $n$ can be calculated from the modified formula of Eq. 18, which is given as:

$$
(n-1) \frac{\gamma_{0}}{\sum_{i=0}^{N} \gamma_{i}}+1=\left(B_{0}-B_{\infty}\right) / C_{0}
$$

\subsection{Experimental: probing the stoichiometry of single-molecule and determining molecular labeling efficiency}

Photon antibunching measurement is experimentally realized in a Hanbury Brown-Twiss (HBT) setup 11, where the photon flux is equally split and detected by two detectors. Here, successive photons hitting on those two detectors are recorded through a Time-correlated single photon counting (TCSPC) electronics with picoseconds temporal resolution. The HBT-type measurement with two detectors has benefits of avoiding the influence from the dead time in single detector case and also enabling a high detection count rate. Photon counting events are stored as a series of arrival times and can further be cross-correlated in the picosecond to nanosecond regime for statistical analysis.

Figure 6 shows the schematic of HBT-type confocal-based fluorescence microscopy system used for photon antibunching measurements. Briefly, the $40 \mathrm{MHz}$ circular polarized pulsed ( 50 ps FWHM) laser of $640 \mathrm{~nm}$ (LDH-D-C-640, PicoQuant) is guided by a polarizationmaintaining fiber before collimation. The beam diameter is adjusted to $8 \mathrm{~mm}$ by a ringactuated iris diaphragm and then reflected by a dichroic mirror (FITC/TRITC, Chroma Technology) towards the microscope's objective (UPLSAPO $60 \times \mathrm{W}, 1.2$ N.A., Olympus). Fluorescence is collected by the same objective, passed through the dichroic mirror, and 
focused onto a single circular aperture (diameter $150 \mu \mathrm{m}$ ). After the pinhole, the emission light is split by a 50/50 beamsplitter, and filtered by band-pass filters (BrightLine HC 692/40, Semrock) before refocused onto two single-photon avalanche diodes ( $\tau$-SPAD, PicoQuant) respectively. TCSPC electronics (HydraHarp 400, PicoQuant) records detected photons from both detectors with an absolute temporal resolution of 2 ps.

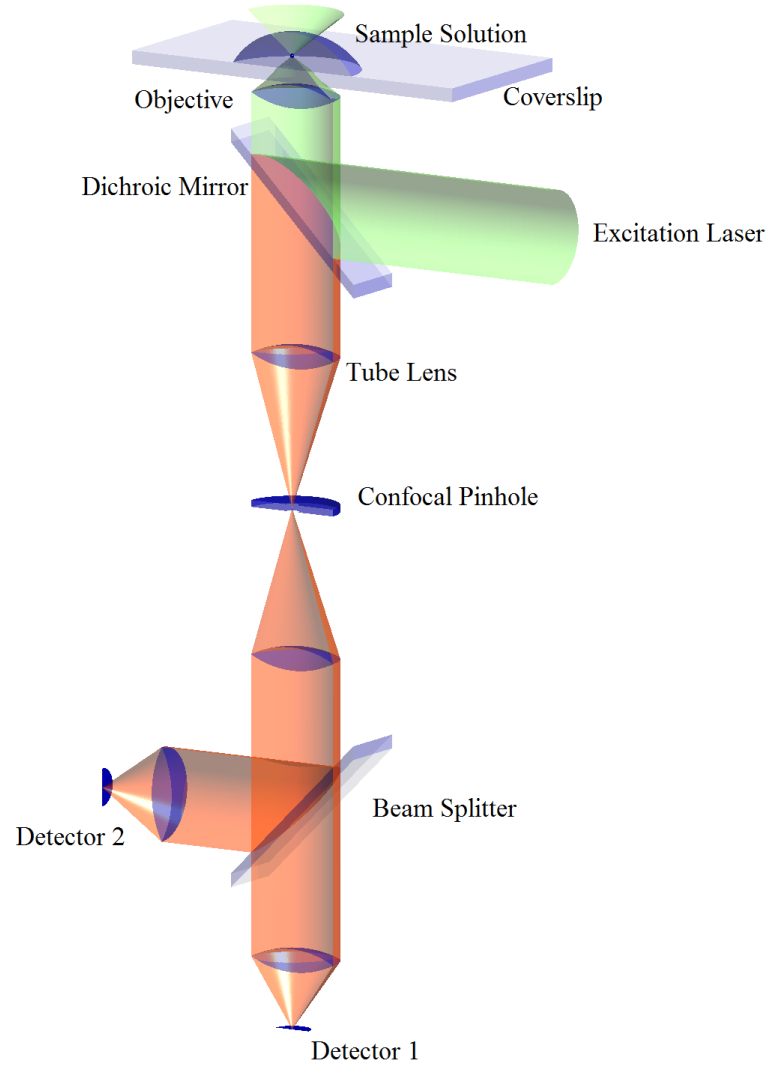

Figure 6: Schematic of confocal microscopy for photon antibunching measurements

To mimic the protein oligomerization process, double-stranded Deoxyribonucleic acid (dsDNA) fragments are chemically modified to contain different numbers of covalent binding sites. These modified sites were labeled using the fluorescent dye Atto 647N. The samples are purified to get rid of free Atto $647 \mathrm{~N}$ in buffer solution. An 8-well tissue culture chamber on a $170 \mu \mathrm{m}$ coverslip (Sarstedt) is used for all the measurements. To avoid adsorption of the dsDNA on surface, the chamber is cultured with $0.3 \mathrm{mg} / \mathrm{ml}$ BSA for 20 minutes before 
measurements. Afterwards, the passivated chamber is filled with $200 \mu \mathrm{L}$ solution of dsDNA sample for antibunching measurement. In all measurements, the excitation light is focused in the solution $50 \mu \mathrm{m}$ on top of the coverslip. The excitation power is $\sim 20 \mu \mathrm{W}$ before the objective. The sample concentration is adjusted to have on average 2 to 8 molecules in the confocal detection volume. This gives a good SNR and ensures the linear working performance of both detectors. Each sample is measured for 20 minutes at a constant temperature of $22{ }^{\circ} \mathrm{C}$. The photon detection events are stored and then processed with a custom Matlab routine.
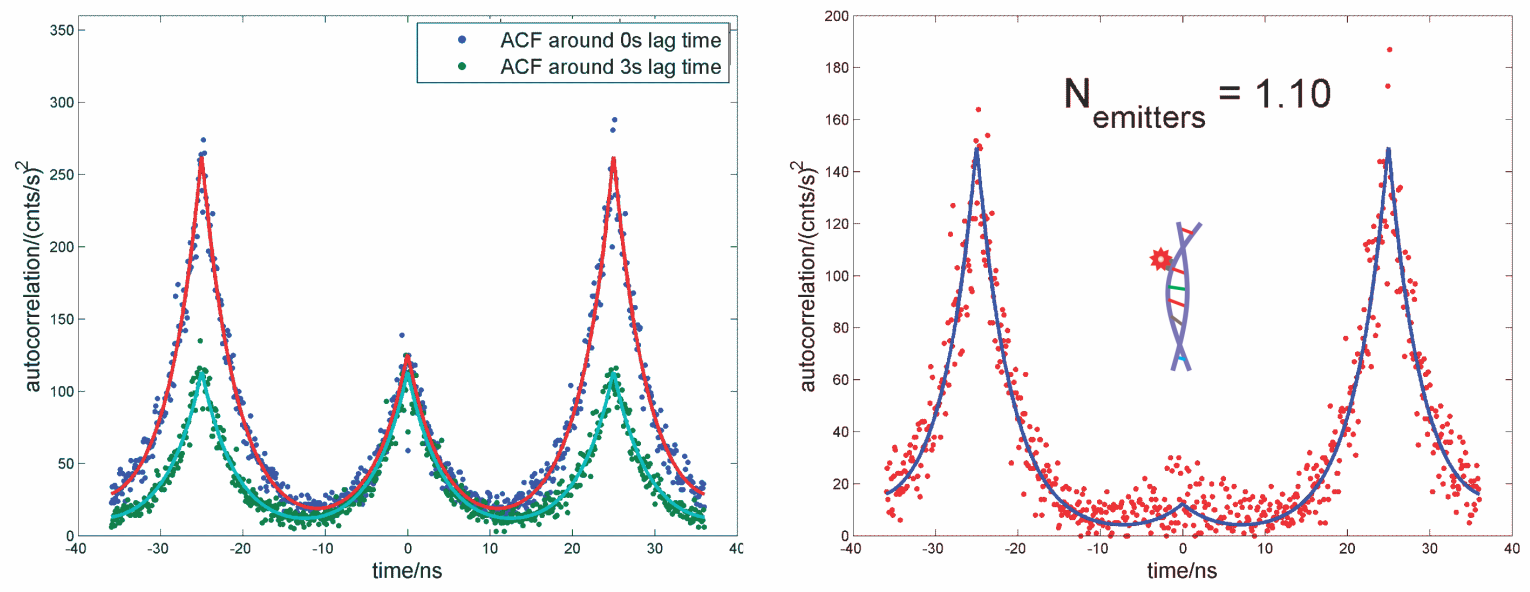

Figure 7: Correlation functions (dots) and the curve fit (solid lines) from the measurement of single Atto $647 \mathrm{~N}$ labeled dsDNA. The figure on the left compares the ACFs at lag times around 0 $\mathrm{s}$ and $3 \mathrm{~s}$, on the right side is the result obtained from the subtraction of two ACFs.

The cross-correlation functions between two channels are calculated around two different lag times, corresponding to ACFs of $g_{0}$ and $g_{\infty}$. Figure 7 shows the photon correlation results with a 200 ps time bin for single Atto 647N labeled dsDNA, both around $0 \mathrm{~s}$ lag time (blue dots) and $3 \mathrm{~s}$ lag time (green dots). The correlation function around $3 \mathrm{~s}$ lag time represents the same meaning as $g_{\infty}$ since the single-molecule correlation $g_{1}$ has completely decayed to zero on this timescale. In all the correlation figures, the data is shown on a symmetric positive and negative lag time range, corresponding to the photon pair correlation from the first detector against the second detector and from the second detector against the first detector, respectively. It is noticeable that even though the pure single labeled sample is 
measured, a non-zero value is obtained at zero lag time. It is due to the scattering and uncorrelated detector background. Since these also equally contribute to the ACF at lag times around $3 \mathrm{~s}$, it can be corrected. The right panel of Figure 7 shows the correlation function after the subtraction, which represents the contribution only from the molecules that passed through the detection volume. Models from Eq. 16 and Eq. 15 are then applied to fit the two correlation functions simultaneously with fit parameters $A_{\infty}, B_{\infty}, A_{0}, B_{0}, C_{0}$ and fluorescence lifetime $\tau$.

After the correction, it is noted that the correlation peak at lag time zero has diminished to the background level and a calculated emitter numbers of 1.1 based on Eq. 22 is achieved. It is nicely in agreement with the value one as expected.
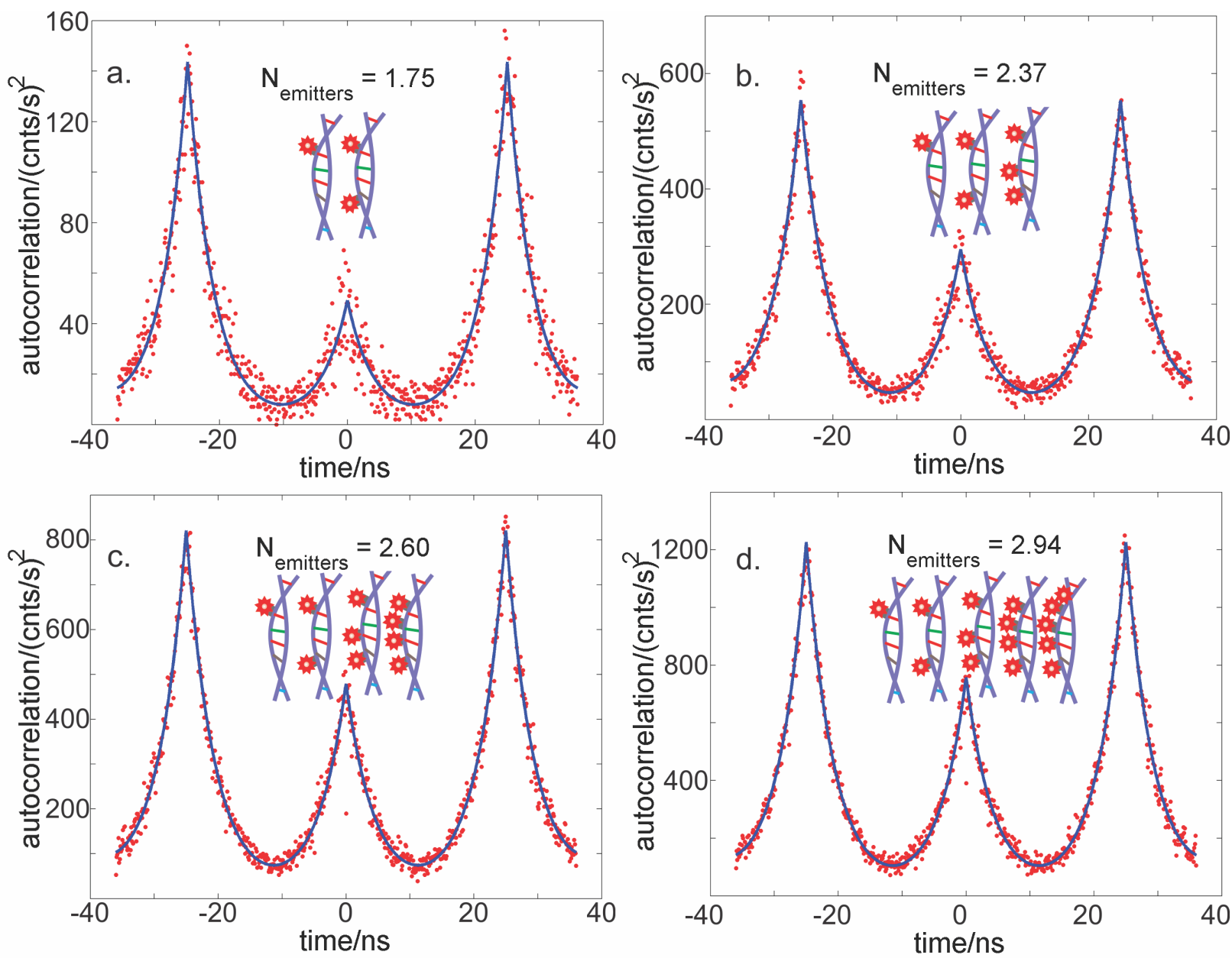

Figure 8: Correlation functions (red dots) and the fits with model. The average emitter numbers is then calculated for the case of (a) mixture of single and double Atto 647N labeled dsDNA and the maximum (b) three, (c) four, (d) five Atto 647N labeled dsDNA. 
On the other hand, from the same photon counting data, the ACF at lag times in the range from $100 \mathrm{~ns}$ to $1 \mathrm{~s}$ is also calculated. Here, the fluorophore photophysical relaxation is demonstrated. Fitting the curve based on Eq. 20, one obtains the correction factor $\frac{\sum_{i=0}^{N} \gamma_{i}}{\gamma_{0}}$ for calculating the number of emitters. Typically, the total photon counting records are divided into several bunches (e.g. 10,000,000 photon counts each) and a bootstrapping algorithm is then used. It repeatedly resamples those bunches to produce a new averaged FCS curve and thus be fitted. Multiple fitting results are achieved that allows an estimation of the fitting error.

To check if this method is capable of determining the number of emitters, dsDNA samples with two, three, four and five binding sites are measured. In all those cases, each sample is composed of different labeled species depending on the labeling efficiency. For instance, the sample of three binding sites contains the fluorescent species of one, two and three dyes labeled dsDNA, as illustrated in Figure 8(b). In Figure 8, the increasing peak amplitudes at zero lag time going from panel (a) to panel (d) matches well with the sample compositions and the average numbers of emitters per complex.

Furthermore, from this average number, statistical analysis can unveil more information about the molecular complex. An example is the labeling efficiency, which refers to the probability of a binding site being labeled. Given that a molecular complex contains the maximum $N$ emitters (i.e. $N$ binding sites), the probability for a molecule to contain $k$ emitters is represented as $P(k)$. Thus, from Eq. 17, the observed average number of emitters has the form of

$$
n=\frac{\sum_{k=0}^{N} P(k) \cdot k^{2}}{\sum_{k=0}^{N} P(k) \cdot k}
$$

For a constant labeling efficiency of $\varepsilon$, the $p(k)$ equals the binomial distribution $B(N, k)$. The mean and variance of this distribution are:

$$
\mathrm{E}(k)=\sum_{k=0}^{N} P(k)=N \cdot \varepsilon
$$

and

$$
\operatorname{Var}(k)=\sum_{k=0}^{N} P(k)(k-E(k))^{2}=N \cdot \varepsilon(1-\varepsilon)
$$

Thus Eq. 23 can be simplified as:

$$
n=1+(N-1) \cdot \varepsilon
$$


which gives a direct way to calculate the labeling efficiency from the observed average number of emitters. From the previous results, it gives

\begin{tabular}{ccccc}
\hline Binding sites $(\mathrm{N})$ & 2 & 3 & 4 & 5 \\
\hline Emitter numbers $(\mathrm{n})$ & 1.75 & 2.34 & 2.72 & 2.90 \\
\hline Labeling efficiency $(\varepsilon)$ & 0.75 & 0.68 & 0.57 & 0.48 \\
\hline
\end{tabular}

where the more binding sites present the smaller is the achieved labeling efficiency.

\subsection{Discussion}

The presented results show that the exploration of photon antibunching is not limited to a proof of single emitter, but can be a valuable tool for quantitative spectroscopy of biomolecules. By applying pulsed excitation and state-of-the-art TCSPC electronics, rich information of photon statistics is recorded that can simultaneously monitor important fluorescence properties such as intensity and fluorescence lifetime.

In comparison to alternative methods (e.g. $\mathrm{PCH}$, FIDA) that distinguish molecular species based on the fluorescent intensity fluctuations, photon antibunching analysis takes into account of the temporal behavior of photon counting and offers a reference-free investigation. For example, Ly and coworkers have compared the photon antibunching and $\mathrm{PCH}$ in the study of determining the number of apolipoprotein A-I molecules bound to high-density lipoprotein and obtained the same stoichiometry of two ${ }^{[78}$. However, the $\overline{\mathrm{PCH}}$ method requires additional calibration using a single labeled control sample to work. Moreover, the PCH data analysis involves a careful determination of detection volume, which is sensitive to many experimental conditions, e.g. coverslip thickness, refractive index of the solution, or saturation ${ }^{36}$. In contrast, the photon antibunching approach is simpler and is immune to changes of the detection volume. The data analysis only includes parameters of intrinsic fluorescent properties of the molecule. It has also shown that the photophysical behavior of different dyes can be well-determined and the remaining uncertainties do not noticably influence the determined stoichiometry.

Nevertheless, from Eq. 10, the theoretical signature of $n$ emitters in a correlation analysis (i.e. the difference in correlation amplitudes) is proportional to $1-1 / n \underline{47}$. In practice, the feature becomes already very difficult to be discerned where more than four emitters 
present in a complex. It means that the characterization ability is degrading when going to higher labeling stoichiometry. The molecular brightness-based method would possibly have better performance in this case ${ }^{78}$. However, a potential solution is to investigate higher orders of correlation, e.g. third-order correlations and fourth-order correlations. The physical understanding behind is to investigate the probability of detecting 3 or 4 photons from one molecule after single pulse excitation. Therefore, more detection channels are required for counting those coincident photons. The similar concept has been applied to count the label number of immobilized DNA molecules and realized up to the stoichiometric number of $\sim 20[110[111$. But an analytical method for in solution studies has never been proposed or experimentally realized till now.

It is also noticeable that this photon statistics will fail to estimate the molecular stoichiometry if the labels are in close proximity and energy transfer between the emitters is pronounced 1095 [55. Another issue is that the stoichiometry is achieved by accumulating the photon statistics from many molecules. Therefore, the method only gives an average number of emitters per molecule, which limits the application to samples of rather homogeneous labeling stoichiometry ${ }^{109}$. In order to disentangle the heterogeneously labeled molecular complexes and to measure the full distribution, an isolated investigation of each molecule is required and more photon statistics is needed. For this purpose, another method of imagingbased photon counting histogram assisted by a nanofluidic device is developed and will be discussed in details from the next chapter. 


\section{Single-molecule detection and manipulation in nanoflu- idic channels}

\subsection{Nanoconfinements in single-molecule detection}

Single-molecule fluorescence microscopy and spectroscopy enable deep insights into biological processes. Due to the development of bright and stable fluorescent probes that can be used to label target biomolecules, fluorescence microscopy is highly selective. The photophysical properties of dyes, such as brightness, excited state lifetime, spectra, etc., are highly sensitive to their local environments, which makes them excellent molecular nanoprobes.

The fluctuations in the local environment can be monitored by observing the variations in the photophysical properties of each single emitter. This requires an optical technique that can illuminate single molecules selectively for a long duration. Conventional widefield microscopy delivers no optical sectioning, and the fluorescence from a broad field of view is observed simultaneously, which reduces the SNR, Techniques such as Light sheet fluorescence microscopy (LSFM) [63] [108] and TIRF microscopy [4] provide the required optical sectioning, but cannot be used to study single-molecule spectroscopy due to their slow camera frame acquision rates and fast diffusion of the molecules. To date, confocal microscopy has held a great promise in such single-molecule experiments due to its focused excitation spot which is diffraction limited (on the order of half a wavelength), single photon detectors that have high timing accuracy and high detection efficiency, and the ability to block background and out-of-focus fluorescence (optical sectioning). A variety of tools such as FCS, Fluorescence Lifetime Correlation Spectroscopy (FLCS) 61$]$ exist that use confocal excitation and detection to study protein conformational dyanimcs, binding/unbining dynamics and aggregation at single-molecule level. However, one of the major disadvantages is the residence time of the molecule in the focus, which is on the order of a milisecond. Only the average properties of the molecules measured through the focus of excitation are measured.

Recently, several approaches to confine the diffusion space of the molecules have been developed, in order to increase their observation times, such as nanocontainers, convex lensinduced confinement, Nanochannels, etc. which will be discussed briefly below. The core idea is to increase the single-molecule observation time, without having the need to immobi- 
lize them on the surface or tether them, which might interfere with the molecular dynamics. The concept of nanoconfinement is a powerful tool for single molecule spectroscopy and has been realized in various ways $[$.

\subsubsection{Nanocontainers}

Methodological developments of nanoconfinement have been continued for last decades alongside research of single-molecule techniques. One approach uses the idea of so-called nanocontainers, which are taking advantage of the formation of nano-sized lipid vesicles [87] [10] or droplets 44 to encapsulate the individual molecules in a confined space. The surrounding solvent condition of encapsulated molecules remains similar to the bulk sample. In this scenario, movement of the small molecule is coupled with the slow motion of its encapsulator.
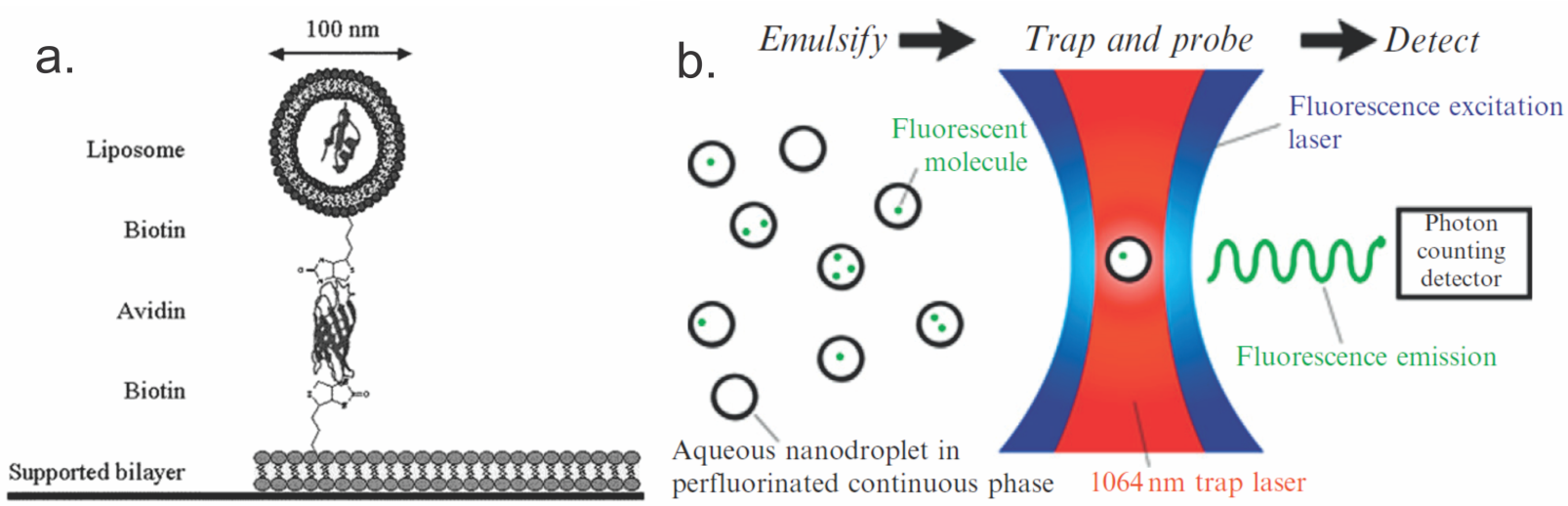

Figure 9: Two types of nanocontainers: a. To extend the measurement time of individual molecules by phospholipid vesicles encapsulation. The streptavidin-biotin chemistry is used here to tether the vesicle to the surface ${ }^{[10]} ; b$. Nanodroplets generated from emulsification can be trapped using an optical tweezer, and the single-molecule in the nanodroplet can then be observed for long

$$
\text { time } 44 \text {. }
$$

Since the molecule of interest is incomparable with the nanocontainer in size, its rotational degree of freedom is only minimally influenced. The diameter of a typical nanocontainer is around 2 to 3 orders of magnitude larger than that of studied single molecules ${ }^{[69}$, so the observation time of these molecules can then be extended by 100 to 1000 times. Further methods such as surface tethering or optical trapping that are not compliant with small 
molecules can now be applied to slow down or even fix the motion of nanocontainers, as shown in Figure 9. This can further extend the observation time of single molecules. However, though this method has shown its applicability in studying soluble proteins $\underline{87}$, the non-trivial sample preparation for desirable yield and the difficulty in providing buffer exchanges make it impractical for broad biological applications.

\subsubsection{Micro/nanofabricated platforms}

The technique of micro/nanofabrication also offers opportunity for long-term observation of single molecules in solution. As the devices are typically made of optically transparent materials, e.g. silicon dioxide, Polydimethylsiloxane (PDMS) and Poly(methyl methacrylate) (PMMA), they are ready to combined with the optical detection. When the dimension of these devices is scaled down to the range of hundreds of nanometers, along the direction of optical axis for a high NA objective, molecules moving inside are always seen in focus.

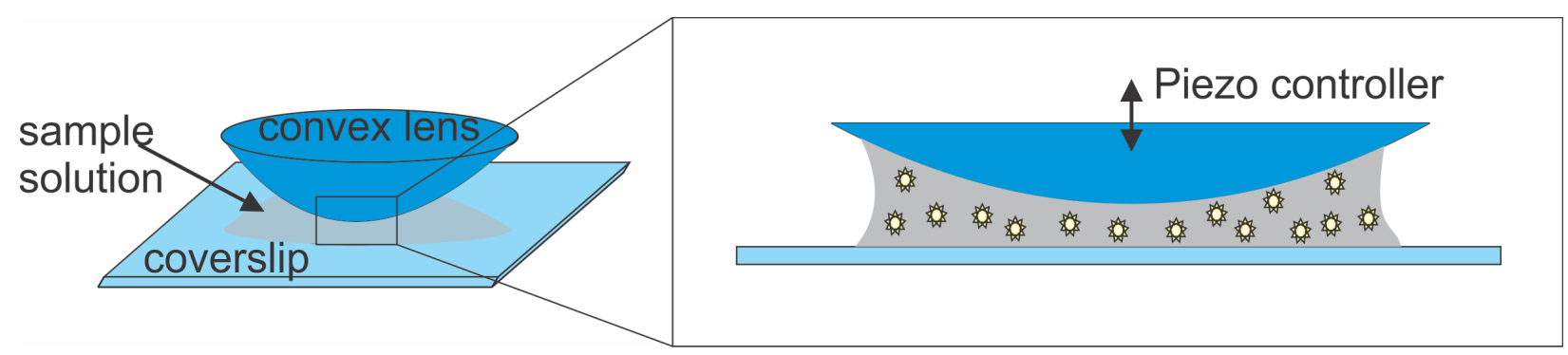

Figure 10: Illustration of CLIC. Molecules in solution are confined in a thin layer between a plano-convex lens and coverslip surface (left). The confinement is regulated by a Piezo controller that can adjust the thickness of this layer (right).

A simple method known as convex lens-induced confinement (CLIC) has been developed to achieve this goal $\underline{69}$. As illustrated in Figure 10, A. Cohen and coworkers take use of the curved surface of a plano-convex lens that is in close vicinity to the top surface of a microscope coverslip. It can squeeze molecules in the gap of two sheets down to a dimension of several nanometers ${ }^{69}$. Molecules from the bulk solution can enter into this gap through diffusion and they are kept in the focal plane till diffusing out. 


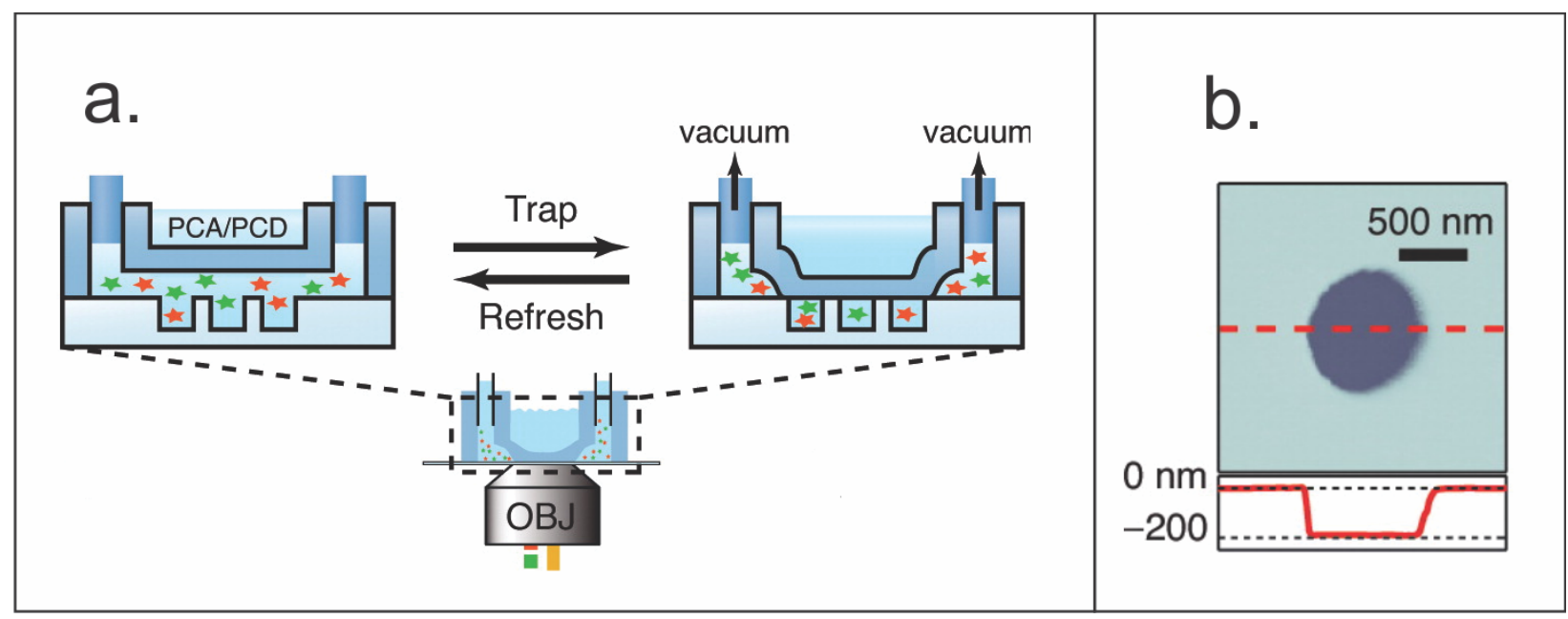

Figure 11: Molecules enter into the nano-dimples array are trapped. (a) A polymer lid is used to seal the dimples reversibly. (b) Atomic force microscope image shows the size of a dimple. It has the radius of ca. $450 \mathrm{~nm}$ and height ca. $200 \mathrm{~nm}$. Adapted from ref. [105].

In CLIC, the design can be conveniently adapted to a conventional inverted fluorescence microscopy and is suitable for camera-based imaging. In their study, diffusing lipid vesicles have been recorded for a time that is 10,000-fold longer than a confocal-based setup 69 . For small molecules such as short DNA strands, another method is also developed by the same group. There, nanofabricated holes or 'dimple' are applied to trap the individual molecules locally 105 . This idea of confinement is similar to the concept of aforementioned vesicle or droplet nanocontainers, expect that the 'dimples' can undergo reversible sealing with the aid of a pneumatically controlled PDMS lid, as shown in Figure 11. With several hundred nanometers in diameter and $200 \mathrm{~nm}$ in depth, the 'dimples' allow fluorescent molecules to be trapped and probed similar to immobilized molecules.

Though CLIC is a cheap and well-established design, it is not as desired to actively transport molecules. The way of molecules stochastically diffusing in and out of the confined region limits the throughput of this system ${ }^{5}$. Nevertheless, CLIC and 'dimples' ideas lead the way to perform on-chip single-molecule studies. Nanoconfinements can also be realized on an micro/nanofluidic system, which can be finely tailored for precise single-molecule manipulation and rapid detection 127 . This vivid research area is motivated by integrating chip-like devices in a manner analogous to developed microelectronics. Therefore, the analysis of bioanalytes can be accomplished with high throughput. It holds a great potential for massively 
parallel diagnostics ${ }^{30}$. The state-of-the-art micro/nanofabrication techniques enable the format of sophisticated nanoconfinement on an optically transparent material such as glass or PDMS. Depending on the requirements, various fabrication strategies can be designed for the fluorescence-based single-molecule studies [12] 82 [1] 32 .

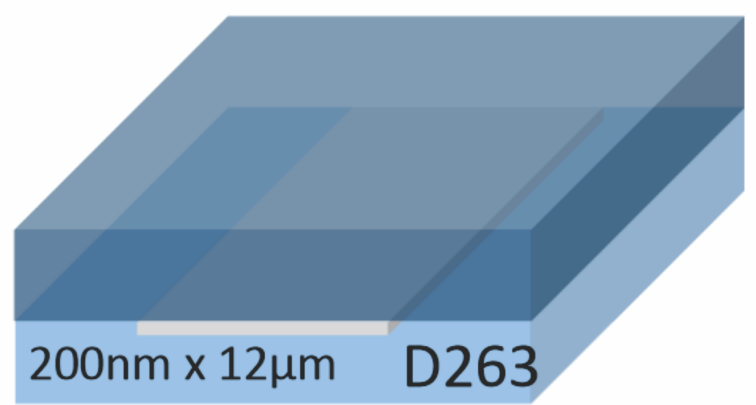
2D nanochannel

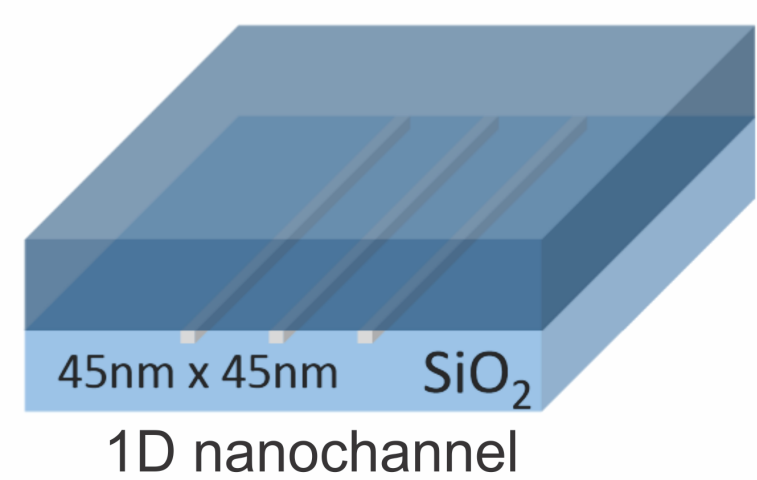

1D nanochannel

Figure 12: Two types of Nanochannels that are applied for single-molecule detection. Both can confine the molecular motion in a plane with height smaller than $200 \mathrm{~nm}$.

As shown in Figure 12, the sheet-like (2D Nanochannels) or line-like (1D Nanochannels) nanoconfinement can be applied to guide the transport of molecules. 2D Nanochannels devices are routinely fabricated through conventional optical lithography in combination with finely controlled wet etching. A patterned chip with inlet/outlet is afterwards bond to a microscope coverslip for further applications. In this case, molecules in the channel imaged by microscope objective are seen as moving in a $2 \mathrm{D}$ plane. Concerning the more confined case of 1D Nanochannels, patterns width has a dimension smaller than the half wavelength of visible light. Therefore, optical lithography can not work on this precision and electronbeam lithography is commonly applied, which will dramatically increase the fabrication cost. Many emerging techniques can facilitate the fabrication without costly lithography process, such as using nanofluidic optical fiber ${ }^{39}$ or PDMS patterning and adhesive bonding [121 [112]. As demonstrated in Figure 13, a procedure of making the low price micro/nanofluidic chip with minimized requirement of cleanroom fabrication is proposed based on my primary tests. PDMS is used as both the building materials and the sealing agent, and is stamped 
on a microscope coverslip to produce channel patterns. No etching process is required for chip fabrication. Further improvement in line of this study can facilitate the integration of nanofluidic devices into microfluidic systems 112 .

\section{Clean coverslip}

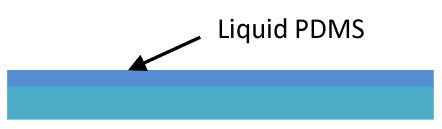

2. Spin coat a thin layer of liquid PDMS (diluted in Hexane)

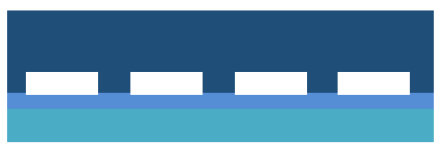

3. Place a channel-patterned PDMS onto the coated coverslip

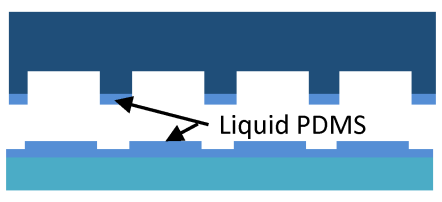

4. Peel off the channel-patterned PDMS and place it onto another clean coverslip

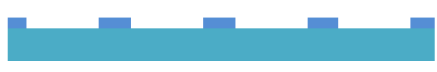

5. Peel off the channel-patterned PDMS and form a liquid PDMS pattern

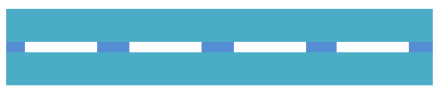

6. Plasma bond with another clean coverslip to form a nanofluidics chip.

Figure 13: A proposed method for fabricating low price chip by applying PDMS patterning and adhesive bonding.

Benefiting from the precise feature of nanofluidic devices and underlying nanoscale phenomena such as electrodynamics, sterics, and adsorption dynamics ${ }^{[86}$, versatile single-molecule applications can be realized. In all these designs, at least one dimension of molecular motion is physically confined, and different single-molecule detection strategies can be utilized. 


\subsection{Single-molecule detection in 1D/2D Nanochannels}

\subsubsection{Confocal-based detection}

H. G. Craighead and co-workers have pioneered the application of submicrometer-sized fluidic channels for single-molecule based epigenetic analysis 11 18. At the nanoscale, electric fields are widely applied to drive flow and affect the transport or separation of biological mixtures, which can be developed into novel detection or manipulation methods $\frac{86}{}$. In particular, ABEL trap enables to trap individual fluorescent biomolecules in an aqueous solution. Therefore, immobilization-based single-molecule analysis can be directly implemented to probe long time single-molecule fluorescence signal in solution. It provides new insights of biomolecular dynamics, e.g. protein folding, single-molecule binding, and conformational changes, without the possibly perturbative interaction from the surface 23] 20] 21] [40]. Those works on small fluorescent molecules have been performed by using the confocal-based pointlike detection. There, single-molecule detection events are manifested as individual intensity bursts on a time trace of the fluorescence signal. Using high-speed single photon counting electronics, the confocal-based detection provides advanced spectroscopic modalities for comprehensive single-molecule studies.

As shown in Figure 14, experiment using a confocal microscope to detect single molecules has been performed within 1D Nanochannels (Bionano Genomics). The intensity time trace of molecules inside and outside the Nanochannels can be analyzed by FCS, providing information of molecular mobilities. The models in each case are written as:

$$
\text { Free solution: } g_{d}(t)=\frac{1}{N} \cdot \frac{1}{\sqrt{1+4 D t / w_{x}^{2}}} \cdot \frac{1}{\sqrt{1+4 D t / w_{y}^{2}}} \cdot \frac{1}{\sqrt{1+4 D t / w_{z}^{2}}}
$$

and

$$
\text { 1D-Flow: } g(t)=\frac{1}{N} \cdot \frac{1}{\sqrt{1+4 D t / w_{x}^{2}}} \cdot e^{-\frac{t^{2}}{\left(\frac{w_{x}}{v_{x}}\right)^{2}\left(1+4 D t / w_{x}^{2}\right)}}
$$

where $w_{i}$ is the $e^{-2}$ radius along each axis $i$ of the excitation geometry, $N$ is the average number of molecules in the detection volume, $v_{x}$ is the average flow velocity of the fluorescent sample and $D$ is the diffusion coefficient of molecules. In Figure 14 A, the FCS analysis of single Atto $647 \mathrm{~N}$ labeled dsDNA (22bp) undergoing diffusion in free solution and 1D flow transport in Nanochannel is compared. The correlation results are both well fitted by 
the corresponding models. A flow rate of ca. $1 \mathrm{~mm} / \mathrm{s}$ is calculated for measurement in Nanochannels.
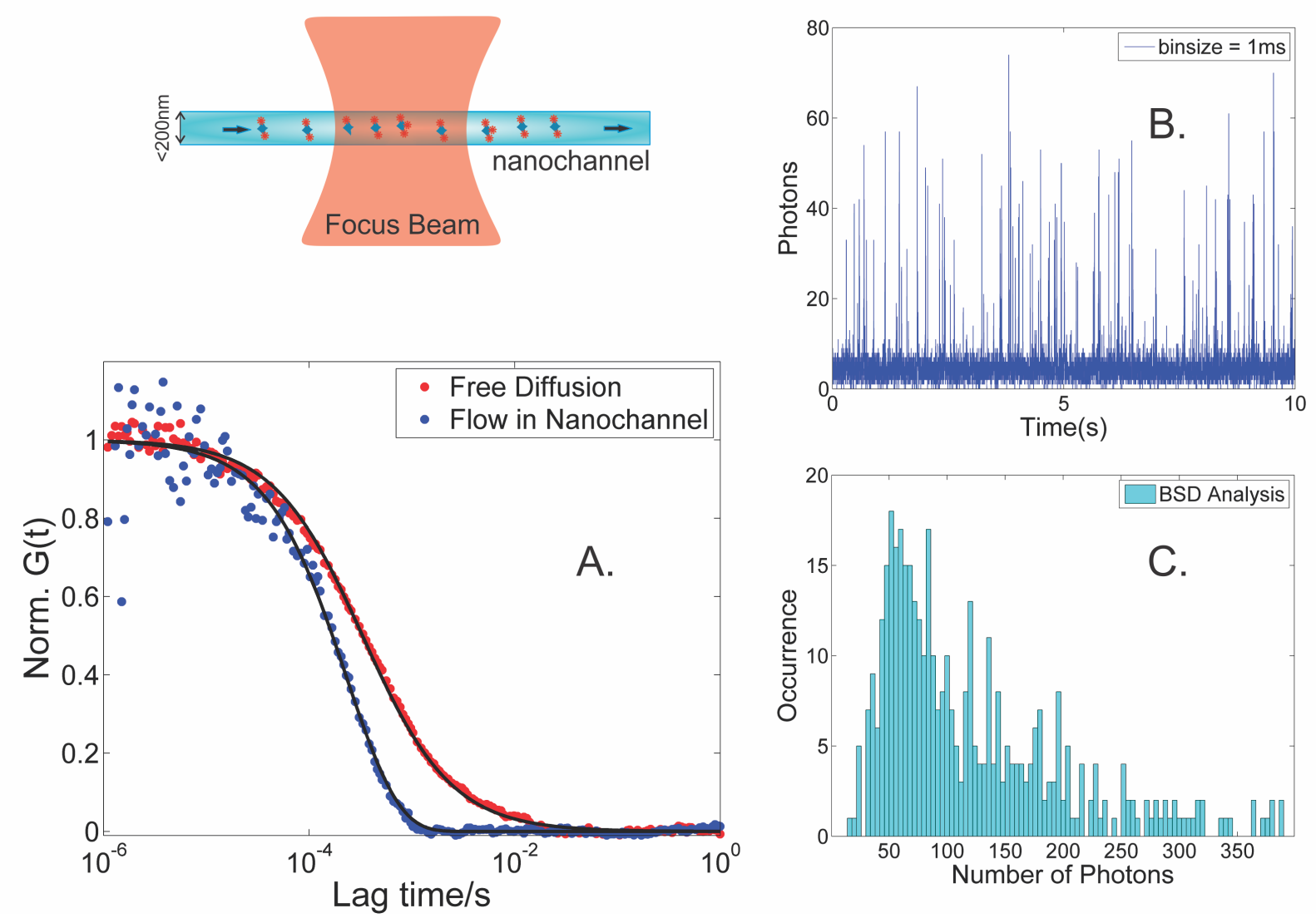

Figure 14: Confocal-based single-molecule detection in 1D Nanochannels. (A) Comparing molecular motion inside and outside of Nanochannels. Sample Atto $647 N$ labeled dsDNA in solution is measured and analyzed by FCS.(B) Typical fluorescent intensity bursts of dsDNA measured in 1D Nanochannel. (C) BSD analysis of intensity bursts from (B).

This experimental configuration is further applied to perform the BSD analysis as shown in Figure 14 B and $\mathrm{C}$, where each intensity burst is regarded as one single-molecule detection event and the photon counts contained in each burst are integrated. The BSD is then calculated from thousands of single-molecule detection events and is exhibited as a photon counting histogram shown in Figure 14 C. A direct interpretation of this histogram would suggest the presence of more than one species of molecules, though the measurement is indeed conducted only on single species. The additional distribution at the region of high photon counts is an effect of the so-called molecular noise that results from multi-molecule 
burst events, which means two or more molecules passing in close succession through the detection volume but yields only one single photon burst 37 38. As single-molecule detection events are deduced indirectly, discerning multi-molecule burst events is very difficult in this experimental configuration. Though sample dilution can alleviate this effect, the analysis throughput would be dramatically reduced. Hence, to facilitate the brightness analysis into a more practical platform, I turned to develop an imaging-based method that can directly observe individual molecules in solution.

Imaging-based methods enable the parallel investigation of multiple molecules at one time, which is in concert with the high-throughput analysis capability of the micro/nanofluidic system. Compared with the confocal-based detection, the imaging-based method would have to sacrifice in time-resolution due to the limit of camera read-out rate. However, the visualization and localization of single molecules from an image frame reveals molecular properties in a more straightforward manner.It holds the potential to develop into an analytical tool that has fewer experimental constraints than confocal-based method. Large micrometer-sized DNA strands have been stretched and successfully imaged in nanofluidic channels before 48 , but the imaging of fast diffusing small molecules has not yet been reached till now.

\subsubsection{Imaging-based detection}

With the advent of sensitive cameras such as EMCCD, it is possible to record the fast moving single-molecule in Nanochannels. The main concern is collecting enough photons (i.e. photon budget) from the individual molecules. Using nanoconfinement, molecules can be kept in focus and the out-of-focus background is tremendously reduced. Photons coming from individual molecules would spread onto the camera pixels as patterns described by a PSF. Each molecule is seen as individual PSF on an recorded image. The real molecule position can be re-mapped from those PSFs, nevertheless, with an uncertainty that is related to the information including collected number of photons for each molecule, background and molecular motion.

For example, in a short detection time window of $1 \mathrm{~ms}$, in practice, ca. 100 photons are 
needed for properly visualizing a single-molecule. Thus, the systematic count rate should be larger than $10^{5}$ count/s. It demands a rather high excitation power density on the objective focus plane. Since a system of high-throughput relies on a large illumination size in order to record more molecules in parallel, the total input power that enters into the objective should be sufficiently high. Particularly, when quantitative microscopy (e.g. molecular brightness analysis, see in Chapter 4) is going to be performed, a homogeneous excitation is required. Under a typically wide-field illumination condition, the input beam is focused at the back focal plane (BFP) of the applied objective. In the vicinity of the first lens surface close to the BFP of objective, power density is very high. Careful consideration and compromise should be taken to avoid the potential damage of optical components. In practice, a power density of ca. $100 \mathrm{~kW} / \mathrm{cm}^{2}$ in a ca. $10 \mu \mathrm{m} \times 10 \mu \mathrm{m}$ illumination area can be securely working on a conventional microscope objective for typical organic dyes (e.g. Cy5, Atto655, Atto647n, etc.). The later applied pulsed excitation in stroboscopic imaging (Section 4.1) can help avoid the potential damage.

In the first generation of the imaging system, I employed a beam shaping design to generate a sheet-like illumination that can selectively excite molecules in the single Nanochannel. The beam profile is coupled to the objective diagram to make the most of input energy. In Figure 15 A, the incident laser is shaped as a rectangular flat-top beam by using a Powell lens (PL) ${ }^{14}$ and beam size is further adjusted by using a different combination of Cylindrical lens (CL) and telescope lens pairs. There, multiple images of fluorescence signal under this illumination condition are averaged to demonstrate the excitation profile in one channel. Figure $15 \mathrm{~B}$ and $\mathrm{C}$ compares the single frame image of $5 \mathrm{nM}$ STAR 635P (Abberior GmbH, Goettingen) labeled dsDNA diffusion outside and inside the Nanochannels under constant experimental conditions. As it demonstrates, the nanoconfinement benefits single-molecule detection by largely suppressing the out-of-focus fluorescence background and isolating molecules spatially. Figure $15 \mathrm{D}$ is the averaged image from multiple frames of single molecules imaging in one Nanochannel, displaying the molecule transits along the channel direction. The system can also work on a traditional Gaussian illumination, where the PL and CL lens need to be replaced by a telescope lens. It allows for investigation of molecules in multi-channels that improves the analysis throughput.

Though the moving molecule can already be evidently observed inside the nanofluidic device, 

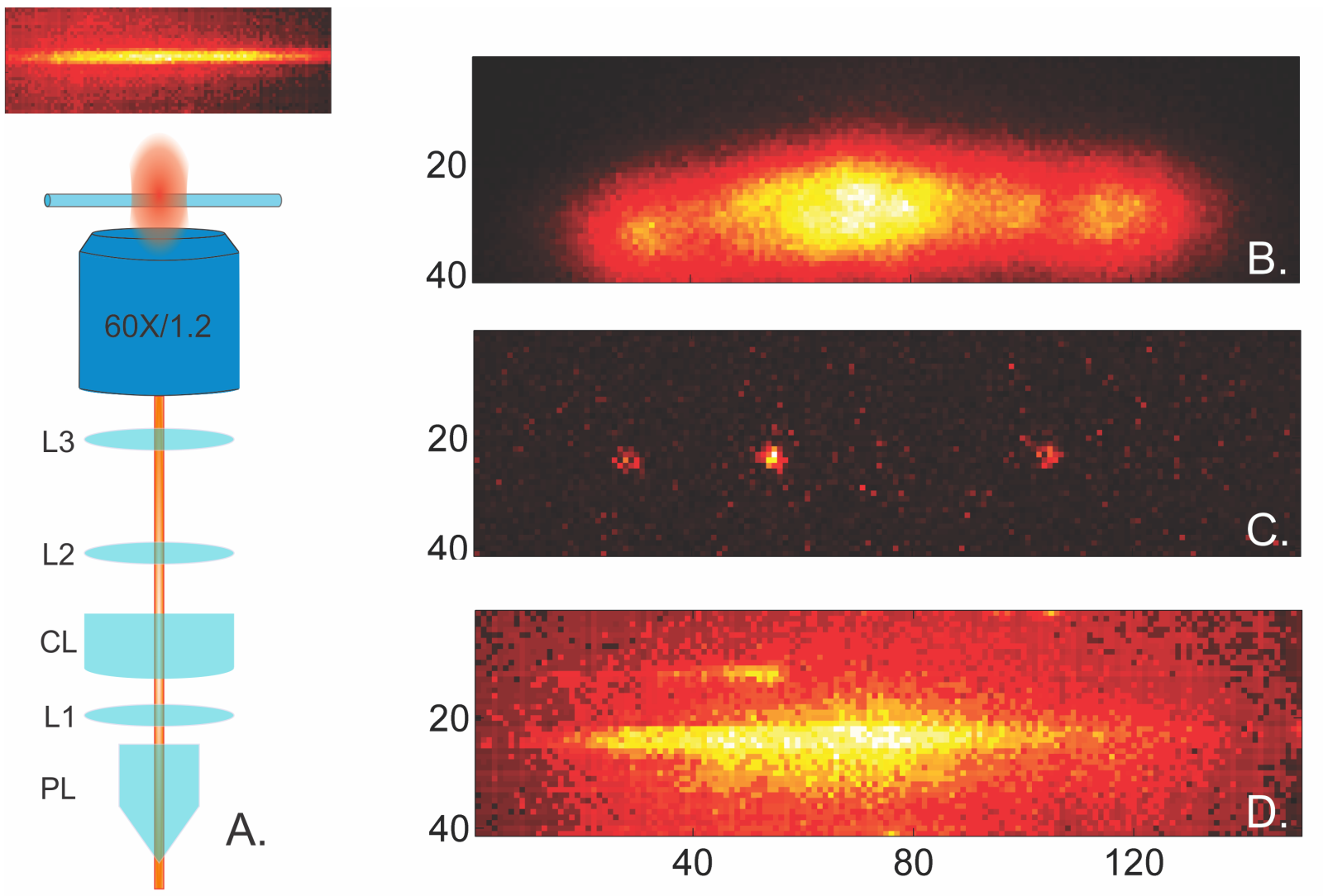

Figure 15: Single-molecule imaging inside 1D Nanochannels. (A) Schematic diagram of single channel excitation. The incident laser can be shaped as a rectangular flat-top beam by using combination of Cylindrical lens $(C L)$ and Powell lens (PL). The beam size is adjusted by three other Plano-convex Lens (L1, L2, L3). (B) A typical image frame for fluorescent molecules in free solution. (C) A typical image frame for fluorescent molecules in the Nanochannel. (D) Averaged image of multiple frames as in $(C)$.

the frame rate of EMCCD camera becomes the bottleneck for rapid imaging. Nonetheless, it has been shown that electrokinetic force can expediently regulate the molecular motion inside the Nanochannels ${ }^{70}$. In a diluted sample concentration, molecules imaged in a single frame are well isolated from each other. Due to its fast diffusion or transport inside the nanofluidic device, each single-molecule would be imaged as a smeared pattern under long exposure time. Figure 16 shows the different patterns generated under line-like (1D) and sheet-like (2D) confinements.

Molecular motion in a 1D Nanochannel leads to an ellipsoid shaped intensity pattern along the channel arrays in a camera image as shown in Figure 16 left. It is in distinct contrast 

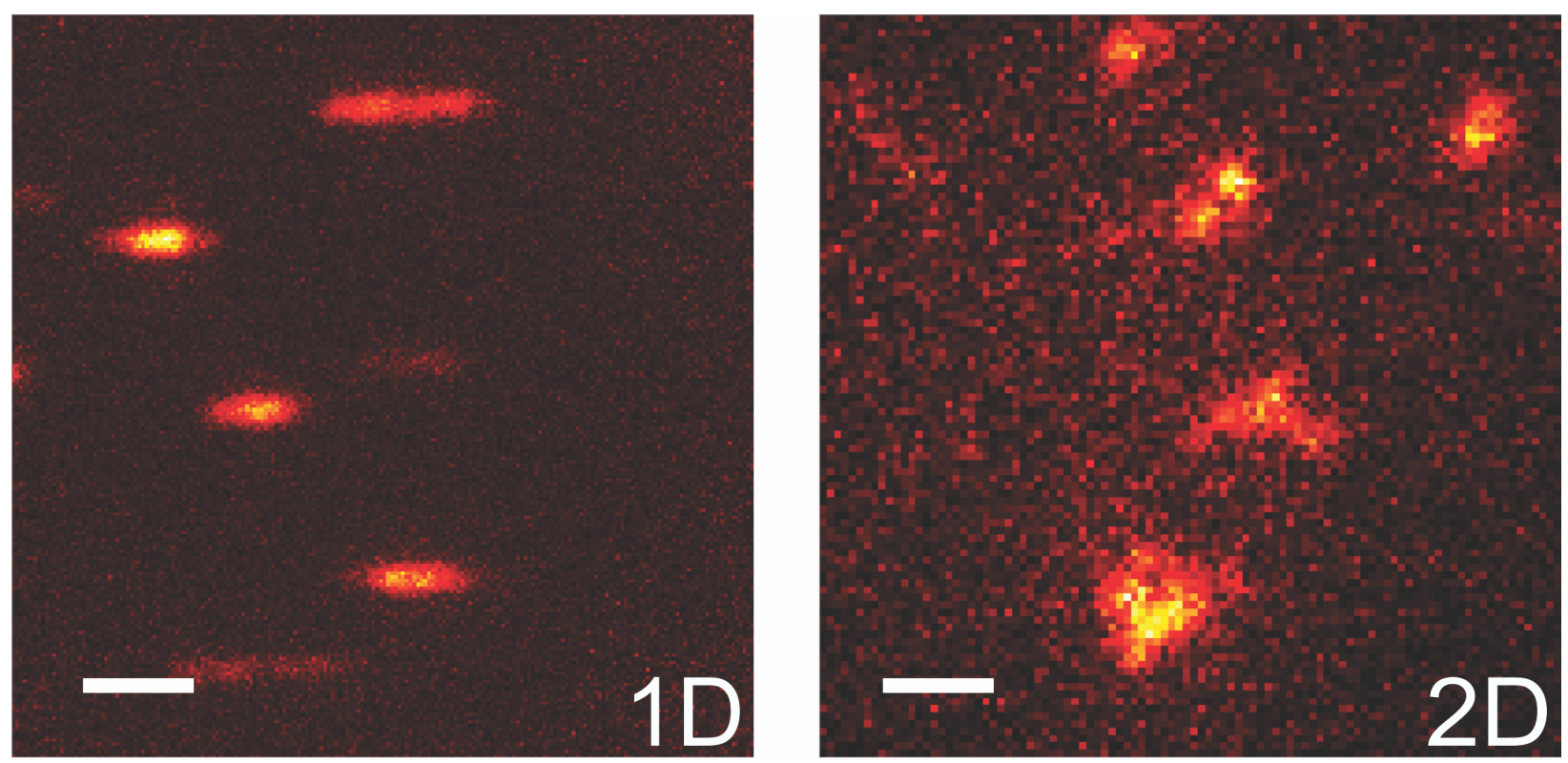

Figure 16: Comparison of single-molecule imaging with $1 D$ and $2 D$ confinements. The exposure time is $10 \mathrm{~ms}$. The scale bar is $2 \mu \mathrm{m}$.

to the pattern in 2D Nanochannel (Klearia) (Figure 16 right), where 2D molecular motion results in irregular trajectories. Within more confined 1D channels, if a controlled flow is applied, such that the molecular diffusion can be neglected, the intensity pattern is related to the moleule's brightness as a function of time. In other words, the intensity at each pixel position can be related to time within the frame exposure period. Therefore, each image can be regarded as a sum of multiple frames with shorter exposure times. In this way, one can investigate a molecule's brightness behavior with a higher temporal resolution.

One straightforward application of this method is to count molecules using photobleaching. Photobleaching of immobilized molecules has been employed on probing of the number of monomers per oligomer of protein assemblies ${ }^{[128}$ or membrane-bound proteins ${ }^{[16]}$. Such experiments require careful surface immobilization and any heterogeneity of the surface properties, such as the presence of local charges can deteriorate the performance of counting results. In contrast, the method described above allows one to measure the photobleaching steps on molecules that are transported along the channels, free from surface induced perturbations. Figure 17 shows the intensity pattern of Atto $647 \mathrm{~N}$ labeled a dsDNA in the 1D Nanochannel, acquired with a $10 \mathrm{~ms}$ exposure time. The photon counts from molecules are plotted against the direct of single Nanochannel in pixel base. One can clearly see the photobleach- 
ing steps along the molecule's trajectory. Typical two steps and one step photobleaching can be observed as shown in bottom panels of Figure 17. Within the field of view on a single frame, intensity patterns from multiple molecular trajectories can be obtained in parallel in Nanochannels array. Thus, this method holds a great potential for high-throughput single molecule counting experiments. Furthermore, smFRET experiments can be performed by monitoring the molecular brightness changes in two color channels simultaneously. This will allow one to probe into the molecular dynamics of complex biomolecules without the requirement of surface tethering.

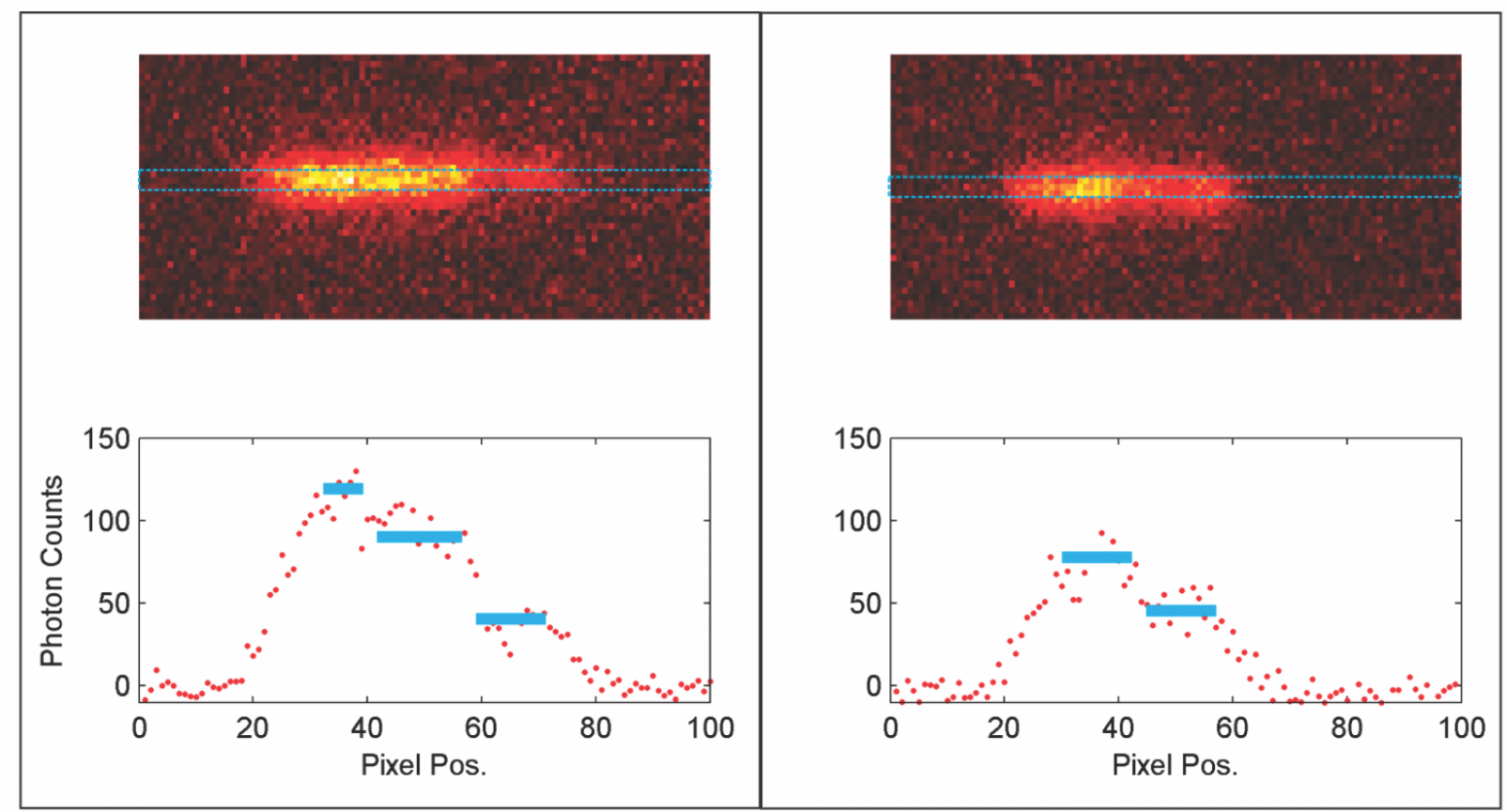

Figure 17: Single-molecule bleaching steps of Atto 647N labeled dsDNA detected in 1D Nanochannels. Intensity trace is plotted with respect to the pixel axis along the channel (dashed blue line). Examples of two steps (left) and one step (right) bleaching are demonstrated respectively. 


\subsection{Single-molecule manipulation in 1D Nanochannels}

\subsubsection{Electrokinetic flow}

Since the molecular motion is confined by the specific structure of the fluidic device, the manipulation of single molecules can be facilitated by regulating flow transport. The active flow control in a nanofluidic device can be realized through pressure pumping or more efficiently electrokinetic force. Electrokinetic flow is preferred because it is capable of producing a nearly uniform 'plug' profile that reduces sample dispersion. In contrast, pressure-driven flow produces a parabolic velocity distribution, leading different fluid layers to move with various velocities 30 . Additionally, the average velocity by pressure-driven scales with the square of the channel diameter, making it less favorable to work with submicrometer-sized channels 25 . Hence, electrokinetic force has been chosen to generate the active flow and manipulate single-molecule motion inside 1D Nanochannels.

Typically, electrokinetic flow is activated due to electroosmosis and electrophoresis. Electroosmosis describes the flow of liquid solution about a charged surface. More specifically, it is the so-called electrical double layer (EBL) moving along the channel wall under an external electrical field, which eventually drags the movement of the overall bulk solution. The EBL is formed by the immobile Stern layer and the mobile Gouy-Chapman layer, resulting from the interaction between electrolyte and charged surface ${ }^{2}$. Debye length is used to characterize the scale of EBL, which gives:

$$
\kappa^{-1}(n m)=\frac{0.307}{\sqrt{I(M)}}
$$

where $I$ is the ionic strength written with a unit of $M o l / L . \kappa^{-1}$ is less than $10 \mathrm{~nm}$ for ionic strength higher than $1 \mathrm{mM}$. Therefore, under a typical physiological condition, the EBL thickness is much smaller than the confinement size and a relative uniform flow velocity could be assumed along the optical axis direction in the solution. The simplified form of Navier-Stokes equation can be applied to calculate the electroosmotic mobility, and the relation is:

$$
\mu_{e o}=\frac{\varepsilon \zeta_{e o}}{\mu}
$$

Here, $\varepsilon$ is the dielectric constant and $\zeta_{e o}$ is the zeta potential that equals to the electric potential at one Debye-length away from the charged surface. $\mu$ is the viscosity of the solution. 
Electrophoresis, on the other hand, describes the movement of a charged molecule or particle about its surroundings under an external electric field. Assuming that a spherical particle with radius $R$ and surface charge $q$ is moving in a liquid with viscosity $\mu$, the steady-state will reach to:

$$
q E=6 \pi \mu R \nu_{e p}
$$

Here, the right-hide-side describes the Stokes drag for a particle velocity of $\nu_{e p}$. As the electrophoretic velocity $\nu_{e p}$ is proportional to applied electric field $E$, this proportionality factor is then named as the electrophoretic mobility and is written as:

$$
\mu_{e p}=\frac{\nu_{e p}}{E}=\frac{q}{6 \pi \mu R}
$$

Another EBL also surrounds charged molecules such as DNA or proteins in solution. The surface charge density of a molecule/particle can be estimated from its zeta potential $\zeta_{e p}$ and described as:

$$
\rho=\frac{\varepsilon \zeta_{e p}}{R}\left(1+\frac{R}{\lambda_{d}}\right)
$$

Here $\lambda_{d}$ is the Debye length and is much larger than the size of small molecule ${ }^{2}$. Thus, from Eq. 32, the electrophoretic mobility can be further estimated as:

$$
\mu_{e p}=\frac{4 \pi R^{2} \varepsilon \zeta_{e p} / R}{6 \pi \mu R}=\frac{\varepsilon \zeta_{e p}}{3 \mu}
$$

It should be noted here that the derivation didn't take into account of the distorted ions distribution that would generate a 'retardation force' to move the molecule/particle in the opposite direction. Therefore, an additional correction factor is needed to model the electrophoretic mobility in Eq. 34.

As shown from Figure 18, the electrokinetic transport in the channel is a combined effect of electroosmosis(EO) and electrophoresis(EP) for charged molecules. In practice, the exact measurements of EO and EP mobility in the channel are very challenging due to the difficulty of characterizing surface charges [117. Nevertheless, for the experiments in this dissertation, when a reasonable preliminary experimental condition is established, the molecular motion can be monitored by real-time imaging. The elecltrokinetic flow is provided by applying adjustable external voltage with specific ions strength that enables a flexible and adaptable manipulation of single-molecule inside the channels. 


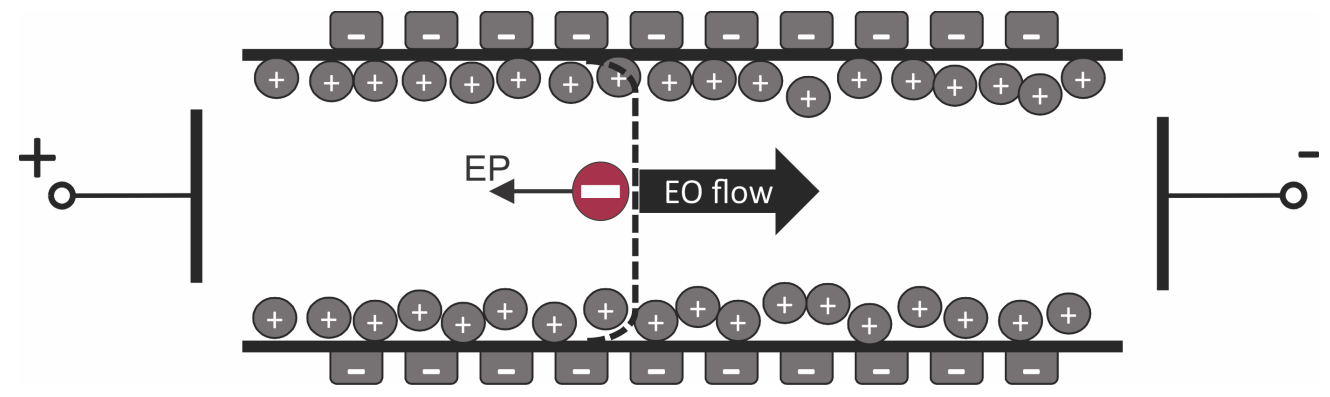

Figure 18: The electrokinetic response of molecule in solution combines the both effects of electrophoresis (EP) and electroosmosis (EO).

\subsubsection{Single-molecule recycling and trapping}

One elegant application of the electrokinetic flow in single-molecule studies is ABEL trap, developed by A. Cohen and W.E. Moerner 22] 23] 20]. In their device of a 2D nanoconfinement, fast single-molecule detection combined with active flow control is capable of compensating for Brownian diffusion, trapping the molecule on almost fixed position. Lately, J. F. Lesoine and L. Novotny applied a similar concept but simplified the system by utilizing a nanofluidic channel with a cross-section of $\sim 600 \mathrm{~nm} \times 400 \mathrm{~nm}$, which can confine the molecular motion only to a line-shaped 1D space ${ }^{70}$. Two electrodes were placed on both sides of the channel, and the direction of electrokinetic flow was changed alternatively depending on the detection events of single molecules passing through the laser focus. In this way each individual molecule was shuffled back-and-forth through the detection volume until photobleaching. This is termed single-molecule recycling.

Single-molecule recycling allows for measuring photophysical properties of the fluorescent label on each individual molecule. In this way, conformational dynamics, and other physical parameters such as charge/mass ratio can be measured on the same single molecule in solution. A severe limitation of this method is that one cannot distinguish the situation where multiple molecules enter the focus simultaneously.

Imaging-based approach, combined with 1D Nanochannel, enables direct tracking of single molecules (see in Section 3.2). As the sheet-like detection renders a much longer observation window for each molecule, the trapping idea is implemented here to provide a simpler and more flexible single-molecule manipulation system.

Two platinum electrodes are placed at each end of 1D Nanochannels to exert external voltage. 


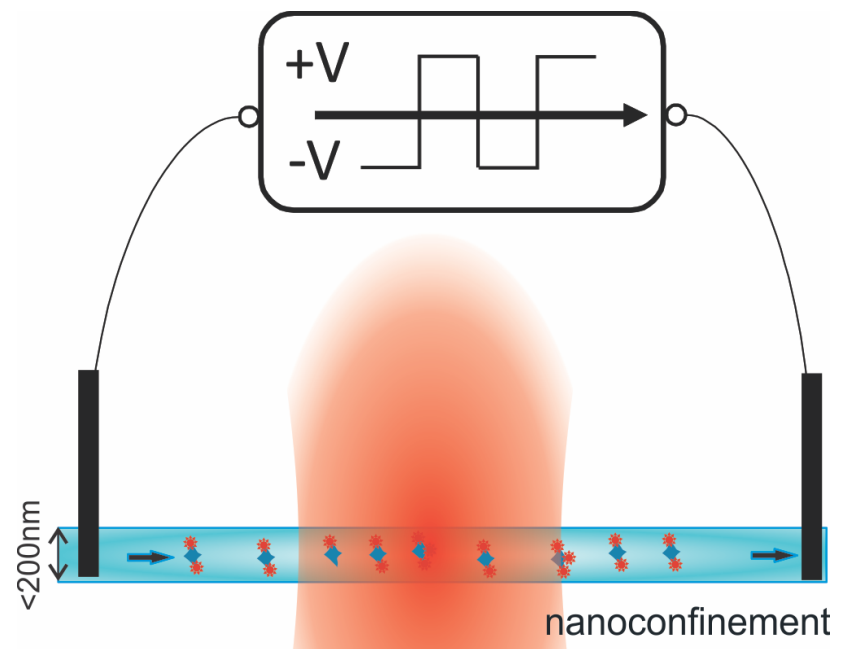

Figure 19: Schematic diagram of single-molecule manipulation by electrokinetic flow.

Atto $647 \mathrm{~N}$ labeled dsDNA (42bp) molecules are transported in the channels by electrokinetic flow. Long residence time of molecules in the detection volume enables monitoring of molecular motion response to applied voltage in real-time.

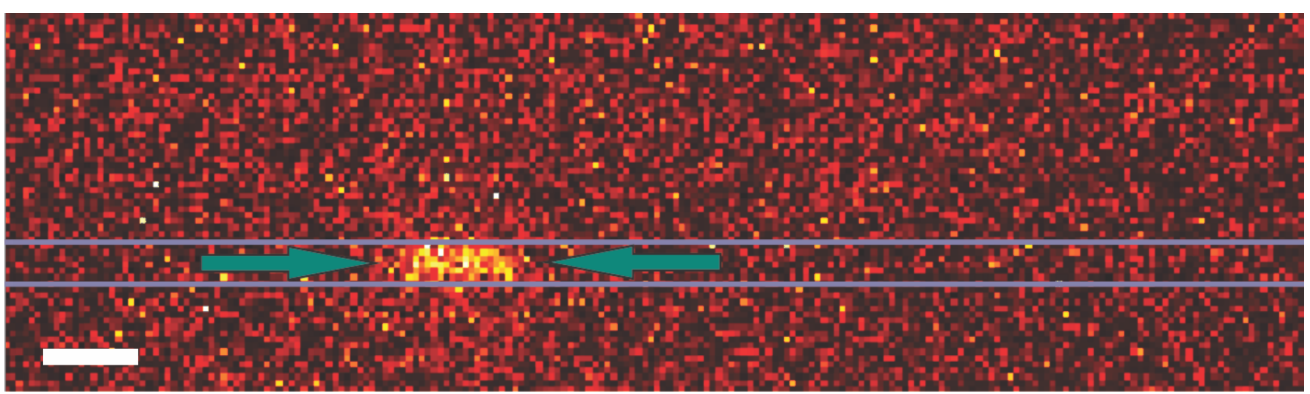

Figure 20: Real-time image of single-molecule motion in 1D Nanochannel under the voltage of alternating aurrent $(A C)$. The scale bar is $1 \mu \mathrm{m}$.

Interestingly, single-molecule recycling and trapping can be realized by adjusting the strength and alternative frequency of the voltage. A square wave $5 \mathrm{~V} \mathrm{AC}$ voltage is applied to alternatively change the flow direction.

If applying a low $0.5 \mathrm{~Hz}$ frequency, recycling is realized as molecules are repeatedly moving in and out from the detection field of view. On the accumulative fluorescent intensity plot, the events appear as the periodic peaks, captured at a frame period of $20 \mathrm{~ms}$. As shown in 


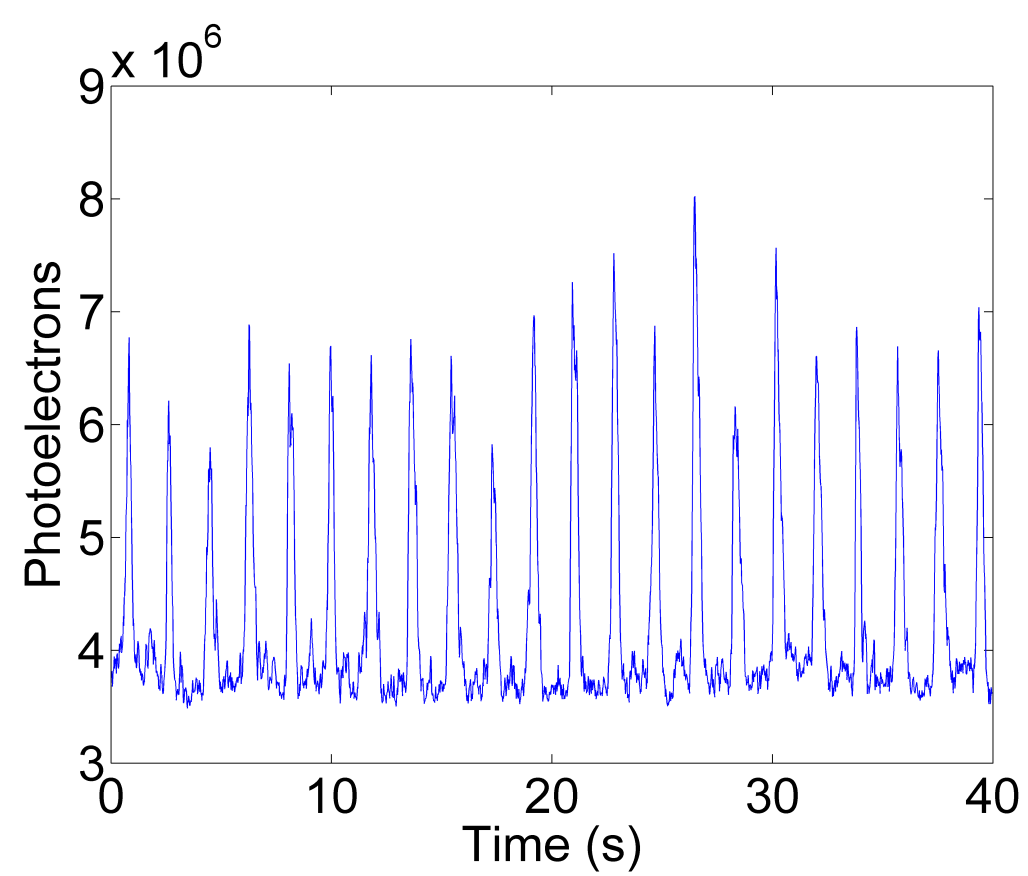

Figure 21: Single-molecule Recycling. Each data point of the time traces is the integration of the photoelectrons from an overall single image frame, with time bin of $20 \mathrm{~ms}$.

Figure 21, the intensity is the directly photoelectrons integration of each single frame and the acquisition of 40 seconds is demonstrated. The intensity peaks are fluctuating in tempo with the applied voltage frequency.

If the frequency increase to be fast enough to maintain the residence of a molecule in the detection field of view, recycling mode is switched into trapping mode. Therefore, the individual molecule can be kept visualized and investigated till it photobleaches. Figure 22 demonstrates the intensity time trace under a $20 \mathrm{~Hz} \mathrm{AC}$ voltage driven, where a zoom-in figure shows a total trapping time of $\sim 3$ seconds for an individual molecule inside the channel. Figure 23 is the averaged images of the consecutive frames recorded in this trapping period, and it manifests the residence location of this individual molecule under the electrokinetic flow.

Compared to the confocal-based methods, this method enables a continuous image recording of single-molecule motion in solution. It is also immune to multiple molecules problem. Since the feedback control is no longer obligatory here, it is much easier to be implemented. Moreover, by using multiple electrode pairs in a Nanochannel, more than one molecule can be controlled and monitored in parallel. It provides a suitable platform to study single- 

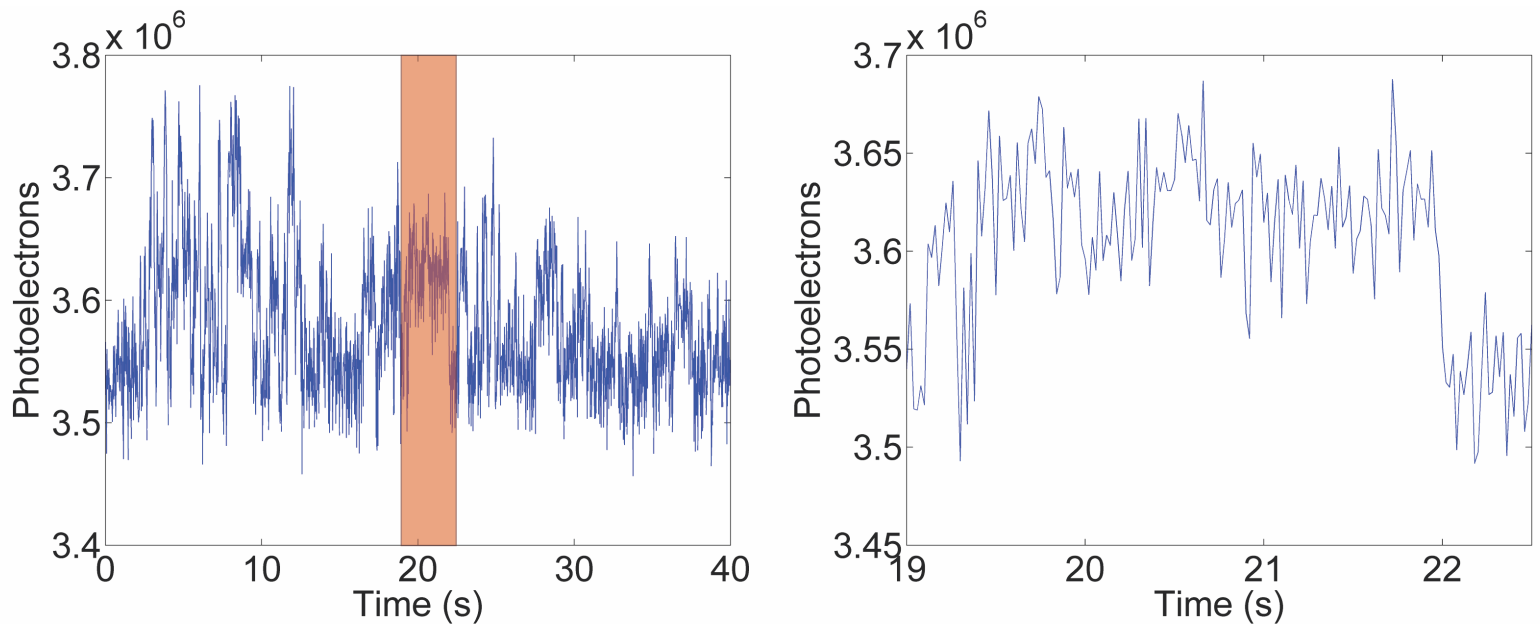

Figure 22: Single-molecule Trapping. On the right side is a zoom-in plot of time trace in the red shadow highlighted part for the left figure. It shows a trapping event of molecule for around 3 seconds till its photobleaching.

molecule conformational dynamics and molecular interactions in extended timescale under controlled condition.

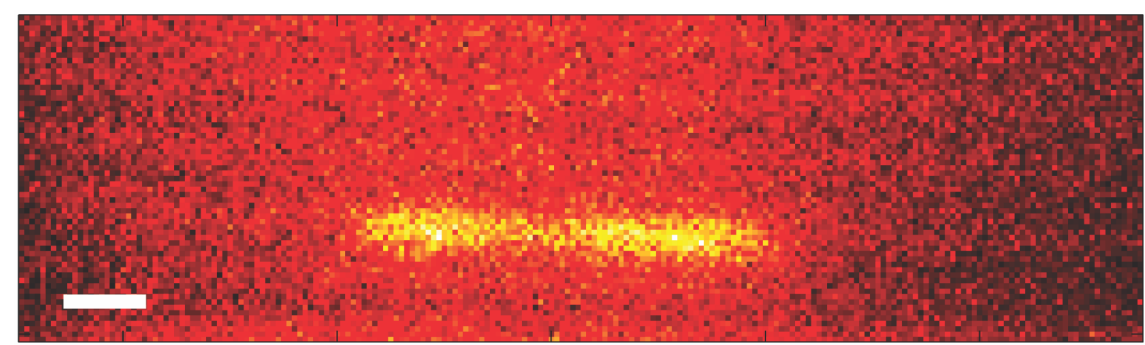

Figure 23: Residence position of the trapped molecule. The profile demonstrates the trapping region for a single molecule under a $20 \mathrm{~Hz} A C$ voltage. The scale bar is $1 \mu \mathrm{m}$. 
48 3. Single-molecule DeteCtion And Manipulation in NANOFLUidic ChanNels 


\section{Imaging-based photon counting histogram (iPCH) for measuring the distribution of molecular mixtures}

Nanofluidic devices greatly facilitate the detection and manipulation of single molecules $\frac{86}{\text {. }}$ In Chapter 3, I've shown using nanofluidic devices for single-molecule spectroscopy and imaging, which enables the study of various single-molecule properties. One of the key parameters of a fluorophore is its molecular brightness, which is proportional to its absorption cross-section times its fluorescence quantum yield. A method that could accurately and quantitatively determine the brightness on a single-molecule level would make it possible to determine the stoichiometry of a molecular complex.

Molecular brightness is typically determined from the number of detected photons in a given period of time. When immobilized molecules are measured, the heterogeneity of their local environments causes high variations of measured values 110 . Moreover, surface tethering would often disrupt the molecular behavior $\stackrel{69}{ }$, making the observed molecular properties different from that of a molecule in biologically relevant environment. For these reasons, accurate brightness analysis needs to be conducted in immobilization-free conditions, i.e. in free solution. Different suggestions to perform such measurements exist, but suffer from unsatisfactory performance or limited resolving power 15 [16 [62. A confocal-based detection configuration is usually applied to improve the SNR, using a single photon detector to record the intensity time trace of single molecules. However, the drawback to brightness analysis is that, due to Brownian motion, each diffusing molecule transits the detection volume $(\sim 1$ fL) along an irregular trajectory. Every molecule experiences different illumination and generates a non-uniform fluorescence intensity time trace. One solution to this problem is to devise a dynamic experimental scheme to keep the moving molecule under a constant excitation/detection condition, which requires single-molecule tracking that is extremely challenging when working with small molecules of only several nanometers in size $\frac{53}{4}$ A more practical method is to employ active transport which helps ensuring an identical excitation/detection process. R. Keller and colleagues exploited an optimized flow cell to align a sheath flow that guides molecules across the stationary detection uniformly $[99[37]$ 38] The follow-up method of Cylindrical Illumination Confocal Spectroscopy (CICS) used a similar idea but optimized the detection volume by creating a sheet-like illumination ${ }^{74}$. However, 
confocal-based single-molecule BSD analysis is only able to work on one molecule at a time, which results in a low-throughput. The interpretation of fluorescence bursts is also errorprone, as it requires the precise characterization of molecular transport and the detection volume. In practice, a $\mathrm{BSD}$ with small variance can be achieved only if a relatively high flow rate is applied, to alleviate the influence of diffusion. At the same time, this reduces the residence time in the detection volume, which limits the photon budget from each molecule. Nowadays, the advent of nanofluidics enables a different solution to this problem. As discussed in Chapter 3, instead of minimizing the optical detection volume, the high SNR study of single-molecule is conducted by reducing the space molecules are able to move in through nanoconfinement. By utilizing an EMCCD camera, the rather indirect intensity burst is replaced by straightforward imaging of individual molecules during their diffusion or flow transport through nanofluidic-channels. A full-glass-chips with channel heights of less than $200 \mathrm{~nm}$ is used to confine the molecular motion to the focal plane of the observing microscope. This provides a platform to precisely monitor and to manipulate individual molecules in solution. Experimental results have shown that high-quality images of freely diffusing single fluorophores can be recorded and their brightness can be determined with high accuracy. Furthermore, as the molecular motion is directly visible from the images, brightness analysis can be performed without influence from the irregular trajectories. Since multiple molecules can be detected in parallel, this system has a much higher throughput, detecting thousands of molecules in only several minutes.

\subsection{Stroboscopic imaging}

In the confocal-based method, single-molecule brightness information is extracted from the fluorescence burst of an intensity time trace and the resolving power is determined by the homogeneity of the BSD, Likewise, in the imaging-based scenario, single-molecule detection events are represented as characteristic fluorescence imaging patterns. Therefore, a desirable performance of the brightness analysis requires uniform single-molecule imaging patterns to be recorded. Due to the molecular motion, the variance of these imaging patterns increases with the duration of the exposure time (see the discussion in Section 4.4). 


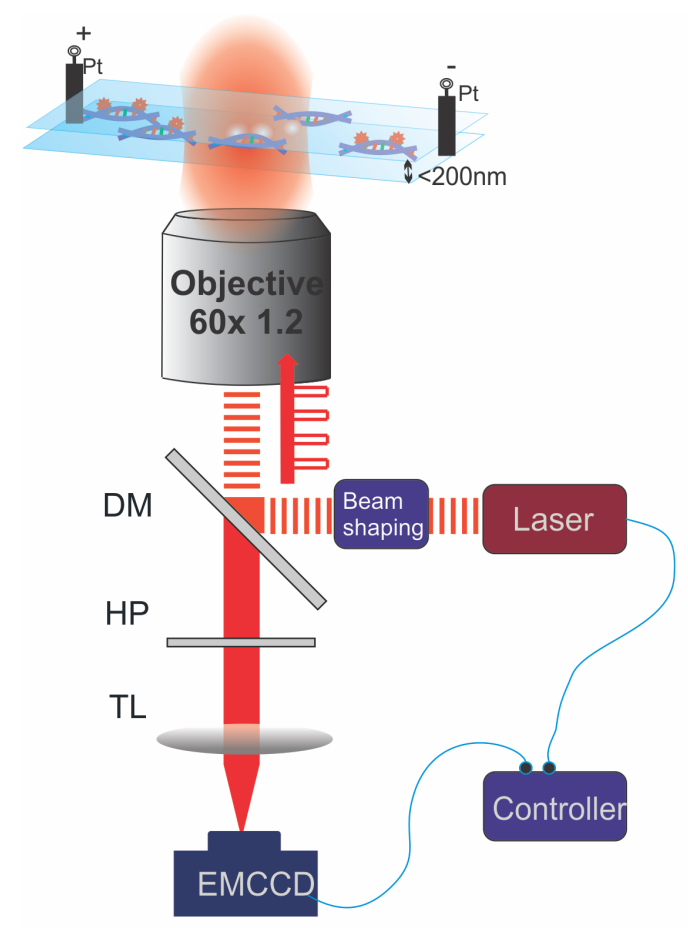

(a)

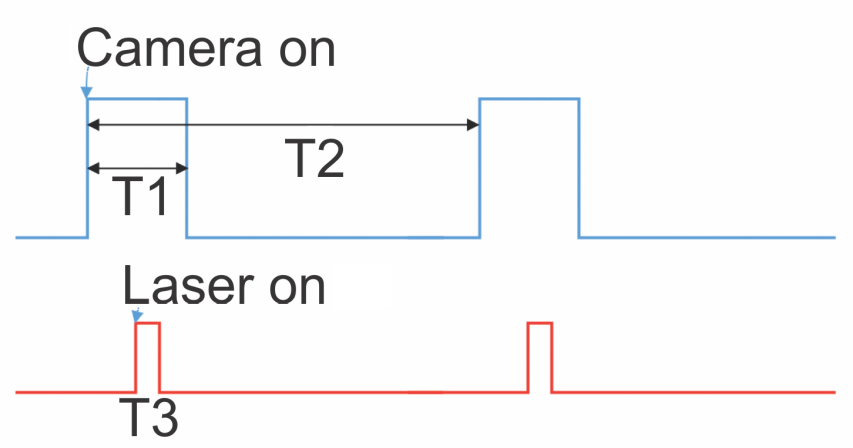

(b)

Figure 24: Stroboscopic imaging system. (A) Schematic diagram of imaging single-molecule flow in nanofluidic channels. Molecules are confined in a $1 D$ channel or $2 D$ plane, where the height is less than 200nm. Fluorescence signal is capture by passing through the optical components of dichroic mirror (DM), high-pass (HP) filter and tube lens (TL). (B) The synchronized work stream of camera imaging and laser excitation.

An EMCCD working in fast frame transfer mode can reach a frame rate of ca. $1 \mathrm{kHz}$. For a typical organic dye with a diffusion coefficient $D$ that has a value of $\sim 100 \mu \mathrm{m}^{2} / \mathrm{s}$, in the detection period $t$, the diffusion-based 2D mean squared displacement (MSD) is calculated as $4 D t$. Thus, in contrast to the detection PSF that is obtained from the image of the immobilized molecule, imaging patterns for diffusing molecules are very much smeared by this $\sim 0.4 \mu \mathrm{m}^{2}$ displacement. In this case, an even shorter detection time that not restricts to the camera frame rate is preferred. Meanwhile, the capability of identifying and localizing each molecule is connected with the number of recorded photons. With an average of 100 photons needed, the count rate should be larger than $10^{5}$ counts/s. It requires a high excitation power density, where photobleaching would be pronounced. For these reasons, a stroboscopic imaging system is introduced with multiple advantages. 
This is realized by synchronizing the laser illumination pulses with the continuous EMCCD camera recording. As shown in Figure 24 of the working stream, two TTL pulses with an identical period (T2) are generated from a Programmable Counter/Timer Devices (PCI6602, NI). T3 represents the illumination time of the laser pulse, which can be shorter than the minimum camera exposure time (a few milliseconds) that is usually limited by read-out speed. In stroboscopic excitation, the illumination time of each frame is regulated by the laser pulse length.

Typically, when the illumination time is short enough, molecules are regarded as stationary. In Figure 25, a comparison of images recorded by using different pulse lengths is demonstrated. The elongated ellipsoid shaped single-molecule intensity patterns extending along the channel arrays are visualized when using a long illumination time. Analyzing the patterns under various values of T3, information of molecular mobility e.g. diffusion coefficient can be estimated, which is discussed further in Section 4.4.

Experiments are conducted on a home-built epi-fluorescence microscope by using a waterimmersion objective (UPLSAPO $60 \times \mathrm{W}, 1.2$ N.A., Olympus) for both illumination and detection. The cleaned up laser beam (OBIS 637nm LX 140mW, Coherent, and MBL-III-473$100 \mathrm{~mW}$, CNI Laser) is coupled into a single mode fiber (P1-460AR-2, Thorlabs) and then collimated. A telescope lens pair ( $\mathrm{f} 1=50 \mathrm{~mm}, \mathrm{f} 2=75 \mathrm{~mm}$ ) is applied to adjust the beam size. Afterwards, it is focused on the back aperture plane of the objective by another lens ( $\mathrm{f}$ $=200 \mathrm{~mm}$ ), producing an illumination region of ca. $10 \mu \mathrm{m} \times 10 \mu \mathrm{m}$ at the focal plane. The illumination area is adjusted to guarantee a high excitation power density (from 10 to 100 $\mathrm{kW} / \mathrm{cm}^{2}$ ) with a relatively homogeneous excitation profile. The excitation beam is slightly tilted to be strayed away from the imaging path, helping to reduce the strong backscattering signal from the coverslip interface. A multi-band dichroic mirror (Di0-R405/488/561/635, Semrock) is applied to separate the illumination/detection channel before a long pass filter (F76-472 or F76-649, Semrock), which is also designed for further multi-color applications. Images or movies are captured by an iXon back-illuminated electron multiplying charge coupled device (EMCCD, iXon Ultra 897, Andor) working in frame transfer mode. Before the EMCCD, a 3.3× expansion lens pair (MAP1030100-A, Thorlabs) is placed before the tube lens $(\mathrm{f}=200 \mathrm{~mm})$. The corresponding pixel size of the image is $61 \mathrm{~nm}$. Pixel binning of $2 \times$ 2 is used during all the brightness characterization experiments. 

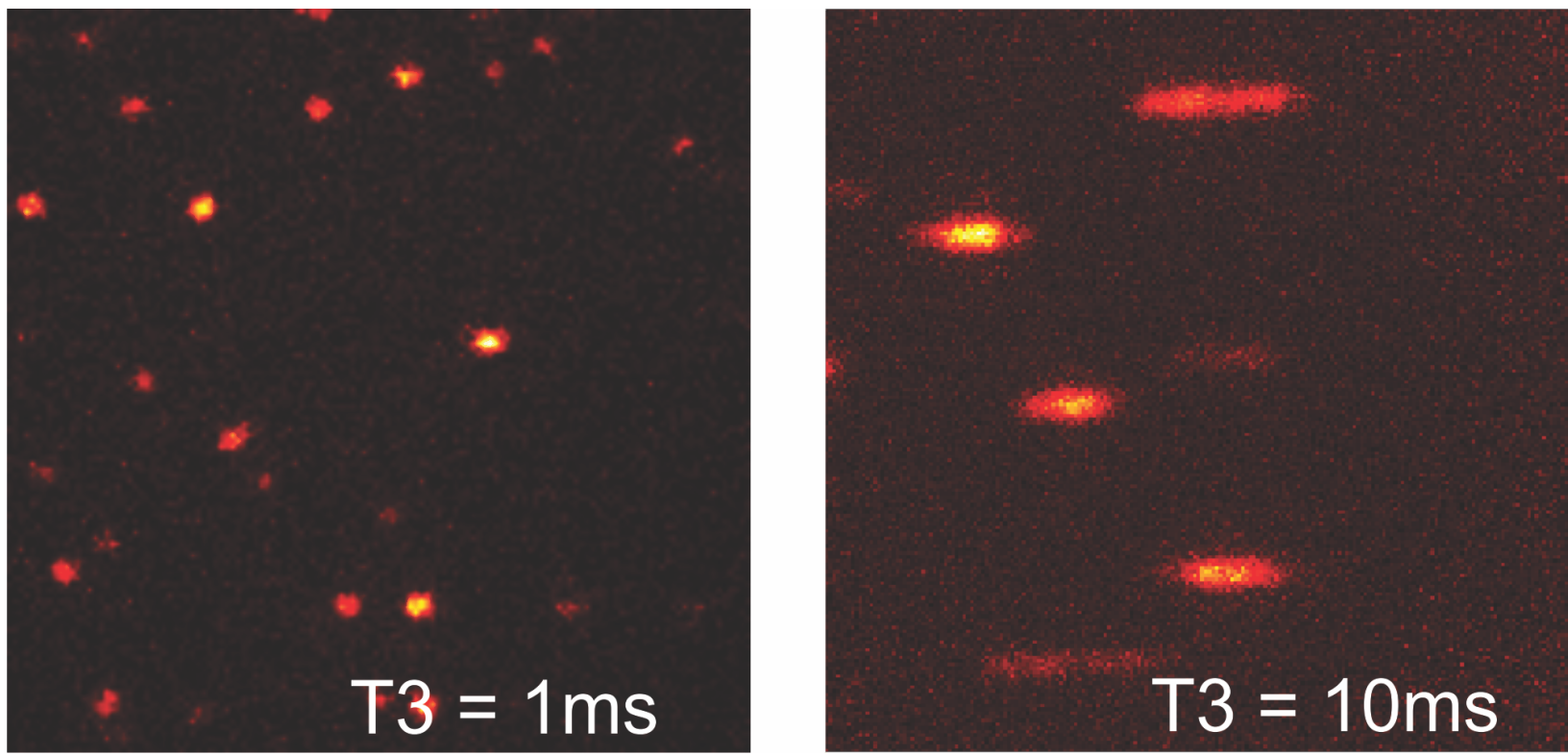

Figure 25: Comparison of stroboscopic images captured by using short (1 ms) and long (10 ms) pulse excitation. From imaging of Atto $647 \mathrm{~N}$ labeled DNA in $1 D$ nanochannels.

For brightness characterization, multiple image frames are recorded. Since obtained images are similar to immobilized single-molecule images, the analysis can be carried out using well-established single-molecule analysis toolboxes. As described in Figure 26, the absolute brightness of every single molecule can be determined from the movie by first localizing each molecule and fitting with a suitable model to get the intensity distribution or simply summing up the pixels in a neighborhood around its localized position, which is conducted using publicly available software 107 . The resulting analysis of thousands of molecules yields the brightness distribution, which is termed imaging-based photon counting histogram (iPCH). The brightness distributions presented are all calculated by integration of the pixel values around each estimated position, which a certain area defined as region of interest (ROI) pixels. Given that a larger ROI than the size of single-molecule image patterns is used for photon counting, non-biased brightness information can be achieved simply by subtraction of the properly estimated background signal.

To prove that an integration of pixel values in a $\mathrm{ROI}$ can precisely reveal the single-molecule brightness distribution, the $\mathrm{iPCH}$ analysis is also tested on simulation data.

Here, random walks of molecules diffusing in a 2D plane are used to generate the singlemolecule imaging patterns, corresponding to the situation of molecular motion in 2D nanochan- 


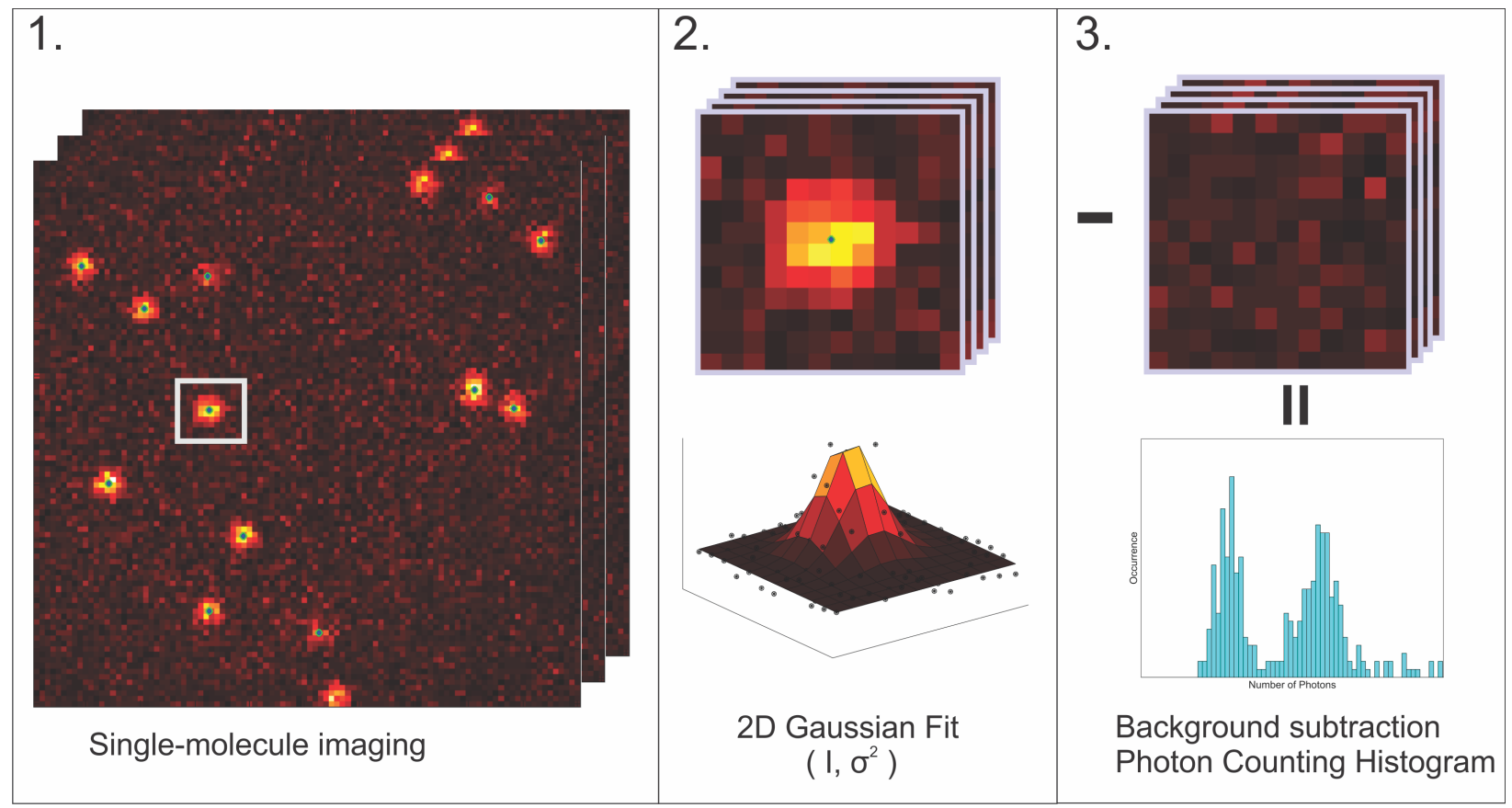

Figure 26: Illustration of iPCH method. (1) Multiframe images are recorded for moving molecules inside the nanofluidic channels. (2) Each localized molecule is fitted by a 2D Gaussian function. The ROI pixels are chosen based on the fitting results. (3) Brightness distribution is obtained by counting the photon numbers in each ROI, with a subtraction of background.

nels. At each simulation time steps of $10 \mu \mathrm{s}$, the mean displacement of molecule position is less than one simulated pixel size $(120 \mathrm{~nm})$. Thus, molecules are regarded as stationary. A certain amount of photons from individual molecules are spreading on a pixelated imaging plane, with a range described by a 2D Gaussian detection PSF (here with standard deviation set to $180 \mathrm{~nm})$. In average 400 photons coming from a single-molecule are simulated in an illumination time of $1 \mathrm{~ms}$, exhibiting distinct emission patterns depending on various diffusion coefficients. According to real experimental condition, a background is set as 3 photons/pixel.

, For brightness characterization, two different sizes of ROI centered around the localized positions of molecules are compared in Figure 27. In the situation of $9 \times 9$ pixel window size, single-molecule brightness is underestimated for fast diffusion coefficient of $100 \mu \mathrm{m}^{2} / \mathrm{s}$ and almost identical for the two slower diffusion cases. The reason is that photons spread over a broader range cannot be covered by this window size for fast diffusing molecules. In contrast, when a relatively larger ROI of $15 \times 15$ pixel window is applied, iPCH analysis 
shows identical performance for all three cases, even though the diffusion coefficients are differing by two orders. Thus, using iPCH to measure the brightness distribution is free from molecular motion induced heterogeneity, which must be strictly controlled in all the confocal-based methods.
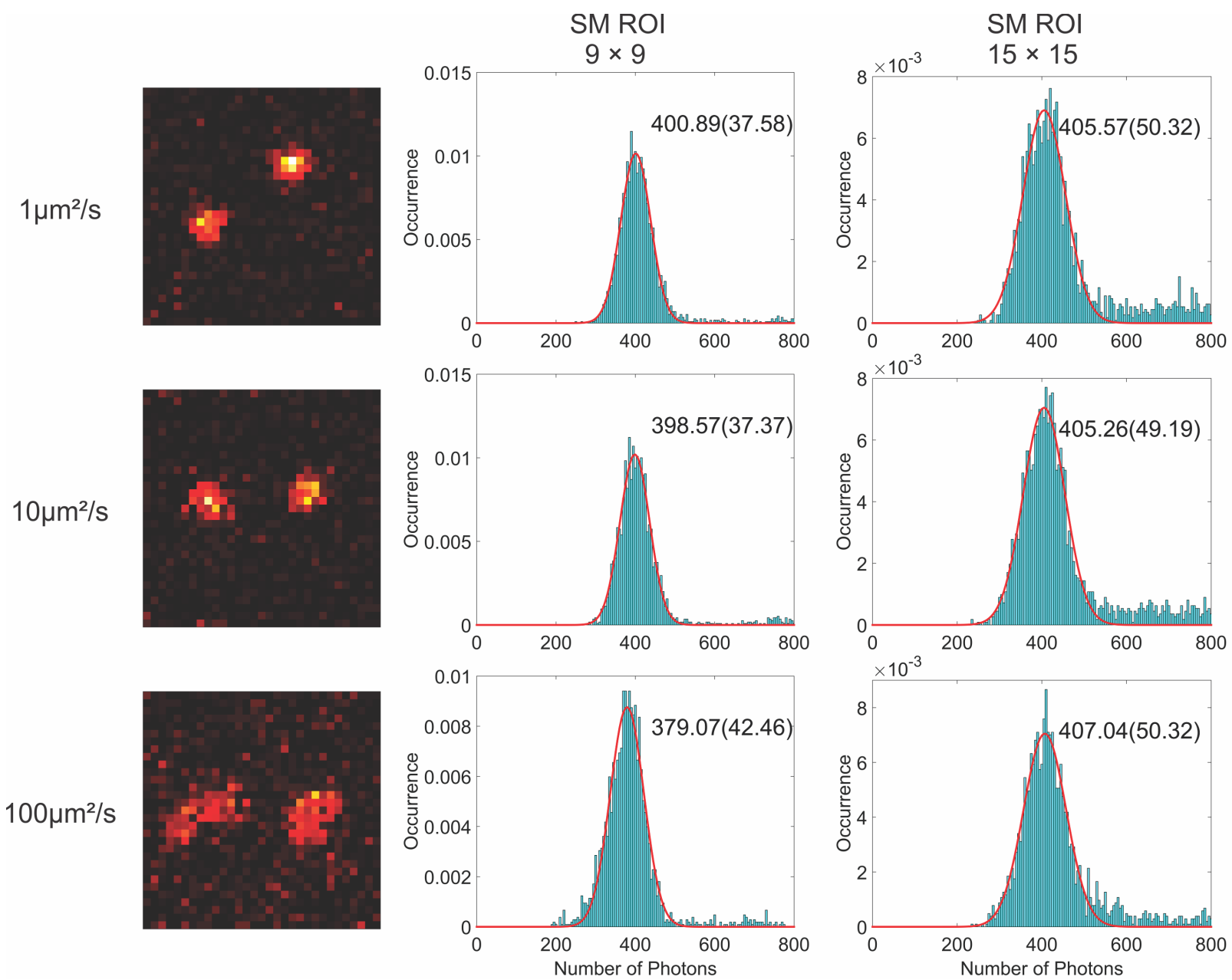

Figure 27: Test brightness characterizaiton ability by simulation data. Typical single-molecule imaging patterns for three different diffusion coefficients are listed. The corresponding iPCH analysis using two different ROI window size is given. All histograms are fitted by a single Gaussian model, where the mean values and standard deviations (in brackets) are stated.

Noticeably, when the big ROI window size is used, brightness distributions are broadened due to the contribution of more background signal included (see the discussion in next Section). Fortunately, as the background can be estimated from the overall image stack, it can be corrected. Moreover, big ROI window size may also include the photons emitted by the 
nearby molecules, which result in a tail of the brightness histogram, as seen from the $15 \times$ 15 ROI case in Figure 27. This introduces additional uncertainty and needs to be carefully considered especially when multiple molecular species are investigated. In practice, for fast moving molecules, a low sample concentration is preferred in order to reduce this crosstalk effect.

Furthermore, since a relative high excitation power density is required for gathering enough photons from the individual molecule, photobleaching would deteriorate the accurate brightness analysis. Using stroboscopic illumination and active flow control, a proper choice of T2 to a value of several tens of milliseconds helps to guarantee that each frame records new molecules. It provides considerable statistics from thousands of molecule in minutes. In combination with a short stroboscopic excitation pulse, the impact of photobleaching is alleviated. In addition, the pulse excitation also reduces the heat accumulation on optical components that is otherwise tend to be damaged under high power continuous wave excitation.

\subsection{Precise brightness characterization}

The resolving power of the $\mathrm{iPCH}$ method is determined by the quality of the obtained brightness distribution. The experimental parameters, e.g. laser-induced background signal, detector noise, emission properties of single-molecule and its interaction with the nanofluidic device, would all potentially affect the obtained histogram. Hence, a thorough investigation is performed to check the accuracy of iPCH method.

\subsubsection{Shot noise}

The brightness characterization is conducted by accumulating signal from a light source in a defined period. Here, single-molecule fluorescent emission is sensed as a stream of individual photons that hit onto the detector.

Under a constant excitation condition, the photon emission from single fluorophores follows 
Poissonian statistics. Therefore, given that the average photon counts per time intervals are $\lambda$, the shot-noise-limited photon counting number $k$ is distributed as:

$$
p(k ; \lambda)=\frac{\lambda^{k}}{k !} e^{-\lambda}
$$

Here the mean value $\lambda$ is also its variance. The sum of multiple random variables undergoing the same physical process will still follow the Poisson distribution, and the variances and mean values are simply additive.

\subsubsection{Background}

In nanoconfinement, background fluorescence from out-of-focus molecules is eliminated. The readout and dark current noise from the EMCCD is negligible when EM gain is applied. Thus, the laser excitation induced background signal $b$ (e.g. scattering, autofluorescence) is the dominant noise source in the measurement. Background photons are indistinguishable from the photons emitted by the molecules and can be regarded as an independent process that adds variance to the final brightness distribution.

\subsubsection{EMCCD effect}

The EMCCD camera with its superior sensitivity is especially suitable for applications where photon budget is limited. However, the electron multiplication process introduces an additional multiplicative noise, which results in additional variation in the overall number of signal counts. This uncertainty increases the shot noise based variance by a factor of two. A detailed theoretical description and modeling can be found in ref. [54].

The detection property of our EMCCD is characterized by measuring the light source backscattering from a constant blank plate. Here, the sets up of the system is identical to the experiments with fluorescent samples $(637 \mathrm{~nm}, 1 \mathrm{~ms}$ illumination time, $5 \mathrm{MHz}$ read out), except the emission filter is removed to ensure that the camera can detect more signal from the laser. The peak power which reaches the EMCCD is adjusted to the same level as the fluorescence molecules. A $5 \times 5$ pixel window in each frame is used to represent the 
single-molecule detection event and to integrate the photon counts, which is subsequently demonstrated as the iPCH distribution from 1000 image frames. As shown in Figure 28, the brightness distribution obtained here has a variance that is 2.05 times of the mean value. This is in excellent agreement with the camera modeling. This measurement also proves the stability of pulsed laser excitation, which won’t bring extra noise.

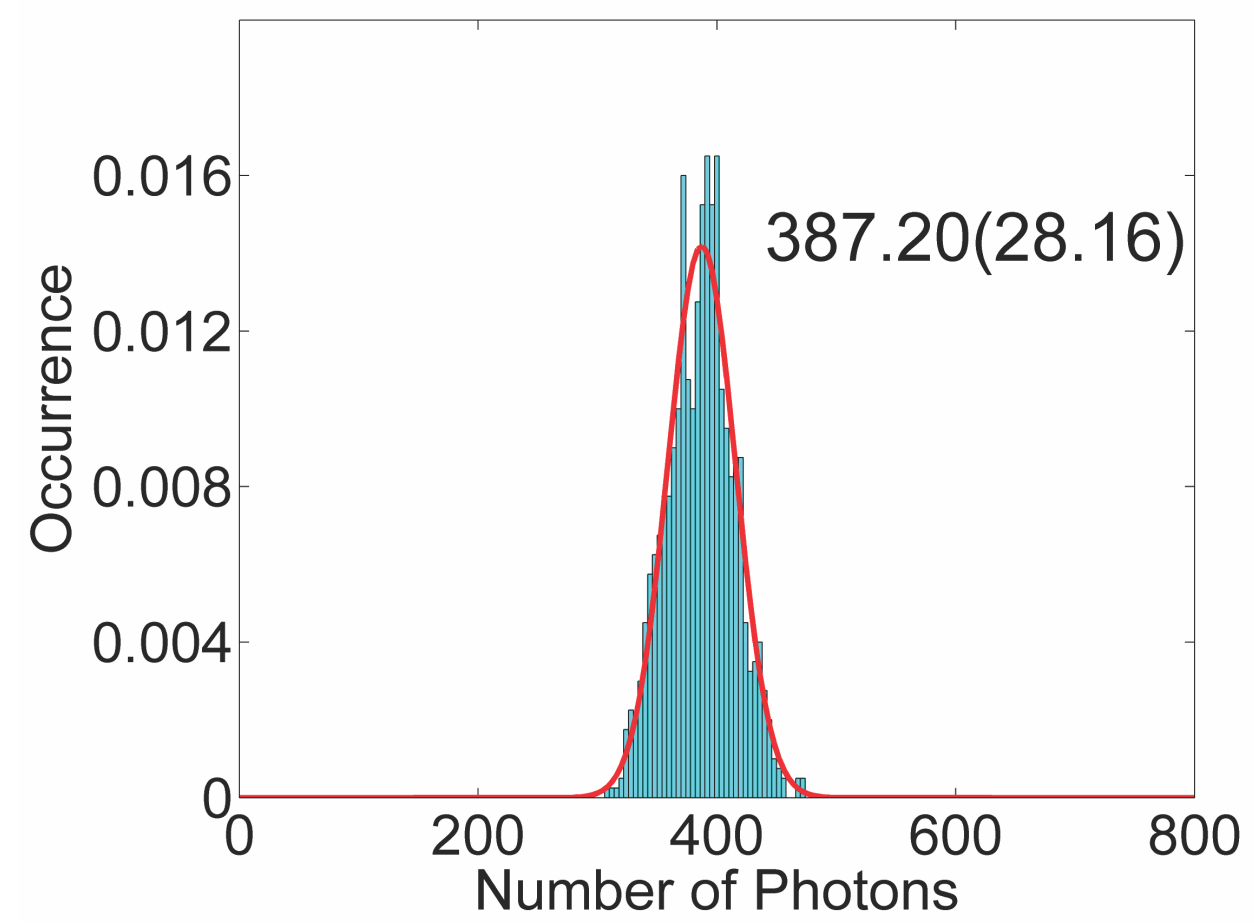

Figure 28: Illustration of the EMCCD effect. Here, iPCH analysis of backscattering from laser excitation is performed. The standard deviation (in brackets) obtained is 1.43 times larger than the shot-noise-limited distribution.

Consequently, the added-up detected signal variance with EM gain applied is simply written as:

$$
\operatorname{var}_{d e t}(\lambda, b)=2 \cdot(\lambda+b)
$$

\subsubsection{Excitation profile}

Excitation geometry is one of the essential experimental conditions in all presented singlemolecule brightness analysis methods. In confocal-based scenarios, the varying detection 
efficiency of irregular molecule trajectories in the excitation focus brings additional variance, making the brightness distribution much broader.
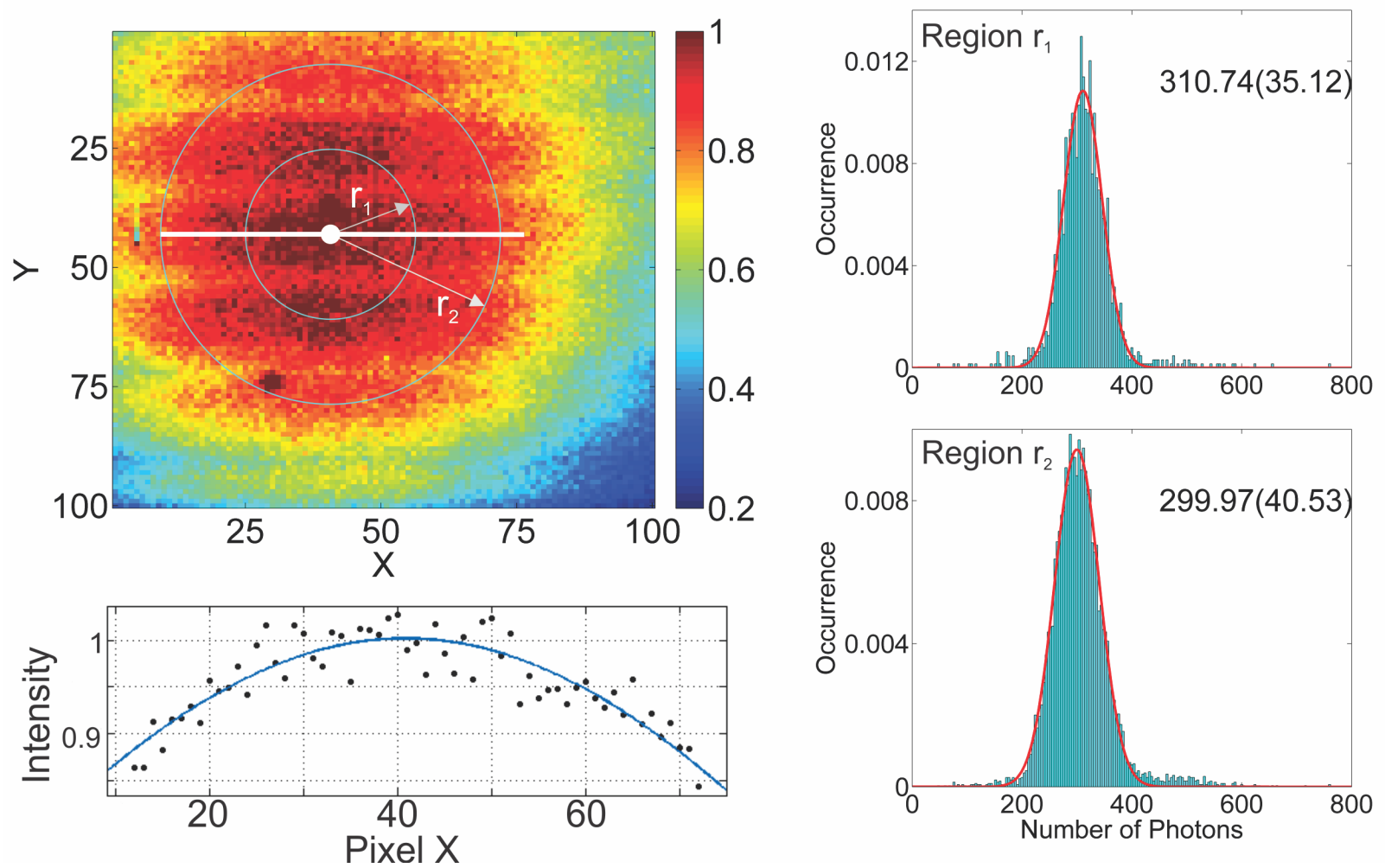

Figure 29: Characterization of excitation profile. A Gaussian-shaped excitation profile is shown, and iPCH analysis for Cy5 molecules under excitation of two different radii cutoffs is compared.

As a result of the large sheet-like detection volume in the imaging-based configuration, each single-molecule can undergo a more uniform excitation. Moverover, excitation conditions single molecules have been experienced are visible in each image frame. Since the excitation profile can be obtained from the average of all image frames, the brightness distribution can be further corrected. In Figure 29, measurement for Cy5 in 1D Nanochannels has been performed and the frame-averaged image of the whole image stack shows a slightly inhomogeneous excitation profile. As the fluorescence signal comes from the molecules distributed in the channel array, the profile is exhibited as a stripe pattern. The beam profile is Gaussian-shaped, as illustrated from the normalized intensity plotting along the central pixels (white line). The iPCH analysis of molecules localized in the two circular regions (15 and 30 pixels from the center) is then compared. As it shown in the 30 pixels case, a 
non-negligible increased variance is introduced when the excitation strength varies strongly between molecules. Fortunately, in a small circular region (e.g. 15 pixels), a homogeneous excitation can be assumed.

\subsubsection{Photophysics}

Photophysical properties of fluorophores also play a significant role in brightness estimation. The feature of short stroboscopic illumination can help lower the influence of photobleaching. However, the faster photoblinking effect cannot be ruled out.

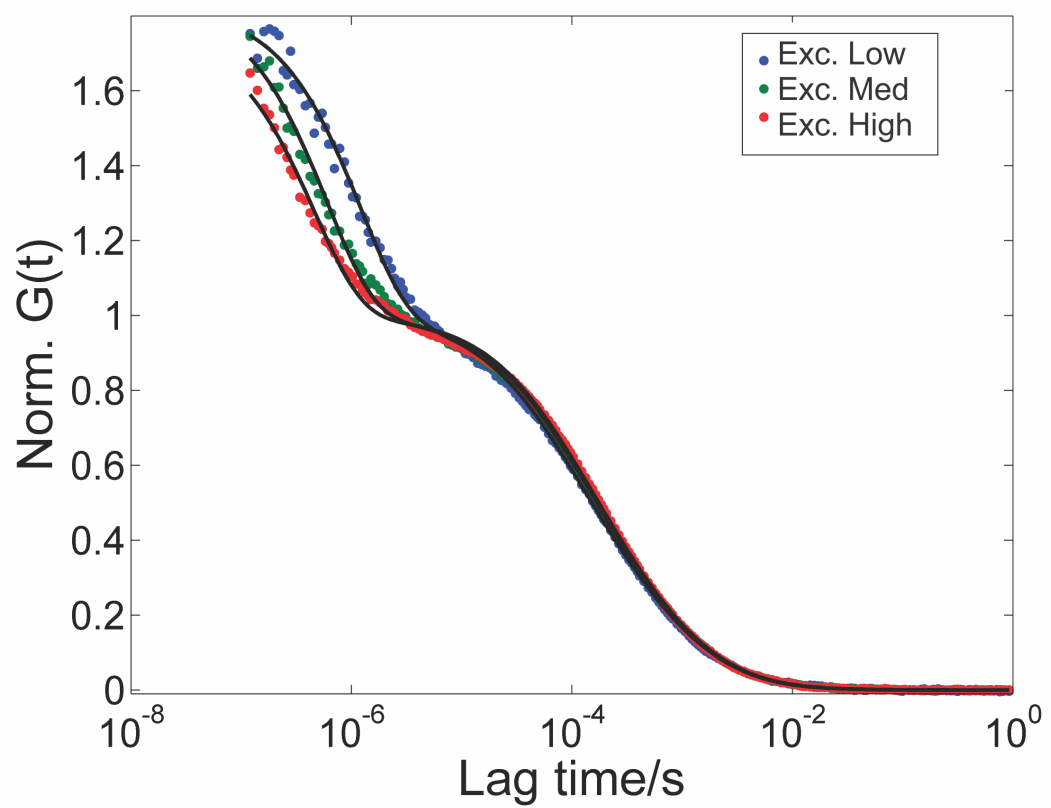

Figure 30: Characterization of blinking dynamics in free solution. FCS measurements of Cy5 in free solution are performed under different excitation power density. The blinking on/off dynamic rates are extracted from the fitting based on the model in Eq. 37.

A simple model of photoblinking assumes reversible transitions between two possible states with on-state lifetime $\tau_{o n}$ and off-state lifetime $\tau_{o f f}$. Interestingly, a comprehensive theoretically modeling proves that the blinking dynamics can introduce substantial broadening of the distribution even when the blinking on/off lifetime is two orders of magnitude shorter that the stroboscopic exposure time. The work is done by my colleague Simon Stein, and 
it tells that the impact is also related to the ratio $\frac{\tau_{o f f}}{\tau_{o n}}$. FCS is then applied to characterize these dynamics.

The effective on/off dynamic rate of fluorescent label Cy5 is determined from the fitting, as shown in Figure 30. Since the timescale of blinking kinetics is much shorter than the molecular diffusion, the dynamics are separable and the correlation model can be written in the same form as Eq. 19, where the fast timescale is related to the blinking dynamics as 120 :

$$
g_{p}(t)=\frac{\tau_{o n}}{\tau_{o n}+\tau_{o f f}}+\frac{\tau_{o f f}}{\tau_{o n}+\tau_{o f f}} \cdot e^{-\frac{\tau_{o n}+\tau_{o f f}}{\tau_{o n} \tau_{o f f}} \cdot t}
$$

The transition rates are listed in the following table from measurements under a broad range of excitation power (from ca. $5 \mathrm{~kW} / \mathrm{cm}^{2}$ to ca. $50 \mathrm{~kW} / \mathrm{cm}^{2}$ ).

\begin{tabular}{ccc}
\hline Excitation & $\tau_{\text {on }}$ & $\tau_{\text {off }}$ \\
\hline High & $1.1 \mu \mathrm{s}$ & $0.8 \mu \mathrm{s}$ \\
\hline Medium & $1.3 \mu \mathrm{s}$ & $1.1 \mu \mathrm{s}$ \\
\hline Low & $2.6 \mu \mathrm{s}$ & $2.2 \mu \mathrm{s}$ \\
\hline
\end{tabular}

These excitation powers cover the applied power density of $\mathrm{iPCH}$ experiments. In all the situations, both on and off lifetimes are changing, and they are comparable with each other. The transition process is also accelerated by increasing the excitation power. The same measurements have also been performed on another red emitting dye Alexa 647, which exhibits similar transition dynamics.

Molecules in nanochannels are under a different environment in contrast to the free solution. The frequent collision of molecule with the channel wall can potentially alter their fluorescence properties ${ }^{20}$. To check this influence, FCS measurement of Cy5 inside a Nanochannel is also performed, as shown in Figure 31. Here, molecular motion is described by 1D-flow as in Eq. 28 and in combination with the blinking dynamics, the model is written as:

$$
g(t)=\frac{1}{N} \cdot \frac{1}{\sqrt{1+4 D t / w_{x}^{2}}} \cdot e^{-\frac{t^{2}}{\left(\frac{w_{x}}{v_{x}}\right)^{2}\left(1+4 D t / w_{x}^{2}\right)}} \cdot\left(\frac{\tau_{o n}}{\tau_{o n}+\tau_{o f f}}+\frac{\tau_{o f f}}{\tau_{o n}+\tau_{o f f}} \cdot e^{-\frac{\tau_{o n}+\tau_{o f f}}{\tau_{o n} \tau_{o f f}} \cdot t}\right)
$$

The on/off lifetime is then extracted from the fitting. However, it should be mentioned that the FCS fit is actually sensitive to the start point of correlation lag time. For instance, if the model is applied to fit the correlation data points starting from $0.1 \mu \mathrm{s}$, the obtained 


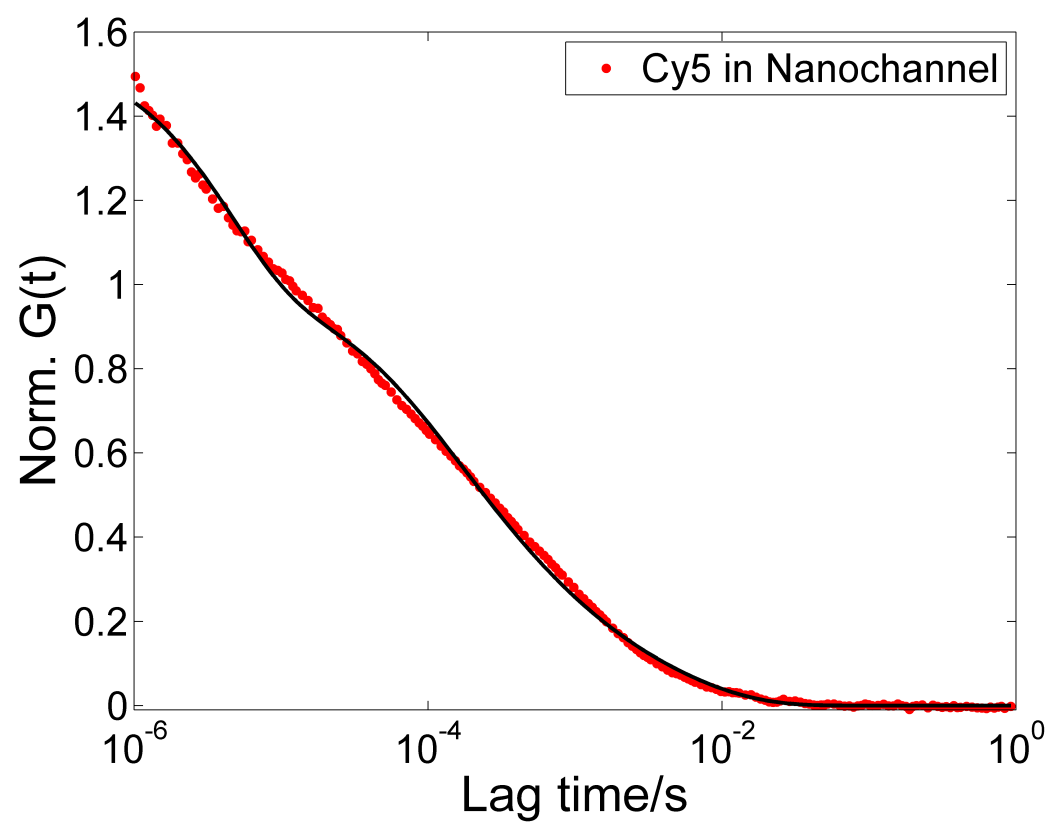

Figure 31: Characterization of blinking dynamics in nanochannel. FCS measurements of Cy5 in nanochannel is performed to extract blinking dynamics based on the model in Eq. 38 .

on/off lifetime can be varied. This is due to the presence of additional fast dynamics probably induced by the molecular interaction in nanochannels. The problem is associated to the inherent difficulty in fitting a mutli-exponential decay, where the ability to determine the precise values of each decay time and amplitude is greatly hindered by parameter correlation 67 . In principle, blinking on very fast timescale is averaged out and will not affect our brightness analysis. For this reason, the simple two state is assumed and the fitting is conducted by using reasonable start time from $0.1 \mu$ s to $1 \mu \mathrm{s}$. The achieved transition lifetimes have values in the range from $2.3 \mu \mathrm{s} \sim 12.0 \mu \mathrm{s}$ for $\tau_{\text {on }}$ and $2.0 \mu \mathrm{s} \sim 6.7 \mu \mathrm{s}$ for $\tau_{\text {off }}$. Nevertheless, since the excitation condition varies in the iPCH measurements, the characterized values of transition rates here are only for reference.

As a result, this blinking induced broadening of brightness distribution is modeled theoretically (credit: Simon Stein), with a variance adding into the previous background and EMCCD noise. Therefore, the overall variance is:

$$
\operatorname{var}_{\text {sum }}\left(\lambda, \tau_{\text {on }}, \tau_{\text {off }}, T_{\text {exp }}\right)=\operatorname{var}_{\text {det }}(\lambda, b)+\operatorname{var}_{\text {blink }}\left(\lambda, \tau_{\text {on }}, \tau_{\text {off }}, T_{\text {exp }}\right)
$$

Here $T_{\text {exp }}$ is the stroboscopic illumination time. In practice, a final brightness distribution can be well approximated by a Gaussian function with a mean $\lambda$ and the standard deviation 
$\sigma=\sqrt{\operatorname{var}_{\text {sum }}}$

\subsubsection{Single fluorophore brightness distribution}

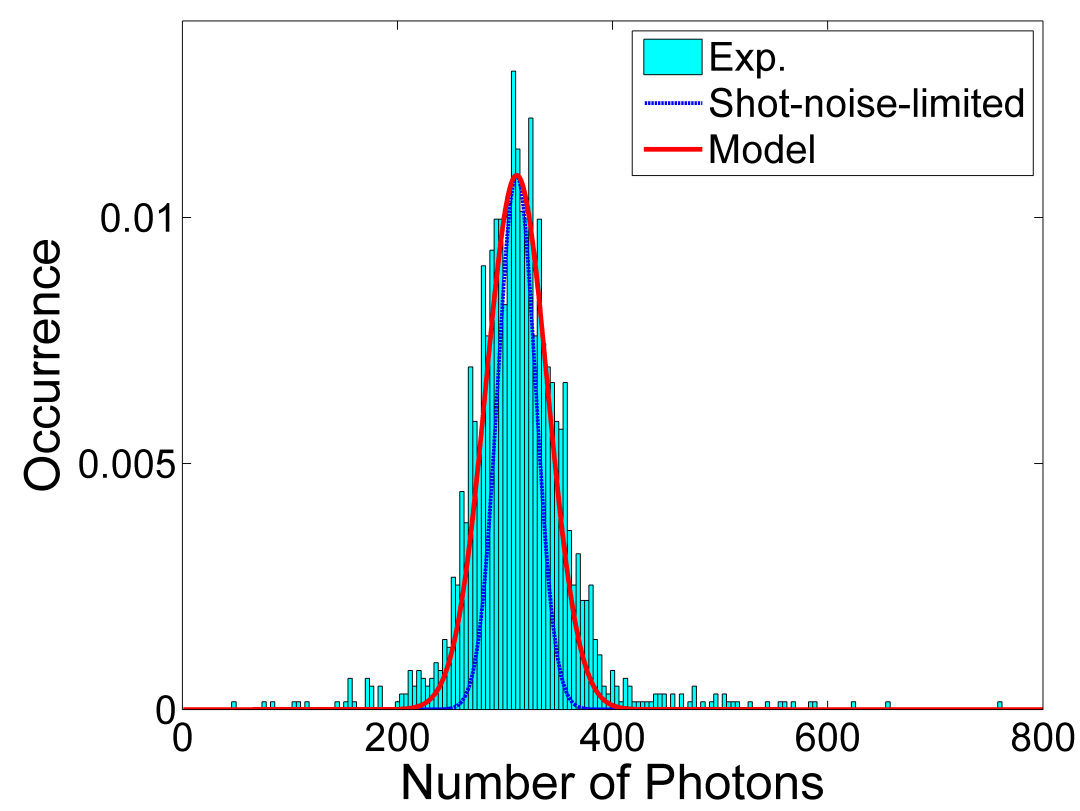

Figure 32: Brightness distribution of single fluorophores. The measured brightness distribution histogram of $\mathrm{Cy} 5$ is in good agreement with the theoretical model (red solid line).

A measurement of single Cy5 molecules in 1D Nanochannels is conducted to characterize the molecular brightness distribution. Previous work using burst size analysis requires careful characterization of multiple parameters such as light collection efficiency, laser excitation, molecular motion and photophysics to match a theoretical model 37 . Commonly, as uniform intensity bursts are desired, a fast flow rate is applied to compete against the random motion of molecular diffusion. It in the meantime reduces the photon budget due to the short residence time in detection volume. In contrast, iPCH measures the brightness distribution by just counting the photons from images of individual molecules.

In Figure 32 , it shows the brightness distribution of 1578 Cy5 molecules with an illumination time of $1 \mathrm{~ms}$. To make sure single-molecule emission patterns in this period can be well covered, an integration ROI pixel window of $12 \times 12(1.46 \mu m \times 1.46 \mu m$ in real space $)$ is used with background subtracted. Molecules in a relative uniform illumination region with a diameter of $4 \mu \mathrm{m}$ are taken into account, producing a normalized photon counting 
histogram. A model of simple Gaussian function with the variance based on Eq. 36 is also demonstrated, which exhibits reasonable agreement with the measured distribution. The slightly broader histogram of the experimental results is due to the impact of photoblinking. As a comparison, corresponding shot-noise-limited distribution is also plotted. Furthermore, two other fluorescent dyes are checked in the same channel. Figure 33 shows the good agreement and consistent performance of iPCH method.
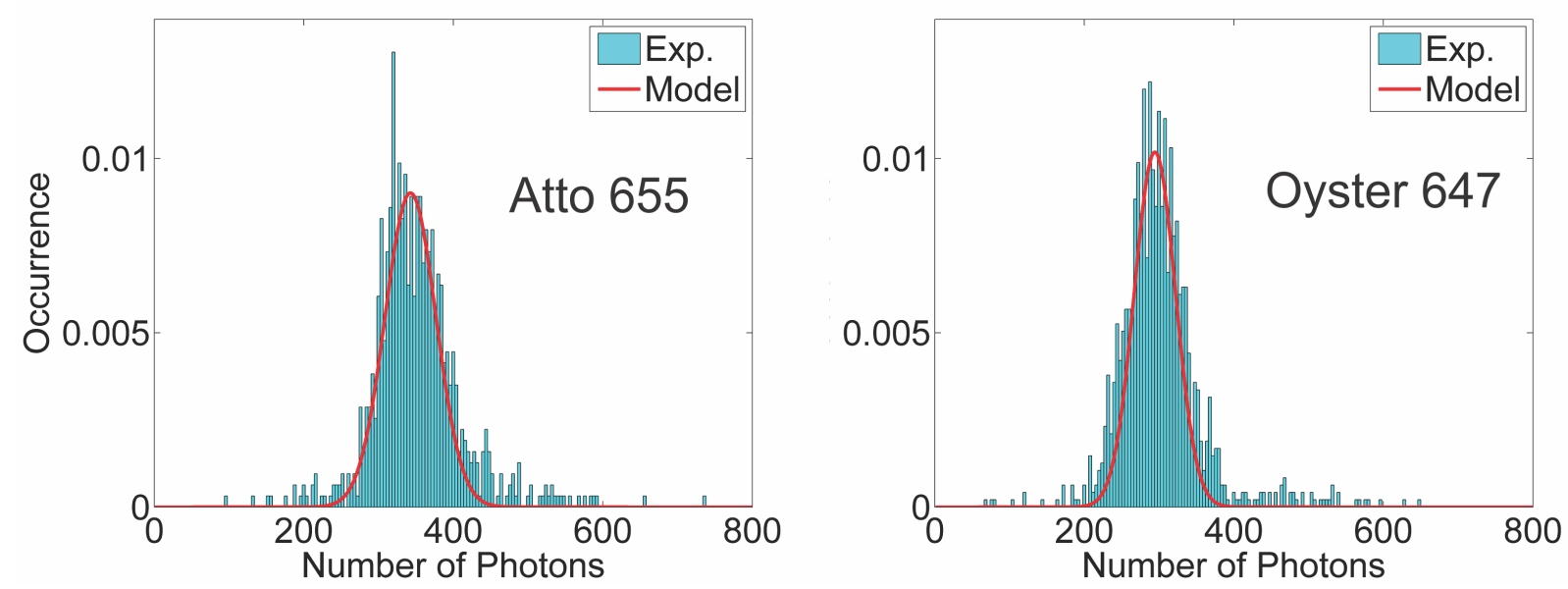

Figure 33: Brightness distribution of other two fluorophores. In both cases, the measured photon counting histogram is in good agreement with the Gaussian model.

\subsection{Applying iPCH to reveal the distribution of molecular mix- tures}

\subsubsection{Disentangling the mixtures of two dyes}

The detected photons carry the information of emission spectra, polarization or arrival time, which is usually exploited to discriminate different molecules. With the accuracy of the iPCH method, the brightness information can be used for the identification of single molecules. Here, two fluorophores with substantial emission spectral overlap are measured to demonstrate the resolving power of the $\mathrm{iPCH}$ method.

Under the same stroboscopic illumination time of $1 \mathrm{~ms}$, two red-emitting fluorophores Cy5 and Oyster 647 are respectively transported and measured inside the 1D Nanochannels. With 
the sample concentration of $5 \mathrm{nM}$ and constant excitation condition, individual molecules are well-isolated and then analyzed by the iPCH routine. Figure 34 (A) and (B) show the brightness histograms for both samples out of 1499 and 1245 molecules, respectively. They both show single peak distribution and can be well fitted by a single Gaussian model.
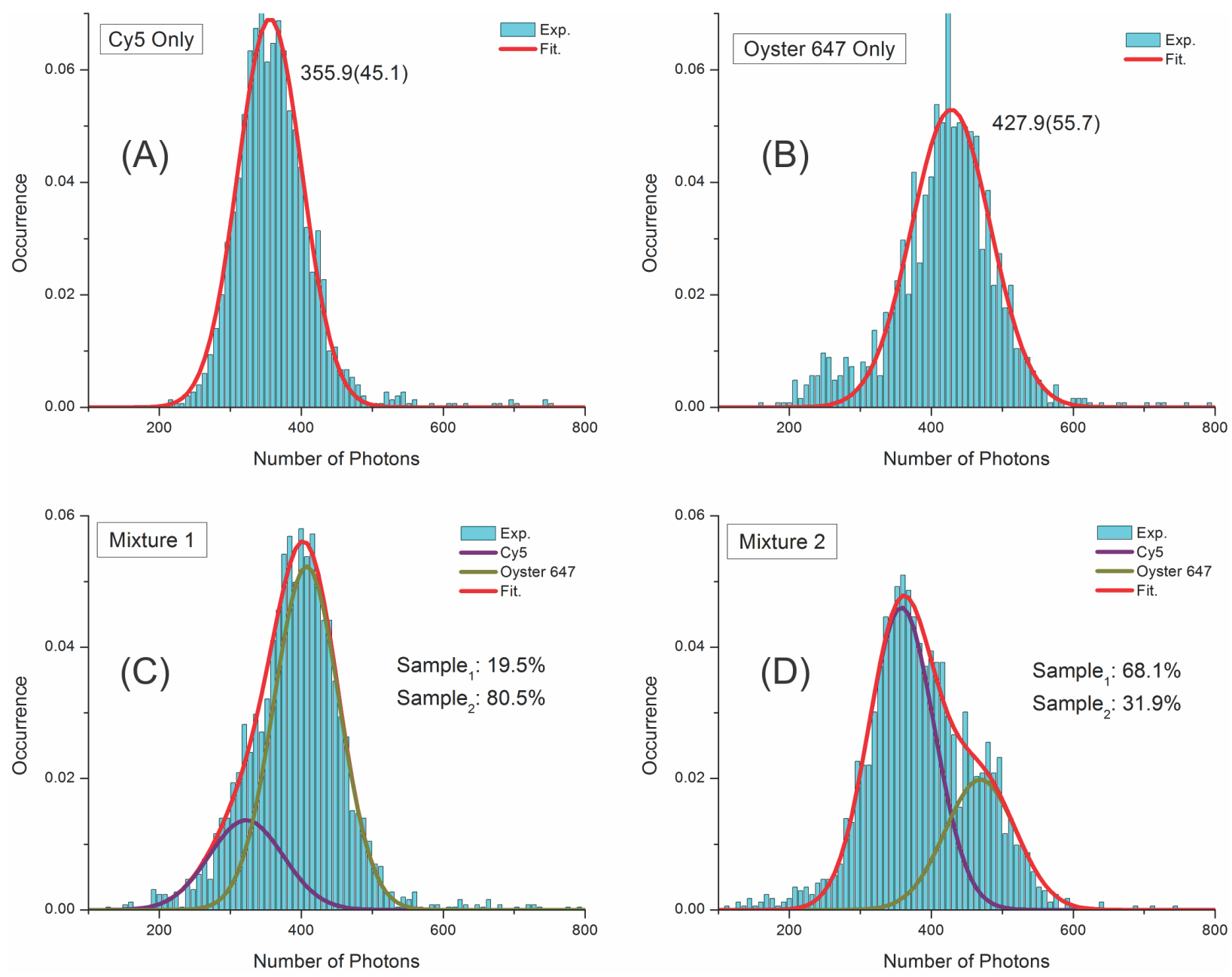

Figure 34: Identification of single molecules. iPCH analysis of fluorescent brightness distribution for two fluorophores and their mixtures. (A) Sample solution with only Cy5. (B) Sample solution with only Oyster 647. (C) Sample solution with Cy5 and Oyster 647 with a mixing ratio of 1:3. (D) Sample solution with Cy5 and Oyster 647 with a mixing ratio of 3:1.

Subsequently, the mixtures of those two fluorophores are measured under the same experimental condition. It requires a meticulous cleaning procedure in order to get rid of the sticky molecules. By applying a high electrokinetic force and adding buffer solution, channels can be quickly washed and then refilled with another sample solution. Two other samples of 
Cy5 and Oyster 647 mixture are also measured in the same condition as before, the mixing ratios are set as 1:3 and 3:1 (Cy5: Oyster 647). As shown in Figure 34(C) and (D), broader histograms have been obtained, showing a very noticeable contrast in shape and the single Gaussian model is no longer feasible for a reasonable fitting. A two-component Gaussian mixture model allows a good fit for the data of mixtures and the information of brightness differences from previous measurements is used to put a constraint on the fitting. Though the difference of mean brightness for the two fluorophores is only around $\sim 20 \%$ from each other, the mixing ratios still can be reasonably revealed. Simply by integrating the area of each Gaussian component, compositions of each sample are estimated as (19.5 \pm 2.9$) \%$ Cy5: $(80.5 \pm 2.9) \%$ Oyster647 and $(68.1 \pm 3.0) \%$ Cy5: (31.9 \pm 3.0$) \%$ Oyster647. Here, datasets of mixtures are obtained from 2585 and 1726 localized molecules. A bootstrap method has been applied to estimate the fitting errors, where brightness values from each dataset have been resampled 30 times. Fitting results of brightness histogram from those 30 datasets allow to estimate the mean ratio of each species and its error. It is noted that the ratio of Cy5 is underestimated in both cases, which could result from the uncertainty of concentration determination or the potential different adsorption properties of those two dyes to the channel surface.

As the emission property is governed by Poisson statistics, the brightness histogram can be better separated by recording more photons from each individual molecule. When a desired resolving capability is achieved, this brightness characterization method can be developed into a real-time single-molecule sorting system by combining the method with electrokinetic manipulation, which has already attracted much interest in single-molecule epigenetic analysis $\stackrel{18}{18}$ and would open tremendous opportunities for further biomedical studies. Meanwhile, the platform is inherently designed to be compatible with the multicolor application, where the stroboscopic imaging can be facilitated by a pulsed interleaved excitation ${ }^{85}$ mode to realize the multiplexed single-molecule identification.

\subsubsection{DNA sizing}

It has been well-known that double-stranded DNA (dsDNA) can be stoichiometrically stained with intercalating dyes. Thus, the number of labeled dyes on the base pairs is proportional 
to the DNA length, which allows using fluorescence brightness for DNA size. Measuring the size of nucleic acid fragments has been performed for many years, with different methods $\frac{43}{4}$. Long DNA strands can be stretched on the surface or inside nanochannels to map its size or barcode $\underline{93}$. For short or randomly coiled DNA, the measured diffusion coefficient can represent its size. However, resolving a DNA mixture with different length is challenging. Fluorescence burst analysis has been applied in such case, where BSD is successfully investigated to reveal the length distribution ${ }^{45}$. Since the iPCH is a simple but powerful tool to determine the brightness distribution of different species, the method is employed to measure the mixture of small $0.5 \mathrm{kbp}$ and $1 \mathrm{kbp}$ DNA fragments labeled with intercalating YOYO-1 dyes. As the staining ratio also limits the size resolution ${ }^{43}$, fragments that are smaller than $0.5 \mathrm{kbp}$ is less suited for this type of measurements.

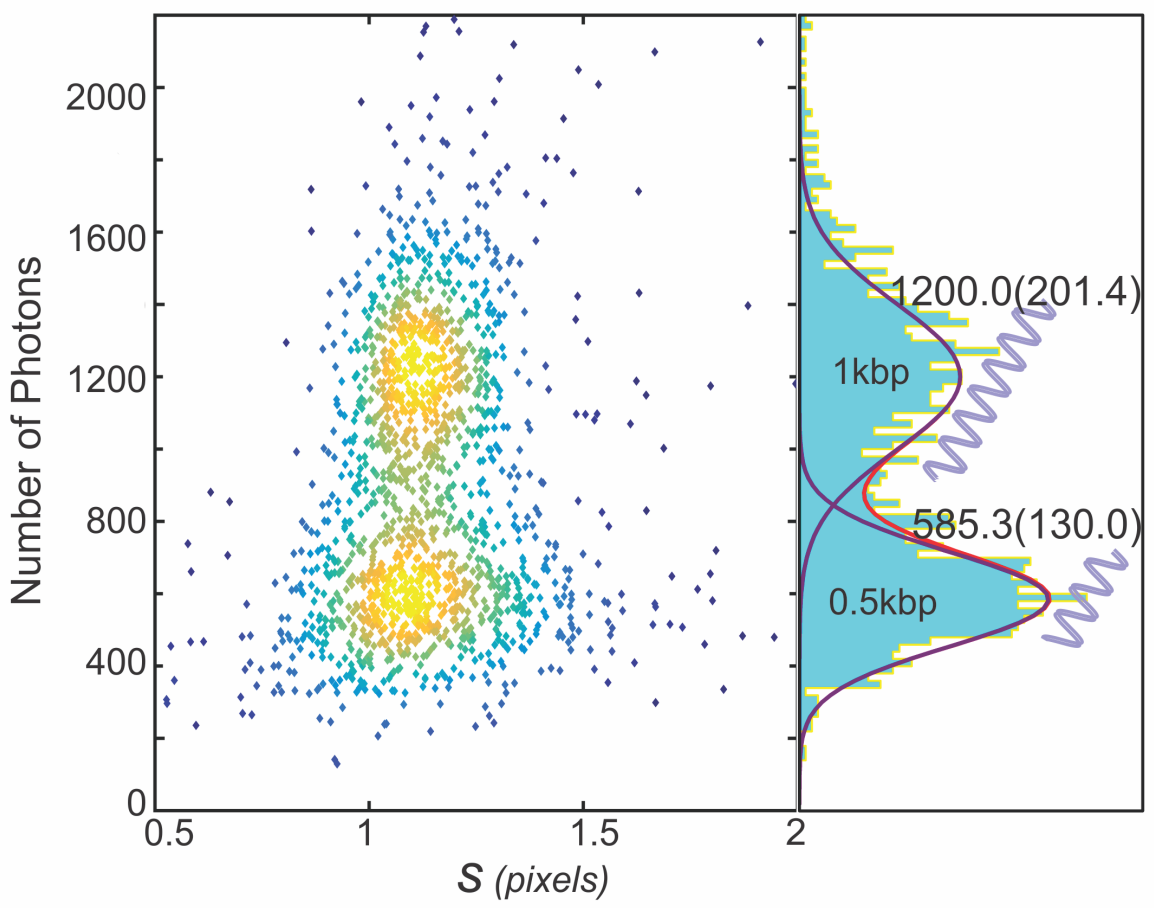

Figure 35: DNA sizing. The brigthness of individual molecules is plotted against their localization uncertainty. $\boldsymbol{S}$ represents the fitting standard deviation.

A 2D nanochannel is used in combination with a stroboscopic illumination time of $2 \mathrm{~ms}$. As shown in Figure 35, the iPCH analysis is demonstrated along with the standard deviations $S$ from 2D symmetrical Gaussian fitting for single molecules. It is noted that at lower photon 
counts, a broader distribution of $\boldsymbol{S}$ is obtained. Two species can be distinguished based on their mean brightness, and their difference is 2.05 fold, which is in great agreement with the DNA lengths.

\subsubsection{Determining distributions of stoichiometries for molecular mixtures}

The more interesting application is to probe the stoichiometry labeled subunits in a molecular complex, which requires a very high sensitivity so that the brightness discrimination is possible. For this purpose, samples of DNA fragments (42bp) with heterogeneous labeling numbers are measured, which mimic the situation of protein oligomerization. There, three covalent bonds are chemically modified on the DNA structure and selectively labeled by fluorescent dye Atto 647N. The sample quality has been characterized by using fluorescence antibunching, as shown in Figure 8 (b) of Chapter 2, where it can only extract a value of the average emitters number. To determine the full distribution of each species, the iPCH method is employed in a 2D nanochannel using a excitation pulse length of $2 \mathrm{~ms}$.

Figure 36 shows both the scattering plot and the brightness histogram, where three species can be distinguished, and their mean photon counts match well with the expected labeling stoichiometry. Sample distribution of three species is revealed from the iPCH analysis by using three-component Gaussian fitting, as shown in Figure 37. The information can be compared with estimated average number of emitters from the photon antibunching method, as discussed in Chapter 2. In Figure 8 b, the same sample has been measured in free solution by photon antibunching method and given an average emitter number of 2.37 per complex. Using relation in Eq. 23 (in Chapter 2), average number of emitters calculated from the species distribution measured by $\mathrm{iPCH}$ is 2.19 , which reasonably matches with the antibunching estimation. In contrast, the measurements of maximum 2 and 4 Atto647n labeled DNA gives distinct values of 1.75 and 2.60, as shown in Figure $8 \mathrm{c}$ and d.

For Poissonian statistics, the standard deviation is proportional to the square root of the mean value. Therefore, the brightness difference between $\mathrm{N}$ and $\mathrm{N}+1$ labeled species becomes less discernible when $\mathrm{N}$ is getting larger. Collecting more photons would certainly improve 


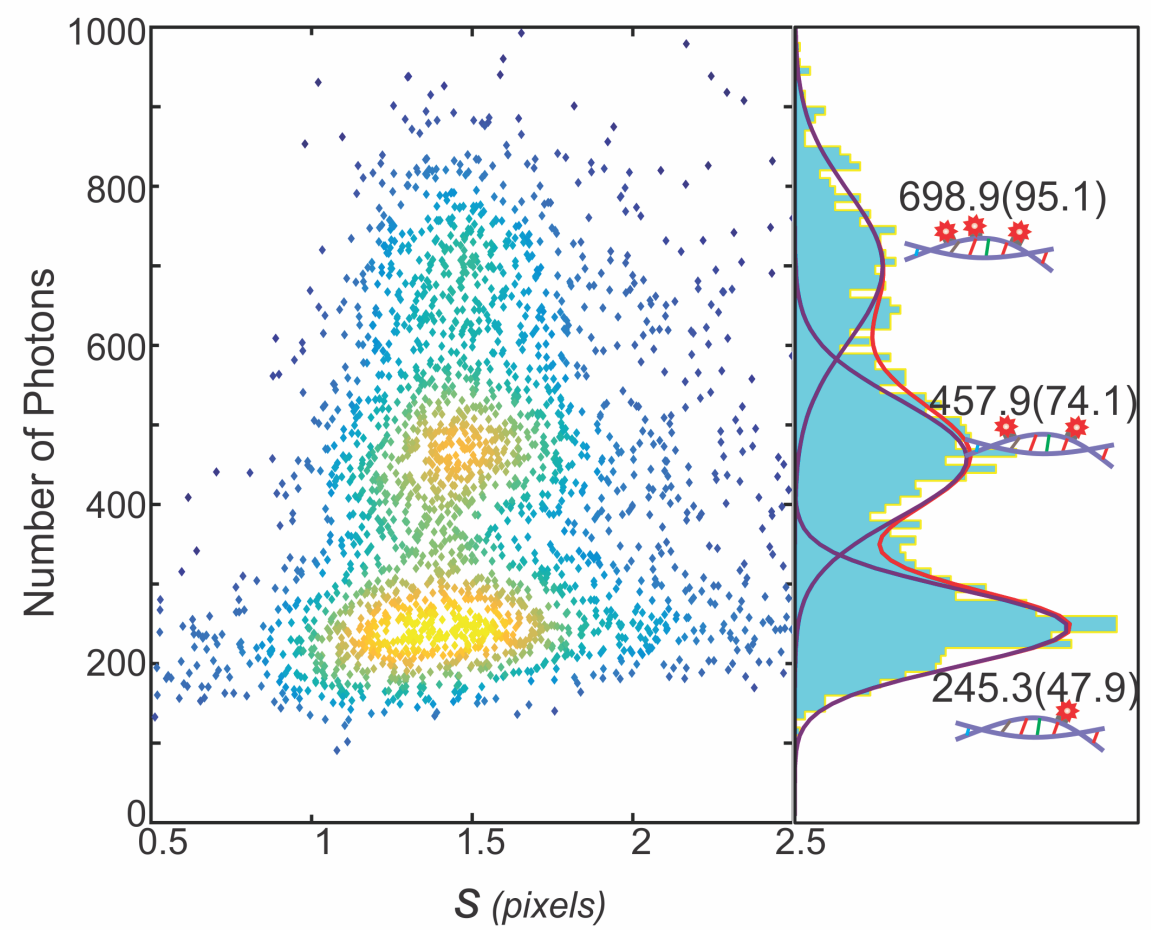

Figure 36: Single-molecule stoichiometry. DNA with one, two and three Atto 647N labeled sample species can be well revealed from the iPCH analysis.

the resolving ability. Nevertheless, in the case of small photon budget, given that the brightness distribution of each species can be precisely characterized and follows well with the stoichiometric ratio, a deconvolution method might also improve the resolving power of the iPCH method ${ }^{7}$. As this imaging-based chip-like platform enables the parallel investigation of multiple molecules at one time, all the experiments can be performed within a few minutes and with minimum sample volume.

Only very few fools are capable of measuring the brightness distribution of single molecules in solution. Particularly, no other method has realized high-speed stoichiometric sensitivity down to the single-molecule level. Thus, the iPCH method can help to fill the technological gap for quantitative single-molecule investigation. The experiment illustrated here is similar to protein aggregation studies, where the proportion of different oligomeric species such as monomers, dimers, and trimers needs to be quantified within a mixed solution. The iPCH method is able to determine this distribution with few experimental constraints and high accuracy. It is ready to be implemented into a conventional microscopy system and holds potential to solve real biological problems. 


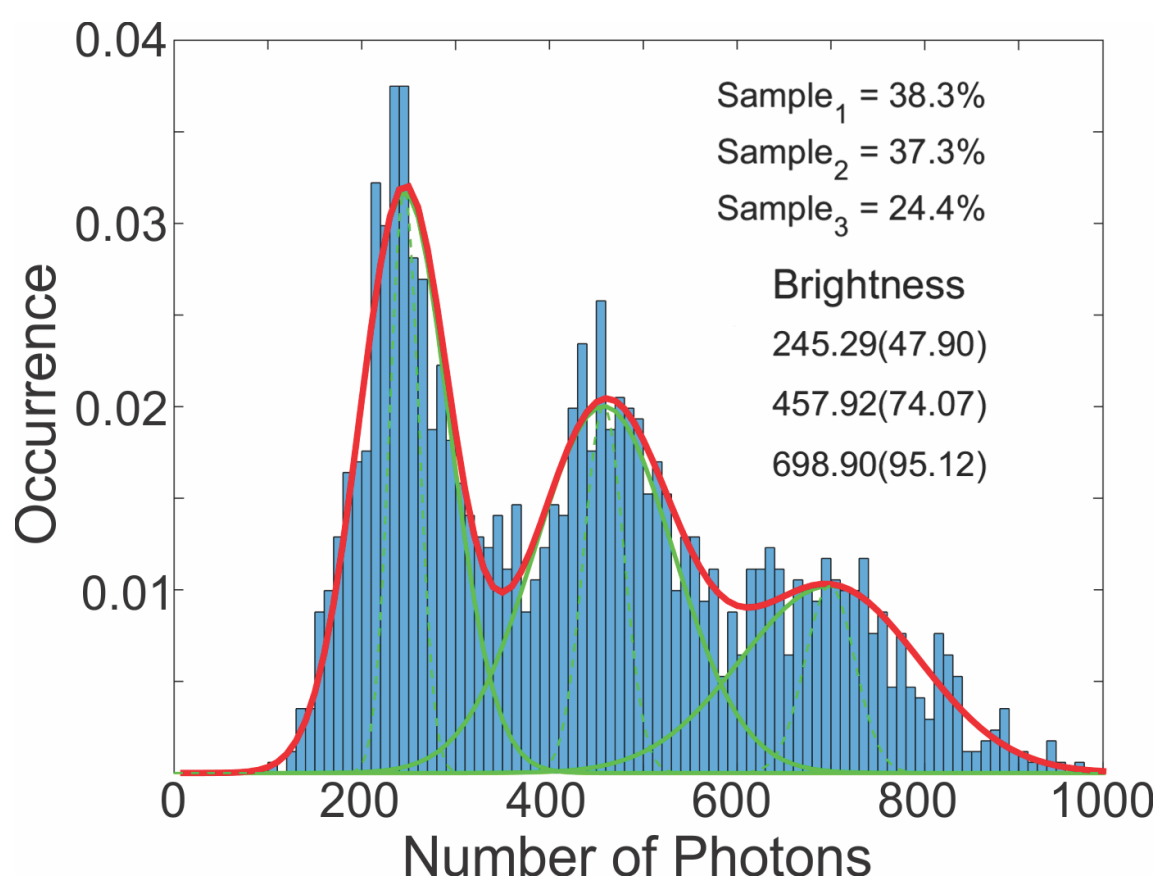

Figure 37: iPCH analysis to reveal the sample distribution. The population of one, two and three Atto $647 \mathrm{~N}$ labeled sample species is extracted from a three-component Gaussian fitting of the brightness histogram.

\subsection{Estimating diffusion coefficient by spot size analysis}

In solution, the translational diffusion coefficient is connected with molecular hydrodynamic radius via the Stokes-Einstein relation ${ }^{34}$. By measuring the diffusion coefficient of a fluorescently labeled molecule, changes of molecular size can be monitored and it has found numerous applications in biology and chemistry over the recent decades 122 . Among all the well-established methods of determining the diffusion coefficient, FCS and Single-particle tracking (SPT) are the most widely applied techniques at nanomolar sample concentrations. FCS is typically measured in a confocal-based detection scheme and SPT is an imaging-based method that can directly visualize the molecular motion in real-time.

SPT determines the diffusion coefficient from the video of moving particles. There, location of each particle is obtained from every image frame, thus in consecutive recording single particle locations from multiple frames enables to produce a trajectory of each particle. Analyzing lots of these time-correlated trajectories can help quantify the diffusion coefficient. In practice, this method is based on the mean-square displacement (MSD) analysis ${ }^{92}$, which typically requires more than 20 consecutive locations for each single trajectory to ensure an 
accurate estimation ${ }^{[25]}$. This condition is extremely difficult to be achieved when measuring single fluorophores or small biomolecules of only several nanometers in size. Due to the fast Brownian motion, these molecules only reside at the focal plane for a short moment (a few milliseconds or less), in which period a EMCCD camera can neither capture multiple image frames nor precisely localize the molecule because of the limited photon budget. Nonetheless, a previous work estimated diffusion coefficient based on the spots size obtained from multiple single-molecule snapshots $[102$ and it thus, found a favorable applicability in fast moving small molecules and is suitable for stroboscopic single-molecule imaging in nanofluidic device.

As the channels dimension along the optical axis is smaller than the focus size, molecules are always kept in focus. Single-molecule imaging patterns are described as the convolution of the detection PSF with the occupation frequency of certain positions during the illumination time. The latter part is governed by diffusion and the observation time window. Fast moving molecules or long illumination times will lead to smeared or blurred single-molecule image patterns. In the stroboscopic image mode, it is convenient to set up various illumination times and detected single-molecule patterns can provide a good estimation of the diffusion coefficient.
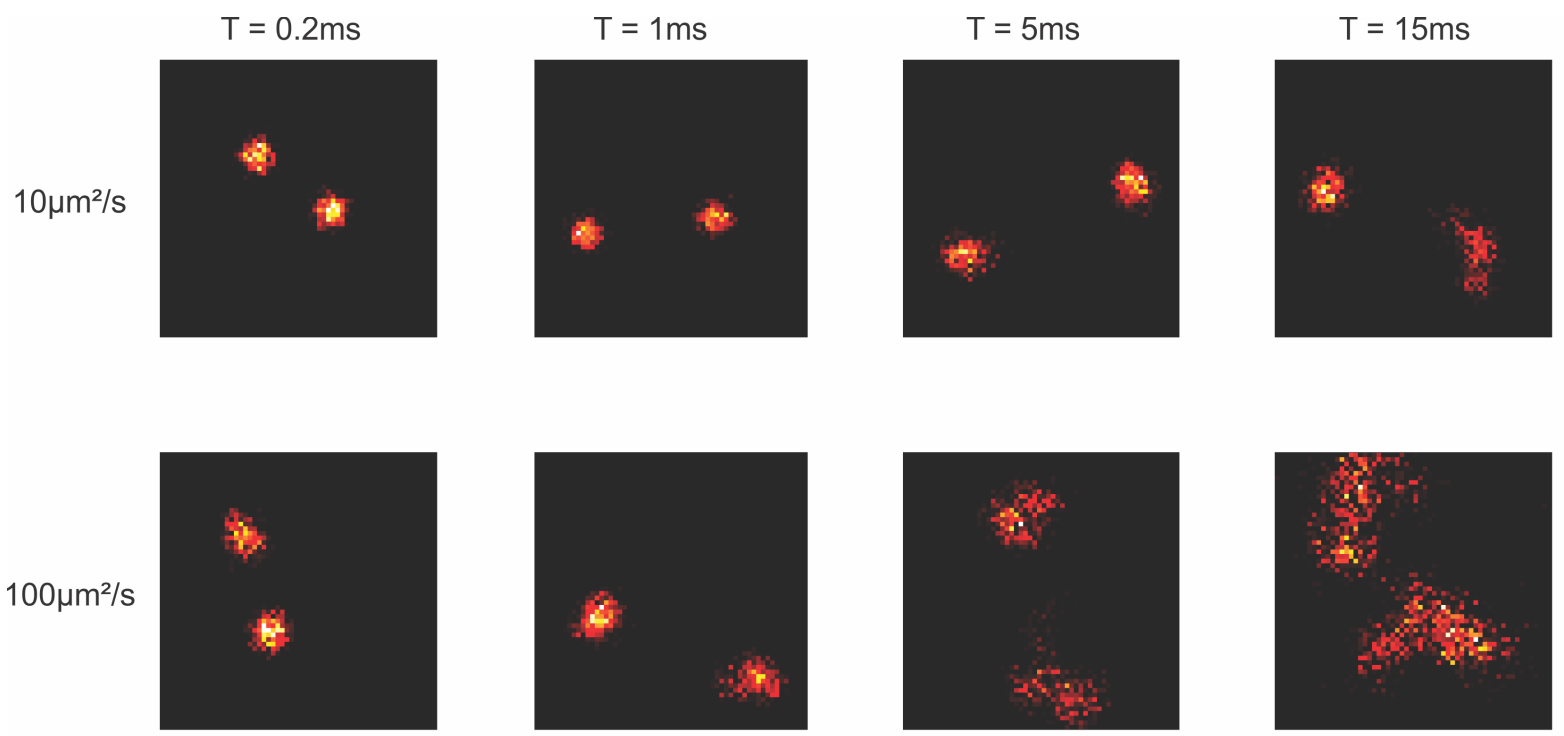

Figure 38: Simulated single-molecule imaging patterns with different illumination time. The situation of two diffusion coefficients is also compared. 
Here, the underlying theoretical model is briefly recalled, and simulated images are used to test this estimation method.

As shown in Figure 38, under different illumination pulses, various imaging patterns of single molecules were generated from a multi-step 2D random walks simulation. It is performed without too much difference from that described before (Section 4.1), expect two diffusion coefficients are used under different stroboscopic illumination pulses. The simulation time step is $10 \mu s$ and within this time the simulated image is represented by a Gaussian-shaped detection PSF with a standard deviation of $150 \mathrm{~nm}$. The corresponding single-molecule image is an accumulative image under a certain illumination time and is displayed in one pixelated image frame with pixel size of $70 \mathrm{~nm}$. The fast diffusion case shows a dramatical change of patterns that exhibits as irregular shapes under long illumination time.

In the analytical calculation, the projection of an in-focus immobilized fluorescence molecule at position $\mathbf{r}$ onto a $2 \mathrm{D}$ image is given as detection PSF, which can be well approximated by a Gaussian function that reads:

$$
f_{0}(\mathbf{r})=I_{0} \cdot e^{-\frac{\left(\mathbf{r}-\mathbf{r}_{0}\right)^{2}}{2 \sigma_{0}^{2}}}
$$

Here $I_{0}$ is a multiplication factor that represents the amplitude of fitting function. The final imaging spot of the single-molecule is then given by the convolution of this PSF with a molecular diffusion trajectory $p(\mathbf{r} \mid T)$ during the illumination time $T$. So, there is the observed single-molecule PSF:

$$
f_{d}(\mathbf{r} \mid T)=p(\mathbf{r} \mid T) * f_{0}(\mathbf{r})
$$

Here $p(\mathbf{r} \mid T)$ is a conditional probability density that describes the occupation frequency of certain positions during the exposure time $T$ and has the form:

$$
p(\mathbf{r} \mid T)=\int_{0}^{T} \frac{1}{8 \pi D t} e^{-\frac{\left(\mathbf{r}-\mathbf{r}_{0}\right)^{2}}{4 D t}} d t
$$

where $\mathbf{r}_{0}$ is the original molecular position and $D$ is the diffusion coefficient.

The single-molecule pattern is broadened by diffusion and thus has a bigger variance, where the relation

$$
\sigma^{2}=\sigma_{0}^{2}+\sigma_{d}^{2}
$$

holds. Here $\sigma^{2}$ can be directly achieved from the fitting of observed single-molecule patterns with 2D Gaussian function. $\sigma_{d}^{2}$ is related to both diffusion coefficient and illumination time. 
Therefore, it gives

$$
\sigma^{2}=\sigma_{0}^{2}+c D T
$$

Here, $c$ is a constant that is related to the dimension of diffusion model. In 2D Nanochannels, the $2 \mathrm{D}$ diffusion model is well satisfied and $c=1 / 3^{102}$.

In practice, several different illumination times are used and multiple image stacks can be used to extract the different values of $\sigma^{2}$. The simple linear relation of Eq. 44 enables the diffusion coefficient to be extracted. It should be noted that the $\sigma_{d}^{2}$ here has a physical meaning of variance with respect to average position of the trajectory, instead of the start position of the trajectory that is used in the SPT method. This is due to the fact that this analysis of spot size determines the diffusion coefficient without knowing the sequence of imaging recording ${ }^{29}$. Random walk simulations with given diffusion coefficients are also used to validate this method.

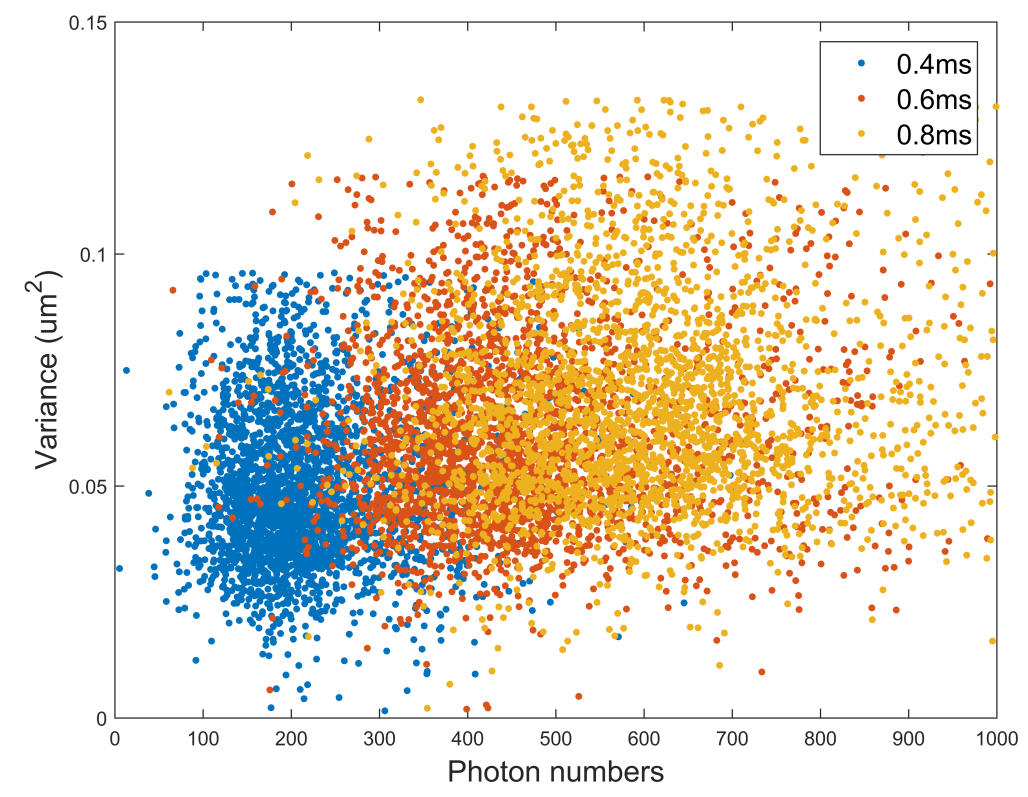

Figure 39: $\mathrm{iPCH}$ analysis of simulated images under three different excitation pulse lengths. The variance is calculated from $2 D$ Gaussian fitting of each molecule.

Using the simple linear relation of Eq. 44, different values of $T$ can be opted to reveal the diffusion coefficient $D$. Therefore, the advantage of stroboscopic imaging is naturally implemented, and the additional information of localization uncertainty that has been presented 
before can now be applied to evaluate single-molecule spots.

Figure 39 shows an overlapped scattering plot of iPCH analysis from simulated images using three different illumination times. A fast diffusion coefficient of $100 \mu \mathrm{m}^{2} / \mathrm{s}$ is set for simulation and the EMCCD multiplicative noise is also included. A constant count rate of $5 \times 10^{5}$ counts per second per molecule and background $5 \times 10^{3}$ counts per second per pixel are set to approximate a real experimental condition.

Furthermore, in Figure 40, the mean values of variance under four different illumination times are shown. The simulated diffusion coefficients of $10 \mu \mathrm{m}^{2} / \mathrm{s}$ and $100 \mu \mathrm{m}^{2} / \mathrm{s}$ are used respectively. In both cases, a simple linear fitting is applied and the value $D$ is inferred from the slope of each curve that has the value $10.4 \mu \mathrm{m}^{2} / \mathrm{s}$ and $104.3 \mu \mathrm{m}^{2} / \mathrm{s}$. It is worth to mention that, this determination method has a desirable performance when the Gaussian model can well approximate the molecular patterns. Thus, stroboscopic imaging of too long laser pulses is not preferred, even though more photons can be recorded. Given that a reasonable count rate of $5 \times 10^{5}$ counts per second per molecule could be reached, simulation results show that using pulse lengths from $0.2 \mathrm{~ms}$ to $1 \mathrm{~ms}$ can already reveal both the slow and fast diffusion coefficients accurately.

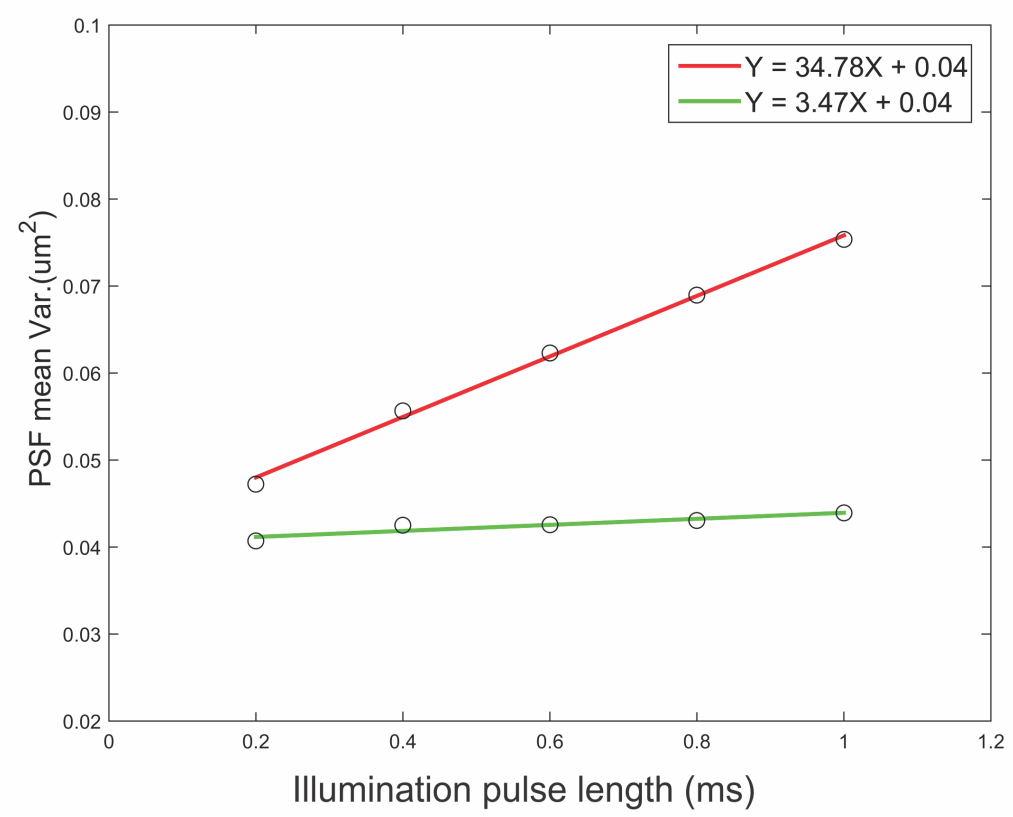

Figure 40: Calculation of diffusion coefficient from the spot-size analysis. Diffusion coefficients are obtained from the slope of each curve according to Eq. 44. 


\section{Conclusions and outlook}

To summarize, the work presented in this dissertation investigated two fluorescence methods for probing molecular stoichiometry in solution. First, photon antibunching was used to estimate the average emitter numbers on a molecular complex and was subsequently applied as a tool to study labeling efficiency. Nanofluidic devices were then introduced to facilitate the detection of single molecules in solution and accurately measure their brightness. A new method of iPCH was developed to determine the in vitro single-molecule stoichiometry with high throughput.

To the first time, direct imaging of fast moving single fluorophores was demonstrated in nanofluidic channels. With this simple but novel combination, nanofluidics assisted singlemolecule fluorescence imaging has been demonstrated as a versatile platform for measuring single-molecule properties. The equipment is ready to be implemented into a conventional bio-imaging system, which holds potential for widespread biophysical and biomedical applications. Furthermore, when electrokinetic flow is applied to manipulate single-molecule motion, the imaging system can monitor this process in real-time. This allows performing single-molecule trapping experiments without using sophisticated feedback control. The iPCH method, with its simplicity and high accuracy, has been validated by experiments on both single fluorophores and molecular complexes with multiple labels. It can be used for various biological problems, such as disentangling oligomeric species during protein fibril formation or revealing the protein aggregation pathway at its early stage 104 .

Photon antibunching and iPCH measure the stoichiometry from different perspectives. Photon antibunching uses a confocal spectroscopy together with pulsed excitation and fast single photon detectors, which enables the simultaneous determination of other fluorescence properties such as lifetime. The experimental configuration is also compatible to work on cultured living cells and holds the potential to carry out in vivo molecular counting. iPCH takes advantage of the feature of the nanofluidic device which is able to spatially separate molecules in solution. This has advantages for characterizing the distribution of molecular properties in vitro. Thus, iPCH is more useful for molecular diagnostics where minimum sample consumption and high precision are required.

In the future, the ability of precise brightness determination could be utilized in more com- 
plex setups, such as expanding the iPCH system into a multi-color excitation/detection modality. It allows measuring smFRET in nanofluidic devices to study protein conformational dynamics and intramolecular interactions without the surface tethering 115 . Moreover, the multi-color system is very practical in multiplexed single-molecule detection, where single molecules of different colors can be detected and quantified in single measurement. It is also of great interest to employ the photoactivatable fluorophores that can work on the biological problems with extended concentration range ${ }^{77}$.

Aside from the brightness analysis this dissertation concentrates on, it is certainly possible to measure other molecular parameters. Since images obtained by using nanoconfinement are similar to the results from immobilized single molecules, the imaging system can be combined with other well-established single-molecule techniques. Investigation of light properties such as anisotropy ${ }^{[6]}$, spectra ${ }^{126}$ or scattering ${ }^{39}$ would enable a multi-parameters analysis in solution. Furthermore, the system will benefit from recent and future advances in micro/nanofabrication. Notably, techniques have been recently developed to reduce the cost of 1D nanochannels drastically [17, and incorporate nanostructures inside the channels, which produces a potentially even more versatile platform 31 . For instance, plasmonic nanoantenna can be integrated into nanochannels to sense biochemical changes or enhance fluorescence 31 . Also, nanofluidic devices with multiple metal electrodes enable the concurrent manipulation of multiple molecules in the channels.

Although technological advancements allow increasingly better investigations of biological processes, no tool comes entirely without drawbacks. Inevitably, a molecule in the nanoconfined environment is more perturbed than in free solution. It may also adhere to the channel walls or undergo specific chemical interactions ${ }^{20}$. Caution should be taken when modifying the surface properties, which could also impact the electrokinetic flow. As charges and ion clouds are often part of or surrounding a real biomolecule, the external electrical field can create an additional force that may alter the molecular properties. Additionally, experiments of probing single-molecule stoichiometry require photon emission from all labels to be indistinguishable and independent. Energy transfer occurs when fluorescence labels are close together, it may strongly influence the result of the analysis. Therefore, rather than relying on a single experimental method, a thorough single-molecule study should combine information from multiple different methods. I hope that photon antibunching and iPCH 
will become useful methods that routinely applied to solve various problems in the future. 


\section{References}

[1] Patrick Abgrall and Nam Trung Nguyen. Nanofluidic devices and their applications. Analytical chemistry, 80(7):2326-2341, 2008.

[2] Patrick Abgrall and Nam-Trung Nguyen. Nanofluidics. Artech House, 2009.

[3] W Patrick Ambrose, Peter M Goodwin, Jörg Enderlein, David J Semin, John C Martin, and Richard A Keller. Fluorescence photon antibunching from single molecules on a surface. Chemical physics letters, 269(3):365-370, 1997.

[4] Daniel Axelrod. Total internal reflection fluorescence microscopy. Methods in cell biology, 30:245-270, 1989.

[5] Niccolò Banterle and Edward A Lemke. Nanoscale devices for linkerless long-term single-molecule observation. Current opinion in biotechnology, 39:105-112, 2016.

[6] Th Basché, WE Moerner, M Orrit, and H Talon. Photon antibunching in the fluorescence of a single dye molecule trapped in a solid. Physical review letters, 69(10):1516, 1992.

[7] Cyrus W Beh, Deng Pan, Jason Lee, Xuan Jiang, Kelvin J Liu, Hai-Quan Mao, and Tza-Huei Wang. Direct interrogation of dna content distribution in nanoparticles by a novel microfluidics-based single-particle analysis. Nano letters, 14(8):4729-4735, 2014.

[8] Eric Betzig. Proposed method for molecular optical imaging. Optics letters, 20(3):237239, 1995.

[9] Eric Betzig, George H Patterson, Rachid Sougrat, O Wolf Lindwasser, Scott Olenych, Juan S Bonifacino, Michael W Davidson, Jennifer Lippincott-Schwartz, and Harald F Hess. Imaging intracellular fluorescent proteins at nanometer resolution. Science, 313(5793):1642-1645, 2006.

[10] Erez Boukobza, Alan Sonnenfeld, and Gilad Haran. Immobilization in surface-tethered lipid vesicles as a new tool for single biomolecule spectroscopy. The Journal of Physical Chemistry B, 105(48):12165-12170, 2001. 
[11] R Hanbury Brown and Richard Q Twiss. Correlation between photons in two coherent beams of light. Nature, 177(4497):27-29, 1956.

[12] Han Cao, Zhaoning Yu, Jian Wang, Jonas O Tegenfeldt, Robert H Austin, Erli Chen, Wei Wu, and Stephen Y Chou. Fabrication of $10 \mathrm{~nm}$ enclosed nanofluidic channels. Applied physics letters, 81(1):174-176, 2002.

[13] HJ Carmichael and DF Walls. A quantum-mechanical master equation treatment of the dynamical stark effect. Journal of Physics B: Atomic and Molecular Physics, 9(8):1199, 1976.

[14] Francis Cayer. Rectangular flat-top beam shaper, July 15 2008. US Patent 7,400,457.

[15] Yan Chen, Joachim D Müller, Peter TC So, and Enrico Gratton. The photon counting histogram in fluorescence fluctuation spectroscopy. Biophysical journal, 77(1):553-567, 1999.

[16] Yan Chen, Li-Na Wei, and Joachim D Müller. Probing protein oligomerization in living cells with fluorescence fluctuation spectroscopy. Proceedings of the National Academy of Sciences, 100(26):15492-15497, 2003.

[17] Stephen Y Chou, Chris Keimel, and Jian Gu. Ultrafast and direct imprint of nanostructures in silicon. Nature, 417(6891):835-837, 2002.

[18] Benjamin R Cipriany, Patrick J Murphy, James A Hagarman, Aline Cerf, David Latulippe, Stephen L Levy, Jaime J Benítez, Christine P Tan, Juraj Topolancik, Paul D Soloway, et al. Real-time analysis and selection of methylated dna by fluorescenceactivated single molecule sorting in a nanofluidic channel. Proceedings of the National Academy of Sciences, 109(22):8477-8482, 2012.

[19] Benjamin R Cipriany, Ruqian Zhao, Patrick J Murphy, Stephen L Levy, Christine P Tan, Harold G Craighead, and Paul D Soloway. Single molecule epigenetic analysis in a nanofluidic channel. Analytical chemistry, 82(6):2480-2487, 2010.

[20] Adam E Cohen. Trapping and manipulating single molecules in solution. PhD thesis, Stanford University, 2006. 
[21] Adam E Cohen and Alexander P Fields. The cat that caught the canary: what to do with single-molecule trapping. ACS nano, 5(7):5296-5299, 2011.

[22] Adam E Cohen and WE Moerner. Method for trapping and manipulating nanoscale objects in solution. Applied physics letters, 86(9):093109, 2005.

[23] Adam E Cohen and WE Moerner. Suppressing brownian motion of individual biomolecules in solution. Proceedings of the National Academy of Sciences of the United States of America, 103(12):4362-4365, 2006.

[24] Adam E Cohen and WE Moerner. Controlling brownian motion of single protein molecules and single fluorophores in aqueous buffer. Optics express, 16(10):6941-6956, 2008 .

[25] HG Craighead, SM Stavis, and KT Samiee. Nanodevices for single molecule studies. In Controlled Nanoscale Motion, pages 271-301. Springer, 2007.

[26] Nunilo Cremades, Samuel IA Cohen, Emma Deas, Andrey Y Abramov, Allen Y Chen, Angel Orte, Massimo Sandal, Richard W Clarke, Paul Dunne, Francesco A Aprile, et al. Direct observation of the interconversion of normal and toxic forms of $\alpha$-synuclein. Cell, 149(5):1048-1059, 2012.

[27] Frans C De Schryver, Tom Vosch, Mircea Cotlet, Mark Van der Auweraer, Klaus Müllen, and Johan Hofkens. Energy dissipation in multichromophoric single dendrimers. Accounts of chemical research, 38(7):514-522, 2005.

[28] Thomas Dertinger, Victor Pacheco, Iris von der Hocht, Rudolf Hartmann, Ingo Gregor, and Jörg Enderlein. Two-focus fluorescence correlation spectroscopy: A new tool for accurate and absolute diffusion measurements. ChemPhysChem, 8(3):433-443, 2007.

[29] Hendrik Deschout, Kristiaan Neyts, and Kevin Braeckmans. The influence of movement on the localization precision of sub-resolution particles in fluorescence microscopy. Journal of biophotonics, 5(1):97-109, 2012.

[30] S Devasenathipathy and JG Santiago. Electrokinetic flow diagnostics. In Microscale Diagnostic Techniques, pages 113-154. Springer, 2005. 
[31] Enzo Di Fabrizio, Sebastian Schlücker, Jérôme Wenger, Raju Regmi, Hervé Rigneault, Giuseppe Calafiore, Melanie West, Stefano Cabrini, Monika Fleischer, Niek F Van Hulst, et al. Roadmap on biosensing and photonics with advanced nano-optical methods. Journal of Optics, 18(6):063003, 2016.

[32] Chuanhua Duan, Wei Wang, and Quan Xie. Review article: Fabrication of nanofluidic devices. Biomicrofluidics, 7(2):026501, 2013.

[33] Nela Durisic, Lara Laparra-Cuervo, Ángel Sandoval-Álvarez, Joseph Steven Borbely, and Melike Lakadamyali. Single-molecule evaluation of fluorescent protein photoactivation efficiency using an in vivo nanotemplate. Nature methods, 11(2):156-162, 2014.

[34] John T Edward. Molecular volumes and the stokes-einstein equation. J. chem. Educ, 47(4):261, 1970.

[35] John Eid, Adrian Fehr, Jeremy Gray, Khai Luong, John Lyle, Geoff Otto, Paul Peluso, David Rank, Primo Baybayan, Brad Bettman, et al. Real-time dna sequencing from single polymerase molecules. Science, 323(5910):133-138, 2009.

[36] Jörg Enderlein, Ingo Gregor, Digambara Patra, Thomas Dertinger, and U Benjamin Kaupp. Performance of fluorescence correlation spectroscopy for measuring diffusion and concentration. ChemPhysChem, 6(11):2324-2336, 2005.

[37] Jörg Enderlein, David L Robbins, W Patrick Ambrose, Peter M Goodwin, and Richard A Keller. The statistics of single molecule detection: an overview. Bioimaging, 5(3):88-98, 1997.

[38] Jörg Enderlein, David L Robbins, W Patrick Ambrose, and Richard A Keller. Molecular shot noise, burst size distribution, and single-molecule detection in fluid flow: effects of multiple occupancy. The Journal of Physical Chemistry A, 102(30):6089-6094, 1998.

[39] Sanli Faez, Yoav Lahini, Stefan Weidlich, Rees F Garmann, Katrin Wondraczek, Matthias Zeisberger, Markus A Schmidt, Michel Orrit, and Vinothan N Manoharan. Fast, label-free tracking of single viruses and weakly scattering nanoparticles in a nanofluidic optical fiber. ACS nano, 9(12):12349-12357, 2015. 
[40] Alexander P Fields and Adam E Cohen. Electrokinetic trapping at the one nanometer limit. Proceedings of the National Academy of Sciences, 108(22):8937-8942, 2011.

[41] Mathieu Foquet, Jonas Korlach, Warren Zipfel, Watt W Webb, and Harold G Craighead. Dna fragment sizing by single molecule detection in submicrometer-sized closed fluidic channels. Analytical Chemistry, 74(6):1415-1422, 2002.

[42] Samantha Fore, Ted A Laurence, Christopher W Hollars, and Thomas Huser. Counting constituents in molecular complexes by fluorescence photon antibunching. IEEE Journal of Selected Topics in Quantum Electronics, 13(4):996-1005, 2007.

[43] Sarah M Friedrich, Helena C Zec, and Tza-Huei Wang. Analysis of single nucleic acid molecules in micro-and nano-fluidics. Lab on a Chip, 16(5):790-811, 2016.

[44] Lori S Goldner, Ana M Jofre, and Jianyong Tang. Droplet confinement and fluorescence measurement of single molecules. Methods in enzymology, 472:61-88, 2010.

[45] Peter M Goodwin, Mitchell E Johnson, John C Martin, W Patrick Ambrose, Babetta L Marrone, James H Jett, and Richard A Keller. Rapid sizing of individual fluorescently stained dna fragments by flow cytometry. Nucleic Acids Research, 21(4):803-806, 1993.

[46] Travis J Gould, Mudalige S Gunewardene, Manasa V Gudheti, Vladislav V Verkhusha, Shu-Rong Yin, Julie A Gosse, and Samuel T Hess. Nanoscale imaging of molecular positions and anisotropies. Nature methods, 5(12):1027-1030, 2008.

[47] Kristin S Grußmayer and Dirk-Peter Herten. Photon antibunching in single molecule fluorescence spectroscopy. In Advanced Photon Counting, pages 159-190. Springer, 2014 .

[48] L Jay Guo, Xing Cheng, and Chia-Fu Chou. Fabrication of size-controllable nanofluidic channels by nanoimprinting and its application for dna stretching. Nano letters, $4(1): 69-73,2004$.

[49] Taekjip Ha. Single-molecule fluorescence resonance energy transfer. Methods, 25(1):78$86,2001$.

[50] Taekjip Ha. Single-molecule methods leap ahead. Nature methods, 11(10):1015, 2014. 
[51] Taekjip Ha, Thilo Enderle, DF Ogletree, Daniel S Chemla, Paul R Selvin, and Shimon Weiss. Probing the interaction between two single molecules: fluorescence resonance energy transfer between a single donor and a single acceptor. Proceedings of the $\mathrm{Na}$ tional Academy of Sciences, 93(13):6264-6268, 1996.

[52] Taekjip Ha and Philip Tinnefeld. Photophysics of fluorescent probes for single-molecule biophysics and super-resolution imaging. Annual review of physical chemistry, 63:595$617,2012$.

[53] Jason J Han, Csaba Kiss, Andrew RM Bradbury, and James H Werner. Time-resolved, confocal single-molecule tracking of individual organic dyes and fluorescent proteins in three dimensions. ACS nano, 6(10):8922-8932, 2012.

[54] Michael Hirsch, Richard J Wareham, Marisa L Martin-Fernandez, Michael P Hobson, and Daniel J Rolfe. A stochastic model for electron multiplication charge-coupled devices-from theory to practice. PloS one, 8(1):e53671, 2013.

[55] Johan Hofkens, Mircea Cotlet, Tom Vosch, Philip Tinnefeld, Kenneth D Weston, Christophe Ego, Andrew Grimsdale, Klaus Müllen, David Beljonne, Jean Luc Brédas, et al. Revealing competitive förster-type resonance energy-transfer pathways in single bichromophoric molecules. Proceedings of the National Academy of Sciences, 100(23):13146-13151, 2003.

[56] Mathew H Horrocks, Laura Tosatto, Alexander J Dear, Gonzalo A Garcia, Marija Iljina, Nunilo Cremades, Mauro Dalla Serra, Tuomas PJ Knowles, Christopher M Dobson, and David Klenerman. Fast flow microfluidics and single-molecule fluorescence for the rapid characterization of $\alpha$-synuclein oligomers. Analytical chemistry, 87(17):8818-8826, 2015.

[57] Gerhard Hummer, Franziska Fricke, and Mike Heilemann. Model-independent counting of molecules in single-molecule localization microscopy. Molecular Biology of the Cell, 27(22):3637-3644, 2016.

[58] Marija Iljina, Gonzalo A Garcia, Mathew H Horrocks, Laura Tosatto, Minee L Choi, Kristina A Ganzinger, Andrey Y Abramov, Sonia Gandhi, Nicholas W Wood, Nunilo 
Cremades, et al. Kinetic model of the aggregation of alpha-synuclein provides insights into prion-like spreading. Proceedings of the National Academy of Sciences, 113(9):E1206-E1215, 2016.

[59] Ralf Jungmann, Maier S Avendaño, Mingjie Dai, Johannes B Woehrstein, Sarit S Agasti, Zachary Feiger, Avital Rodal, and Peng Yin. Quantitative super-resolution imaging with qpaint. Nature methods, 2016.

[60] Ralf Jungmann, Christian Steinhauer, Max Scheible, Anton Kuzyk, Philip Tinnefeld, and Friedrich C Simmel. Single-molecule kinetics and super-resolution microscopy by fluorescence imaging of transient binding on dna origami. Nano letters, 10(11):47564761, 2010.

[61] Peter Kapusta, Michael Wahl, Aleš Benda, Martin Hof, and Jörg Enderlein. Fluorescence lifetime correlation spectroscopy. Journal of Fluorescence, 17(1):43-48, 2007.

[62] Peet Kask, Kaupo Palo, Dirk Ullmann, and Karsten Gall. Fluorescence-intensity distribution analysis and its application in biomolecular detection technology. Proceedings of the National Academy of Sciences, 96(24):13756-13761, 1999.

[63] Philipp J Keller, Annette D Schmidt, Joachim Wittbrodt, and Ernst HK Stelzer. Reconstruction of zebrafish early embryonic development by scanned light sheet microscopy. science, 322(5904):1065-1069, 2008.

[64] H Jeff Kimble, Mario Dagenais, and Leonard Mandel. Photon antibunching in resonance fluorescence. Physical Review Letters, 39(11):691, 1977.

[65] HJ Kimble and L Mandel. Theory of resonance fluorescence. Physical Review A, 13(6):2123, 1976 .

[66] Christian Kurtsiefer, Sonja Mayer, Patrick Zarda, and Harald Weinfurter. Stable solidstate source of single photons. Physical review letters, 85(2):290, 2000.

[67] Joseph R Lakowicz. Time-domain lifetime measurements. In Principles of fluorescence spectroscopy, pages 95-140. Springer, 1999. 
[68] Sang-Hyuk Lee, Jae Yen Shin, Antony Lee, and Carlos Bustamante. Counting single photoactivatable fluorescent molecules by photoactivated localization microscopy (palm). Proceedings of the National Academy of Sciences, 109(43):17436-17441, 2012.

[69] Sabrina R Leslie, Alexander P Fields, and Adam E Cohen. Convex lens-induced confinement for imaging single molecules. Analytical chemistry, 82(14):6224-6229, 2010.

[70] John F Lesoine, Prahnesh A Venkataraman, Peter C Maloney, Mark E Dumont, and Lukas Novotny. Nanochannel-based single molecule recycling. Nano letters, 12(6):3273-3278, 2012.

[71] Michael J Levene, Jonas Korlach, Stephen W Turner, Mathieu Foquet, Harold G Craighead, and Watt W Webb. Zero-mode waveguides for single-molecule analysis at high concentrations. Science, 299(5607):682-686, 2003.

[72] Kelvin J Liu, Malcolm V Brock, Ie-Ming Shih, and Tza-Huei Wang. Decoding circulating nucleic acids in human serum using microfluidic single molecule spectroscopy. Journal of the American Chemical Society, 132(16):5793-5798, 2010.

[73] Kelvin J Liu, Tushar D Rane, Yi Zhang, and Tza-Huei Wang. Single-molecule analysis enables free solution hydrodynamic separation using yoctomole levels of dna. Journal of the American Chemical Society, 133(18):6898-6901, 2011.

[74] Kelvin J Liu and Tza-Huei Wang. Cylindrical illumination confocal spectroscopy: rectifying the limitations of confocal single molecule spectroscopy through onedimensional beam shaping. Biophysical journal, 95(6):2964-2975, 2008.

[75] Samuel J Lord, Hsiao-lu D Lee, and WE Moerner. Single-molecule spectroscopy and imaging of biomolecules in living cells. Analytical chemistry, 82(6):2192-2203, 2010.

[76] Anna Löschberger, Sebastian van de Linde, Marie-Christine Dabauvalle, Bernd Rieger, Mike Heilemann, Georg Krohne, and Markus Sauer. Super-resolution imaging visualizes the eightfold symmetry of gp210 proteins around the nuclear pore complex and resolves the central channel with nanometer resolution. J Cell Sci, 125(3):570-575, 2012. 
[77] Anna B Loveland, Satoshi Habuchi, Johannes C Walter, and Antoine M Van Oijen. A general approach to break the concentration barrier in single-molecule imaging. Nature methods, 9(10):987-992, 2012.

[78] Sonny Ly, Jitka Petrlova, Thomas Huser, Samantha Fore, Tingjuan Gao, John Voss, and Ted A Laurence. Stoichiometry of reconstituted high-density lipoproteins in the hydrated state determined by photon antibunching. Biophysical journal, 101(4):970975, 2011.

[79] Nicholas P Machara, Peter M Goodwin, Jörg Enderlein, David J Semin, and Richard A Keller. Efficient detection of single molecules eluting off an optically trapped microsphere. Bioimaging, 6(1):33-42, 1998.

[80] Michael Maus, Mircea Cotlet, Johan Hofkens, Thomas Gensch, Frans C De Schryver, J Schaffer, and CAM Seidel. An experimental comparison of the maximum likelihood estimation and nonlinear least-squares fluorescence lifetime analysis of single molecules. Analytical chemistry, 73(9):2078-2086, 2001.

[81] P Michler, A Imamoğlu, MD Mason, PJ Carson, GF Strouse, and SK Buratto. Quantum correlation among photons from a single quantum dot at room temperature. $\mathrm{Na}$ ture, 406(6799):968-970, 2000.

[82] D Mijatovic, JCT Eijkel, and A Van Den Berg. Technologies for nanofluidic systems: top down vs. bottom up - a review. Lab on a Chip, 5(5):492-500, 2005.

[83] WE Moerner and Michel Orrit. Illuminating single molecules in condensed matter. Science, 283(5408):1670-1676, 1999.

[84] William Esco Moerner and Lothar Kador. Optical detection and spectroscopy of single molecules in a solid. Physical review letters, 62(21):2535, 1989.

[85] Barbara K Müller, Evgeny Zaychikov, Christoph Bräuchle, and Don C Lamb. Pulsed interleaved excitation. Biophysical journal, 89(5):3508-3522, 2005.

[86] M Napoli, JCT Eijkel, and S Pennathur. Nanofluidic technology for biomolecule applications: a critical review. Lab on a Chip, 10(8):957-985, 2010. 
[87] Burak Okumus, Timothy J Wilson, David MJ Lilley, and Taekjip Ha. Vesicle encapsulation studies reveal that single molecule ribozyme heterogeneities are intrinsic. Biophysical journal, 87(4):2798-2806, 2004.

[88] Michel Orrit and J Bernard. Single pentacene molecules detected by fluorescence excitation in a p-terphenyl crystal. Physical review letters, 65(21):2716, 1990.

[89] George Patterson, Michael Davidson, Suliana Manley, and Jennifer LippincottSchwartz. Superresolution imaging using single-molecule localization. Annual review of physical chemistry, 61:345-367, 2010.

[90] Jeppe T Pedersen and Niels HH Heegaard. Analysis of protein aggregation in neurodegenerative disease, 2013.

[91] Christoph M Pieper and Jörg Enderlein. Fluorescence correlation spectroscopy as a tool for measuring the rotational diffusion of macromolecules. Chemical Physics Letters, 516(1):1-11, 2011.

[92] Hong Qian, Michael P Sheetz, and Elliot L Elson. Single particle tracking. analysis of diffusion and flow in two-dimensional systems. Biophysical journal, 60(4):910-921, 1991.

[93] Walter Reisner, Niels B Larsen, Asli Silahtaroglu, Anders Kristensen, Niels Tommerup, Jonas O Tegenfeldt, and Henrik Flyvbjerg. Single-molecule denaturation mapping of dna in nanofluidic channels. Proceedings of the National Academy of Sciences, 107(30):13294-13299, 2010.

[94] Walter Reisner, Keith J Morton, Robert Riehn, Yan Mei Wang, Zhaoning Yu, Michael Rosen, James C Sturm, Stephen Y Chou, Erwin Frey, and Robert H Austin. Statics and dynamics of single dna molecules confined in nanochannels. Physical Review Letters, 94(19):196101, 2005.

[95] Andrew Robinson and Antoine M Van Oijen. Bacterial replication, transcription and translation: mechanistic insights from single-molecule biochemical studies. Nature Reviews Microbiology, 11(5):303-315, 2013. 
[96] Geoffrey C Rollins, Jae Yen Shin, Carlos Bustamante, and Steve Pressé. Stochastic approach to the molecular counting problem in superresolution microscopy. Proceedings of the National Academy of Sciences, 112(2):E110-E118, 2015.

[97] Christopher A Ross and Michelle A Poirier. Protein aggregation and neurodegenerative disease. Nature Medicine, 10(7):S10, 2004.

[98] Christopher A Ross and Michelle A Poirier. What is the role of protein aggregation in neurodegeneration? Nature reviews Molecular cell biology, 6(11):891-898, 2005.

[99] Michael J Rust, Mark Bates, and Xiaowei Zhuang. Sub-diffraction-limit imaging by stochastic optical reconstruction microscopy (storm). Nature methods, 3(10):793-796, 2006.

[100] Steffen J Sahl and WE Moerner. Super-resolution fluorescence imaging with single molecules. Current opinion in structural biology, 23(5):778-787, 2013.

[101] Markus Sauer, Johan Hofkens, and Jörg Enderlein. Handbook of fluorescence spectroscopy and imaging: from ensemble to single molecules. John Wiley \& Sons, 2010.

[102] J Schuster, F Cichos, and C von Borczyskowski. Diffusion measurements by singlemolecule spot-size analysis. The Journal of Physical Chemistry A, 106(22):5403-5406, 2002 .

[103] LI Segerink and JCT Eijkel. Nanofluidics in point of care applications. Lab on a Chip, 14(17):3201-3205, 2014.

[104] Sarah L Shammas, Gonzalo A Garcia, Satish Kumar, Magnus Kjaergaard, Mathew H Horrocks, Nadia Shivji, Eva Mandelkow, Tuomas PJ Knowles, Eckhard Mandelkow, and David Klenerman. A mechanistic model of tau amyloid aggregation based on direct observation of oligomers. Nature communications, 6, 2015.

[105] Min Ju Shon and Adam E Cohen. Mass action at the single-molecule level. Journal of the American Chemical Society, 134(35):14618-14623, 2012. 
[106] Evangelos Sisamakis, Alessandro Valeri, Stanislav Kalinin, Paul J Rothwell, and Claus AM Seidel. Accurate single-molecule fret studies using multiparameter fluorescence detection. Methods in enzymology, 475:455-514, 2010.

[107] Simon Christoph Stein and Jan Thiart. Trackntrace: A simple and extendable opensource framework for developing single-molecule localization and tracking algorithms. Scientific Reports, 6, 2016.

[108] Ernst HK Stelzer. Light-sheet fluorescence microscopy for quantitative biology. Nature methods, 12(1):23-28, 2015.

[109] Jan Sykora, Karin Kaiser, Ingo Gregor, Wolfgang Bönigk, Günther Schmalzing, and Jörg Enderlein. Exploring fluorescence antibunching in solution to determine the stoichiometry of molecular complexes. Analytical chemistry, 79(11):4040-4049, 2007.

[110] Haisen Ta, Jan Keller, Markus Haltmeier, Sinem K Saka, Jürgen Schmied, Felipe Opazo, Philip Tinnefeld, Axel Munk, and Stefan W Hell. Mapping molecules in scanning far-field fluorescence nanoscopy. Nature communications, 6, 2015.

[111] Haisen Ta, Alexander Kiel, Michael Wahl, and Dirk-Peter Herten. Experimental approach to extend the range for counting fluorescent molecules based on photonantibunching. Physical Chemistry Chemical Physics, 12(35):10295-10300, 2010.

[112] Abel L Thangawng, Melody A Swartz, Matthew R Glucksberg, and Rodney S Ruoff. Bond-detach lithography: a method for micro/nanolithography by precision pdms patterning. small, 3(1):132-138, 2007.

[113] Alexander Thiessen, Jan Vogelsang, Takuji Adachi, Florian Steiner, David Vanden Bout, and John M Lupton. Unraveling the chromophoric disorder of poly (3hexylthiophene). Proceedings of the National Academy of Sciences, 110(38):E3550E3556, 2013.

[114] Makio Tokunaga, Kazuo Kitamura, Kiwamu Saito, Atsuko Hikikoshi Iwane, and Toshio Yanagida. Single molecule imaging of fluorophores and enzymatic reactions achieved by objective-type total internal reflection fluorescence microscopy. Biochemical and biophysical research communications, 235(1):47-53, 1997. 
[115] Swati Tyagi, Virginia VanDelinder, Niccolò Banterle, Gustavo Fuertes, Sigrid Milles, Morgane Agez, and Edward A Lemke. Continuous throughput and long-term observation of single-molecule fret without immobilization. Nature methods, 11(3):297-300, 2014 .

[116] Maximilian H Ulbrich and Ehud Y Isacoff. Subunit counting in membrane-bound proteins. Nature methods, 4(4):319-321, 2007.

[117] Quan Wang. Enabling multivariate investigation of single-molecule dynamics in solution by counteracting Brownian motion. PhD thesis, Stanford University, 2016.

[118] Quan Wang and WE Moerner. Lifetime and spectrally resolved characterization of the photodynamics of single fluorophores in solution using the anti-brownian electrokinetic trap. The Journal of Physical Chemistry B, 117(16):4641-4648, 2012.

[119] Kenneth D Weston, Martina Dyck, Philip Tinnefeld, Christian Müller, Dirk P Herten, and Markus Sauer. Measuring the number of independent emitters in single-molecule fluorescence images and trajectories using coincident photons. Analytical chemistry, 74(20):5342-5349, 2002.

[120] Jerker Widengren, Ulo Mets, Rudolf Rigler, et al. Fluorescence correlation spectroscopy of triplet-states in solution-a theoretical and experimental-study. Journal of Physical Chemistry, 99(36):13368-13379, 1995.

[121] Hongkai Wu, Bo Huang, and Richard N Zare. Construction of microfluidic chips using polydimethylsiloxane for adhesive bonding. Lab on a Chip, 5(12):1393-1398, 2005.

[122] Toshio Yanagida and Yoshiharu Ishii. Single molecule dynamics in life science. John Wiley \& Sons, 2008.

[123] Haw Yang, Guobin Luo, Pallop Karnchanaphanurach, Tai-Man Louie, Ivan Rech, Sergio Cova, Luying Xun, and X Sunney Xie. Protein conformational dynamics probed by single-molecule electron transfer. Science, 302(5643):262-266, 2003.

[124] Ahmet Yildiz, Joseph N Forkey, Sean A McKinney, Taekjip Ha, Yale E Goldman, and Paul R Selvin. Myosin v walks hand-over-hand: single fluorophore imaging with 1.5-nm localization. science, 300(5628):2061-2065, 2003. 
[125] Shannon Kian Zareh, Michael C DeSantis, Jonathan M Kessler, Je-Luen Li, and YM Wang. Single-image diffusion coefficient measurements of proteins in free solution. Biophysical journal, 102(7):1685-1691, 2012.

[126] Zhengyang Zhang, Samuel J Kenny, Margaret Hauser, Wan Li, and Ke Xu. Ultrahighthroughput single-molecule spectroscopy and spectrally resolved super-resolution microscopy. Nature methods, 12(10):935-938, 2015.

[127] Yanhui Zhao, Danqi Chen, Hongjun Yue, Jarrod B French, Joseph Rufo, Stephen J Benkovic, and Tony Jun Huang. Lab-on-a-chip technologies for single-molecule studies. Lab on a Chip, 13(12):2183-2198, 2013.

[128] Niels Zijlstra, Christian Blum, Ine MJ Segers-Nolten, Mireille MAE Claessens, and Vinod Subramaniam. Molecular composition of sub-stoichiometrically labeled $\alpha$-synuclein oligomers determined by single-molecule photobleaching. Angewandte Chemie International Edition, 51(35):8821-8824, 2012. 


\section{Acronyms}

smFRET single-molecule Foerster Resonance Energy Transfer

TIRF total internal reflection fluorescence

PD Parkinson's disease

FCS Fluorescence Correlation Spectroscopy

SNR signal to noise ratio

PCH photon-counting histogram

ROI region of interest

FIDA Fluorescence Intensity Distribution Analysis

MDF molecular detection function

BSD burst size distribution

ABEL Anti-Brownian Electrokinetic

PSF Point Spread Function

ZMWs zero-mode waveguides

ACF Autocorrelation Function

dsDNA double-stranded Deoxyribonucleic acid

SPT Single-particle tracking 


\section{Acknowledgments}

First of all, I would like to thank my supervisor, Prof. Jörg Enderlein, for his continuous support of this project and my study, for offering me the opportunities to take a multitude of projects, for his patience and trust on me. His guidance helped me in all my time in the group and writing of this thesis.

I would like to thank the members of my thesis committee: Prof. Helmut Grubmüller and Prof. Fred Wouters, not only for taking the time to regularly monitor the progress of my work, but also for their advice and encouragement.

I would like to thank Dr. Ingo Gregor for his constantly providing me precious instructions with his immense knowledge. I would like to thank my great office mates, former and present, Anja, Dirk, Roman, Steffen, for the wonderful office atmosphere and the great time we shared. Special thanks go to Simon, for his generous contribution to this project and inspiring discussions. I also thank Jan for sharing his apartment with me for three months. My sincere thanks also go to Qui, Olaf, Mira, Kerstin, Phillip, Narain, Man, Weixing, Daja, Alexey, Anna, Sid, Stefanie, Melanie, for all the help I received when I was new in the group. I also thank all the other group members, and wish you all the best for your future.

Many thanks go to the secretaries and workshop staff of the institute. I also would like to thank the PBCS office for the great support.

I am extremely grateful to all the discussions and suggestions from Dr. Haisen Ta and Dr. Say Hwa Tan, helping me solve so many problems in this project.

I acknowledge the financial support of this work by SFB 755 and SFB 803 (DFG).

Lastly, I am grateful to my parents for their unfailing support throughout my years of study. 